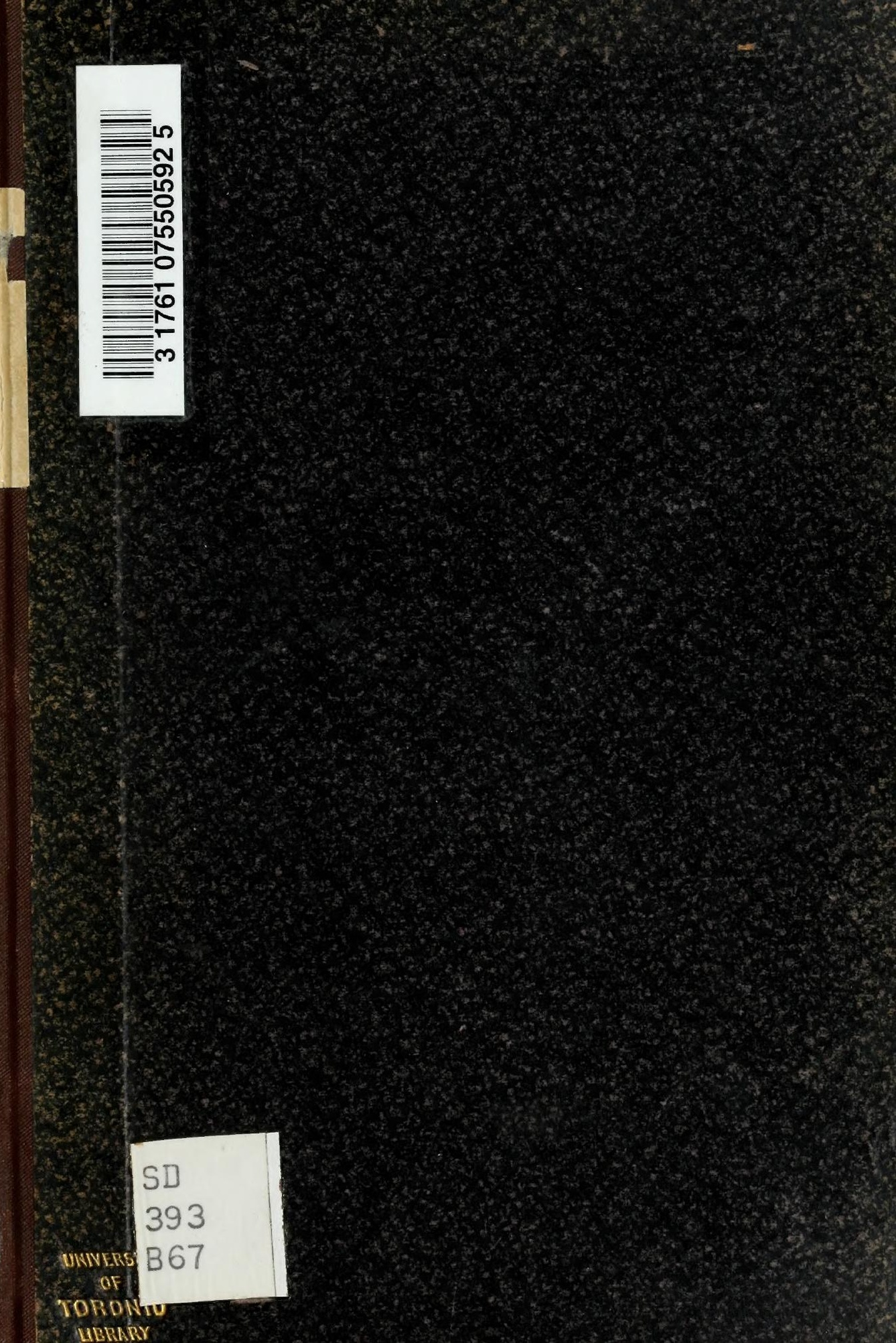






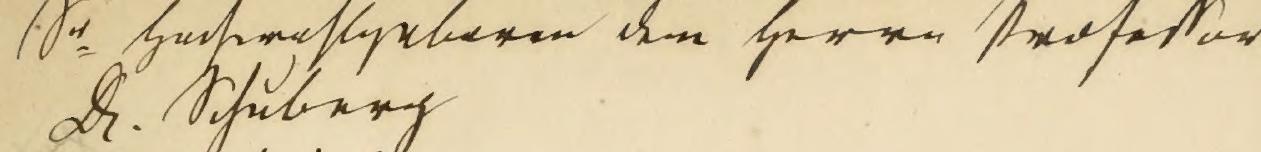
gaopustimponde man

\section{Das foritliche}

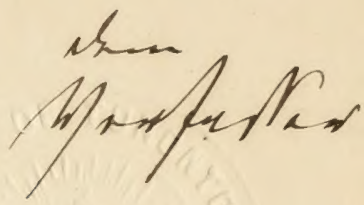

Weiferprozent

vort

Geinrich $\mathfrak{L}$ idowig Boje,

Grob̧hergoglid heffifhem oberforftoireftor i. $p$.

\section{LIBRARY}

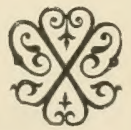

UNIVERSITY OF TORONTO

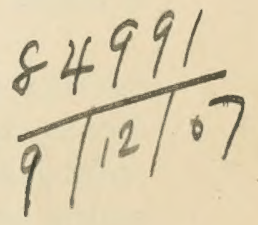

פerlin.

Derlag von $\mathfrak{P} a \mathfrak{l} \mathfrak{P} \mathfrak{a} \mathfrak{e}$. 1889. 


$$
\begin{aligned}
& 50 \\
& 393 \\
& B 67
\end{aligned}
$$




\section{$\mathfrak{d} \circ \mathfrak{r} \mathfrak{w} \circ \mathfrak{r} \mathfrak{f}$}

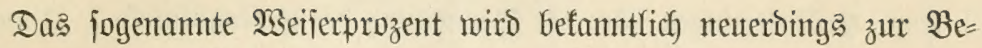
gründung Der forjtfichen Finanzrectunung beionders herborgeboben. Da Dasfelbe jeonch in Der Weife, wie es von den Bodenreinerträglern bargeftellt wird, weiter nichts ijt, als eine in algebraijche formeln

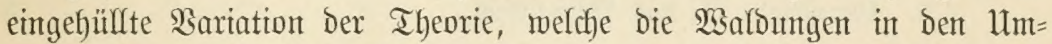
triebszeiten Der Göchiten Bobenermartungsmerte bemirtjchaftet haben will, und ba Durdf dasjelbe ber wabre Sactjerbalt biejer Iheorie verjaleiert wiro, jo habe ich verjucht, genanntes $\mathfrak{B e i f e r p r o z e n t ~ i n ~ b e n ~ n a c h j t e f e n d e n ~}$ Blättern näher żu erörtern und auf jeine praftijche S(nmenobarfeit zu prüfen.

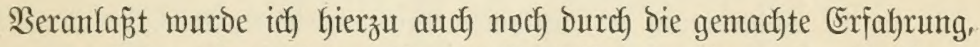
Dá̉ namentlich) viele unierer älteren Praftifer Durchaus im Infflaren.

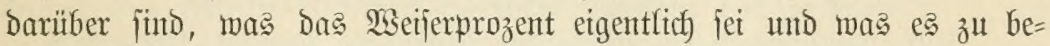
Deuten habe.

Recht gut jehe ich ein, bar, meine Darjtellung, welche bie gänzliche Unbrauchbarfeit Der Bobenreinertrag = und $\mathfrak{B}$ etjerprozent= Theorie zur

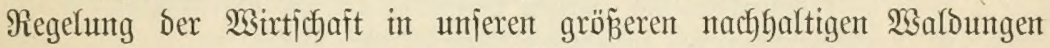

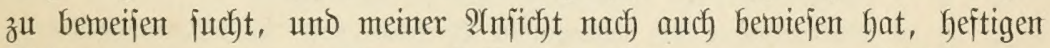

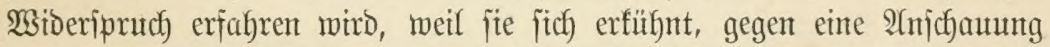
Front zu machen, welche nun fchon über 30 Jabre lang zur förmlichent Miodejache geworben ift und bie forjtliche sitteratur fajt volljtändig be=

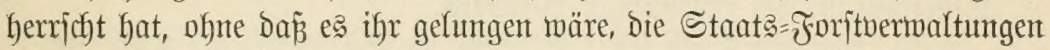

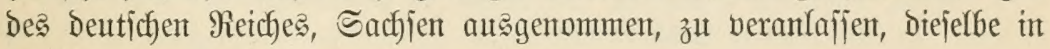
bie \$raxis überzuführen.

Auch in Den Staatsmaldungen des Röntgreichs Sachjen foll bieje

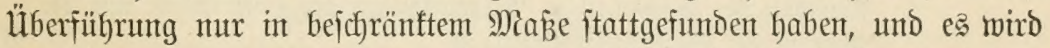

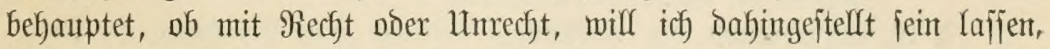

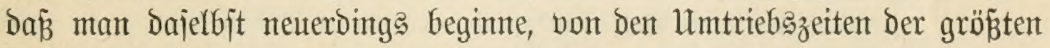
Bodenerwartungsmerte mieber abz̧ugehent.

Darmjtadt, Den 31. Dftober 1889. 


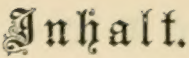

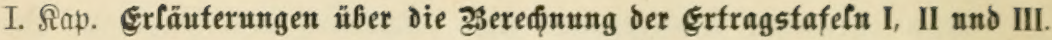

§ 1. AIgemeine Borbemerkng ....................... Seite

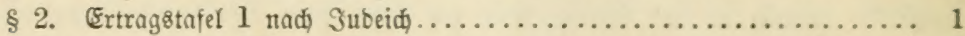

§ 3. Ertragstafel II nach pöpel $\ldots \ldots \ldots \ldots \ldots \ldots \ldots \ldots \ldots \ldots \ldots$

§ 4. Ertragstafel III nad, Sdimappad)....................... 5

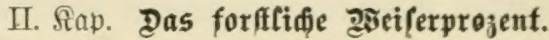

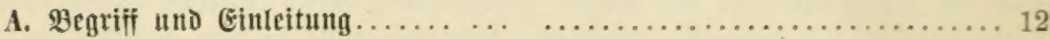

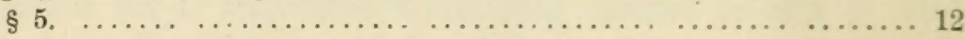

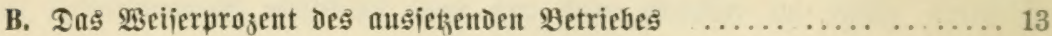

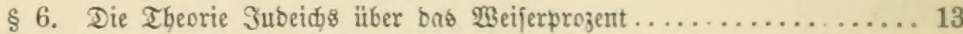

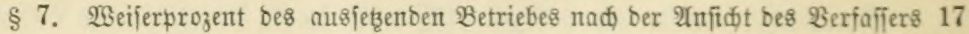

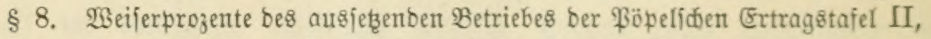
nad) ber oben erwäbnten Subeidjdent frormel II beredinet ........ 19

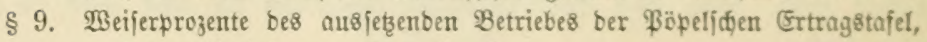
nad ber Metbobe von Sraft berednet .. .............. 19

8 10. Weiferprozente bes auళjetzenben Betriebes nad (5uftav நeber....... 21

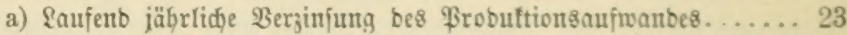

b) Durdjidnittlid jäbrlidbe Werzinjung beళ Brobuftionsaufmanbe8 . 27

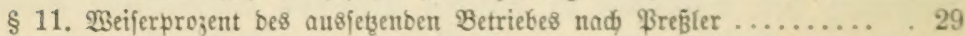

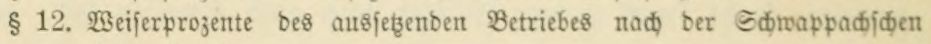

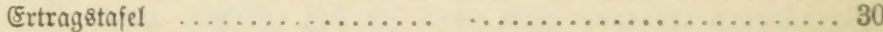

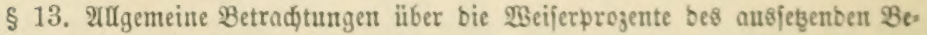
triebes ................................. 30

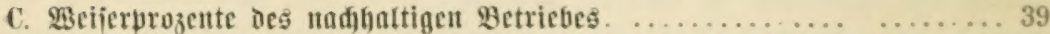

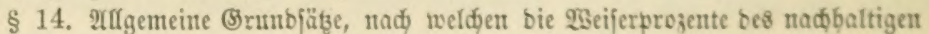
Betriebes ju berednen fint ... .. .................... 39

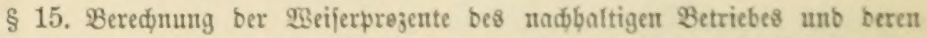

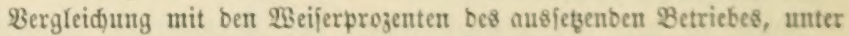
2nnabme ber gegenwärtigen (5ebraudsmerte ber ఏoljbefänbe unb ber Marima ber Bobenerwartungswerte . ............. 47

\$ 16. 23efjerprozente be\& naфbaltigen Betriebes unter Inventung ber Be* braudbstwerte ber Đolabeftänbe unb verídicbener Bobemwerte ....... 52

\$ 17. 23eiferprozente be8 nadbaltigen 2 etriebes unter Inmenbung ber mut-

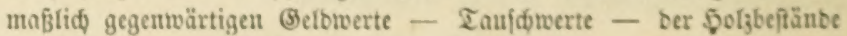

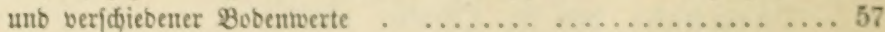

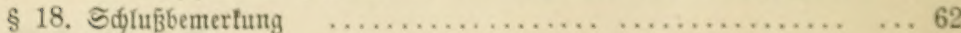




\section{Erjtes Kapitel.

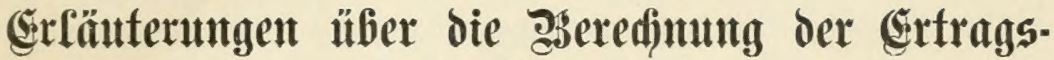 tafefn I, II anto III.}

\section{$\S 1$. \\ Allgenteite Borbenterfutg.}

Um Die Tragmeite und praftijche 2 (ntmentbarfeit ber vielen in ber foritlichent Statif eingefübrten algebraijchen Formeln beurteilen zu fömen, ijt es meiner S(mjicht nach) Durchaus nötig, biejelfen an Der Natur ent= nommenen Bablenbeippielen zut prüfen unt zut erörtern. Mant wirb bant

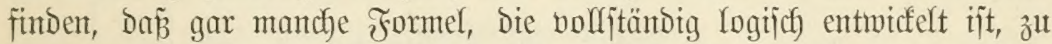

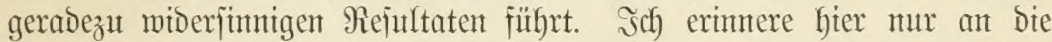
(3). Scenerjue Formel über Den Internebmergeminn, weldfent icf) im Februarhejte Des $:$ Baurjchen Centralblattes von 1889, S. 73 2., auts=

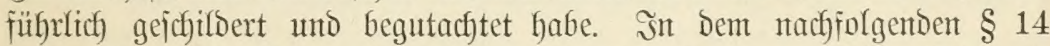
habe ich Die (5. Seyerjche Theorie hierüber furz entwidfelt. Die ver= jefiedenen über Das jogenannte 2 Seijerprozent aufgejtellten Theorieen habe ich) in ben nachjolgenden Blättern nicht alfein itreng mathematijch ent= widfelt, jonbern aud Durch biele, recht müfjame Berechuntigen erforbernde Tabellen anjchaulidy zut machen gejutht, weldye ich an bie am Echlujie Diejes Sapitels abgeonuteften 3 Ertragstafeln angefnilpft hahe, beren $3 \mathfrak{u}=$ jammenitelfung und Berect)mung vor alfem einer genauen Erörterung bedari.

\section{$\S 2$. \\ (Ertragstajel I ttad) Ittbeidy.}

Dieje Tafel, weldye Die Erträge eines 1 ha gropien Fichtenbejtands

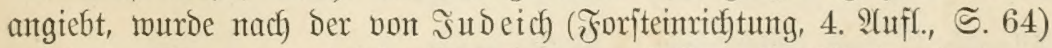
gegebenten Sertertragstafel teils zujammengeitellt uno teils berechnet.

Die Spalten a, b und c fint ber Subeidfichen Iafel entnommen.

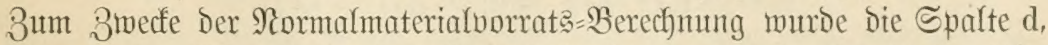
Summe der Alltersftufen des şauptbeitands, bon mir eingefügt. Die= 


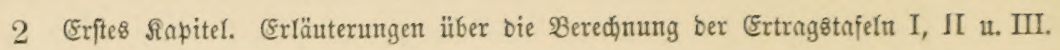

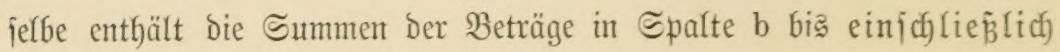
ber in Spalte a eingetragenen $5 \mathfrak{c}$ olzalter.

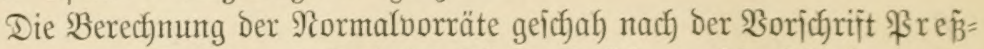

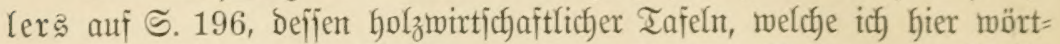
lich) wiebergeben will. Man vergleiche aud meine Beiträge zur $\mathfrak{W a l d}=$

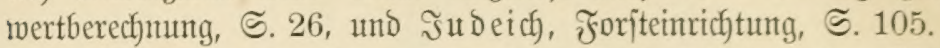

"Went man in einer von $\mathrm{n}$ zu $\mathrm{n}$ Jabren ipringenden (Ertragstafel

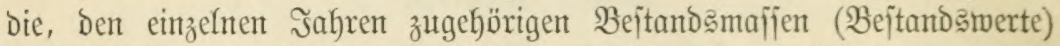

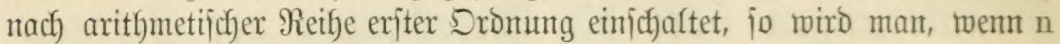
nicht zu groß́, etwa $=10$ Jahre ijt, Der Wabrbeit mentig zu nabe. treten, und in biejem Falle hat man nach Den (Bejetzen Der aritfmetijchen Reiben:

\begin{tabular}{|c|c|c|c|c|c|}
\hline 2ulter & Mafīen & & & Maijen ('WBerte) & 2) ber थIter\&ftufen \\
\hline 0 & 0 & bon 0 & & 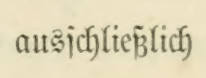 & $a=(0+a) \frac{n+1}{2}-a$ \\
\hline $\mathrm{n}$ & $a$ & i a & $"$ & " & $b=(a+b) \frac{n+1}{2}-b$ \\
\hline $2 n$ & b & $" \mathrm{~b}$ & " & $"$ & $c=(b+c) \frac{n+1}{2}-c$ \\
\hline $3 n$ & $\mathrm{c}$ & $" \quad \mathrm{c}$ & biz & émj̣chliểeliç) & $\mathrm{d}=(\mathrm{c}+\mathrm{d}) \frac{\mathrm{n}+1}{2}-0$ \\
\hline $4 n$ & d & & & & \\
\hline
\end{tabular}

$$
\begin{gathered}
\text { Summe } \begin{array}{c}
\frac{n+1}{2}(2 a+2 b+2 c+d)-(a+b+c)=N V \\
=(n+1)\left(a+b+c+\frac{d}{2}\right)-(a+b+c)=N V \\
n\left(a+b+c+\frac{d}{2}\right)+\frac{d}{2}=N V
\end{array}
\end{gathered}
$$

Dieje Formel enthält mithin ben ફ̧olzgehalt bes ältejten Jabres=

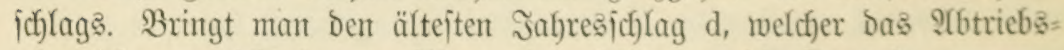
alter fdyon erreidyt hat, in $\mathfrak{A f b}_{3} \mathrm{~g}$, jo erfält man bie frormel:

$$
\mathrm{NV}=\mathrm{n}\left(\mathrm{a}+\mathrm{b}+\mathrm{c}+\frac{\mathrm{d}}{2}\right)-\frac{\mathrm{d}}{2} \text {, }
$$

uno Durch bieje Den ridfigen Normalmaterialvorrat im Frübjabre nad) Dem Stbtriebe Des ältejten Sahreşichlaģ. Scheidet man bas in ber Flammer fitebenoe $\frac{d}{2}$ aus biejer aus, jo erbält man:

$$
\mathrm{NV}=\mathrm{n}(\mathrm{a}+\mathrm{b}+\mathrm{c})+\mathrm{d}\left(\frac{\mathrm{n}-1}{2}\right),
$$

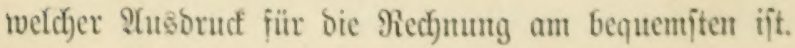




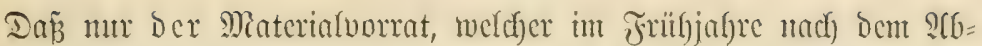

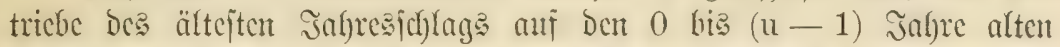

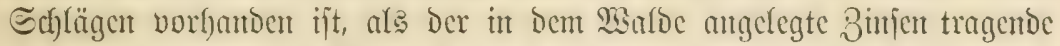

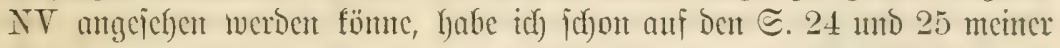

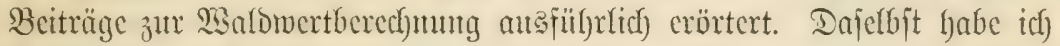
ธ. 25 folgendes gejagt:

"Ein วิl $4 \%$ ausgelichenes Sapital von 100 fl. wäd)it im Sauje Des Jaf)res zu 104 fl. an, und wiro am Ende bes Jafres burd) Zalflung Der Bimjen bon jeiten bes ๔efuldoners mieder auf ben urjprïnglidfen @tand

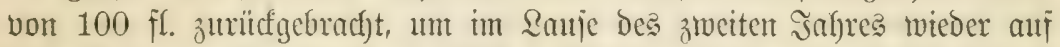

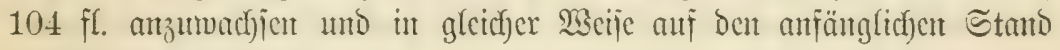
vermintoert 孔ैu werden.

E⿱ Das jimjentragende fiapital nur 100 fl. und nicht 104 fl. betrage. Sianz ১as nämliche Serbältnis findet aber bei bom Miaterialvorrate ftatt. Das Minimum Desjelben vermel)rt fich) vom Friifjabr bis zum Sgerbjt um ben

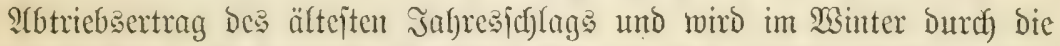
Binşahlung, D. h. Durd) bie Ernte Der auf Dem älteften Sdjlage vor=

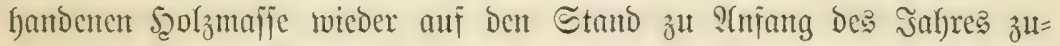

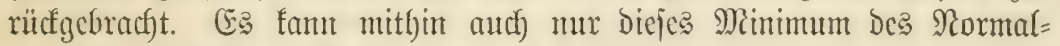
vorrats als bas im Malde angelegte Jolzfaptal antgejelen werden."

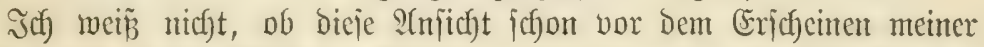
Beiträge im Jafre 1863 zur Bseltung gefommen ijt. J̃n Den neueren Sdyriften ïber $\mathfrak{W a l b w e r t b e r e c f m u n g ~ w i r d ~ b i e j e l b e ~ j e b o c h ~ a l f g e m e i n ~ a l ? ~}$ bie ricftige angenommen.

Da it Der Jubeidffden Tafel bie Merte bes 5̧auptbeitands erit mit Dem 15. Jafje beginten, io fabe id) Dem entjpredjent bei Berectumng Der NV aud) Dic jüngeren S(ltersflajjen umberïcfichtigt gelaffen, nament=

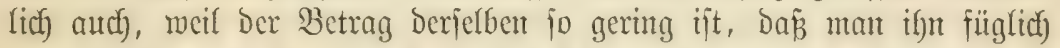
vernachläfïigen barf. Ltm 3. B. Den NV eimer normalen Betricbaflaffe von 100 ha, tweldje in Der llmtricbeszeit von 100 Jaljen belvirtidjaftet mird, zu berechnen, verfäbrt man folgendermaß̄en:

Der bei Dem Sllter 100 j̈tehende Mert Des Jeauptbejtands in Spalte b $=3780$ entipridgt Dem Bucfitaben d in umperer Formel umb bie in Spalte d bet bem :(fter 95 jtehende Summe Der Iftersftujen vom 15. biz

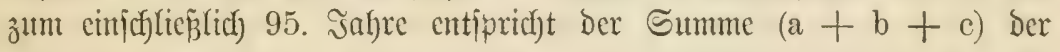
Formel. Scicrnad) ijt, ba $n=5, N V=5 \times 18829,6+3780\left(\frac{5-1}{2}\right)$ $=101708,0$. Dicjer Betrag entipridjt Dem NV ciner normalen Betriebs= Elafie von 100 ha. 


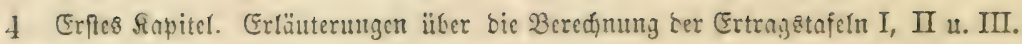

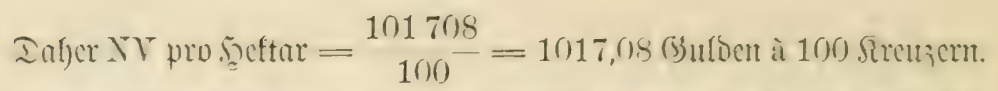

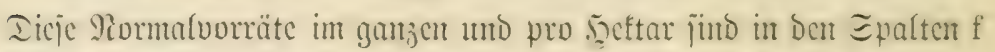
unto $g$ eingetragen.

Ia bie Jubeidfidfe Iajel bic nadffaltigen 2ialdorträge ber nor=

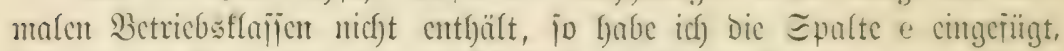

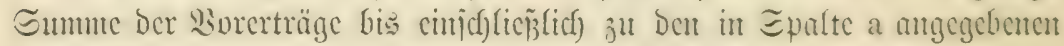
5ुolzaltern.

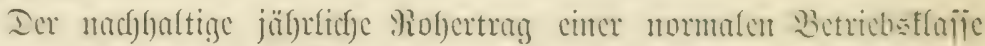

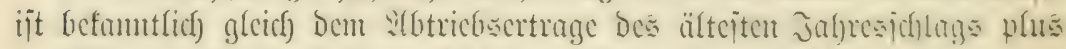

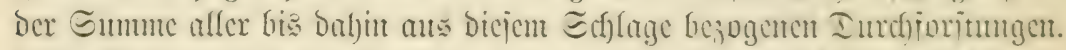
Dicje Beträge finto in Epalte he cingetragen. Ier nadjbaltige Piofertmag

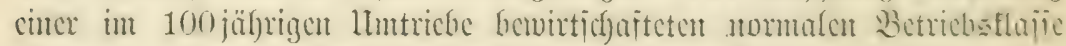
ijt mithin:

$$
\begin{aligned}
& \text { 9Lbtricbsertrag Des S(f)lagę . . . . = }=3780,0
\end{aligned}
$$

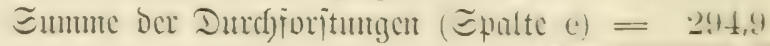

$$
\begin{aligned}
& \text { Summe }=4074,9
\end{aligned}
$$

Daber pro neftar ber ganter silaijc $=\frac{4074,9}{100}=40,75$, welde Beträge in Spalte i cingetragen fino.

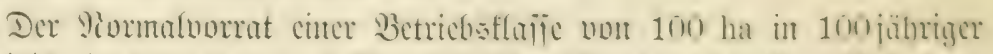

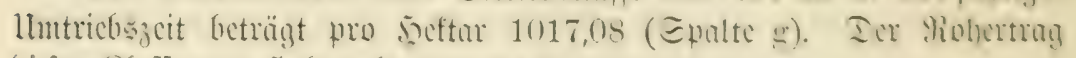
bicjer Silafic pro Şeftar Geträgt 40,75 (ङpalte i).

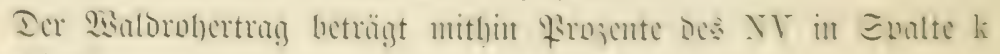
$\frac{100 \cdot 40,75}{1017,08}=4 \%$ (abgerumoct).

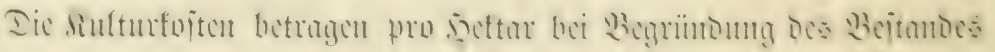

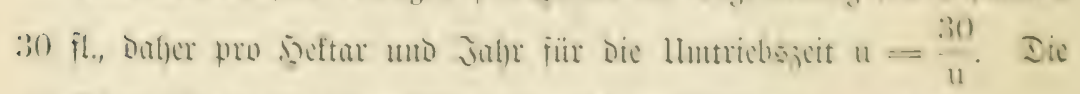

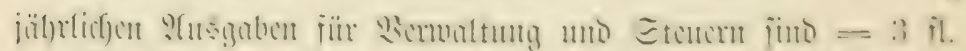

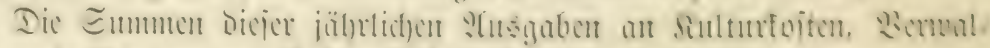

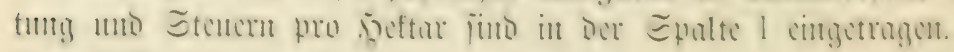

3. 2. วïr $u=100$

$$
\begin{aligned}
& \text { jüfrliç)e Siulturfojten }=\frac{30}{100}=0,30 \text { il., }
\end{aligned}
$$

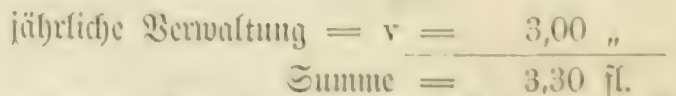

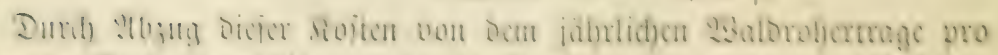

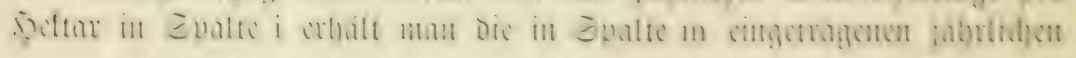




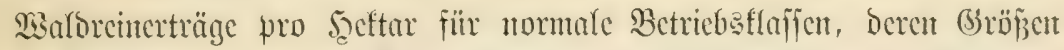
it 5eftaren unto Deren IImtricbsjeiten Den in Epalte a cingetragenen

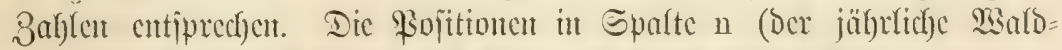

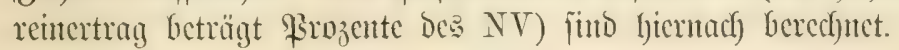

3. $\mathfrak{B}$. für $\mathrm{u}=100$

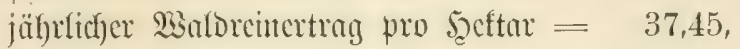

NV pro Sceftar . . . . . . $=1017,08$,

Dafer Prozent

$$
\frac{37,45 \times 100}{1017,08}=3,68 \mathrm{pCt} \text {. }
$$

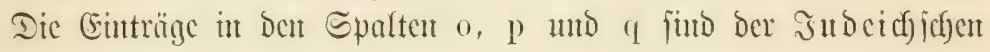
Tafel entnomment.

$$
\S 3 .
$$

\section{Ertragstajel II uad) Pöpel.}

Dic (Ertragstafel, nach) welder Tafel II fouftutert morden iit, murde

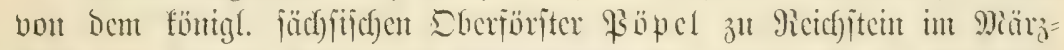

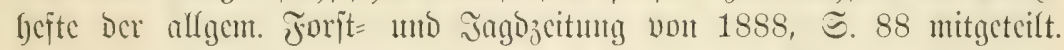
Dic Satumalerträge Deriefloen folfen nad) Den neuciten Bujanmenjtellumgen

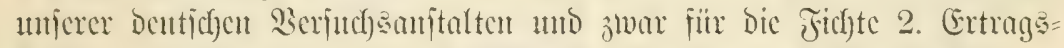

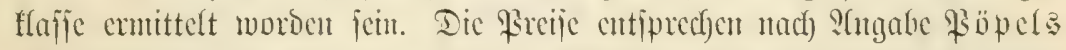
ben bortigen feit mefberen Jnferen ïblicfen. Dic anlicgende Ertrags: tufel II ift fiemad) ganz in ber Wasip, wie bie Ertragstafel I von mix fonitruicert worben, und will icf) nun ferourfeben, daj bic Epalte h

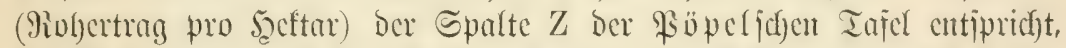

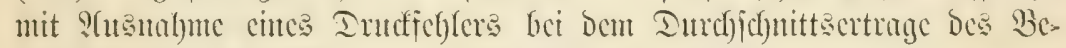
itandsalters 20 , weldyer 15,5 und nicht 16,5 beträgt.

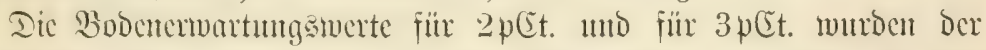

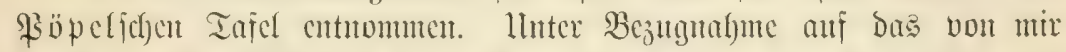
bet Ertragstafel I (Bcjagte halte id) weitere Erörterungen nidf)t fïr nütig.

\section{$\S 4$.}

\section{(Ertragetnid III nadj Edjwapwad).}

Dicje Tafel für dic Sitefent I. Bonitüt ijt Dem trefflichen $23 \mathrm{crfe}$ Des

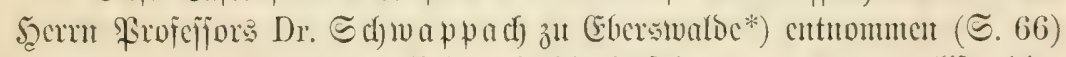

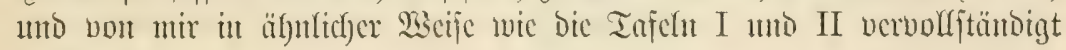
wordent.

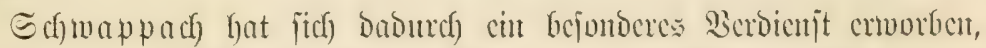

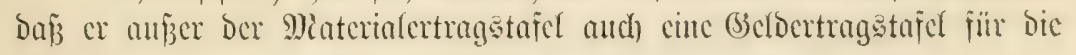

*) Wadgtum uno Ertrng normaler fiefernteftünte in ber norbbentiden Tiefelene. 


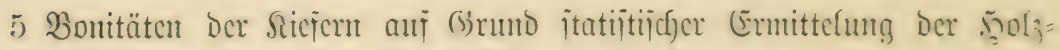
preije, Sultur= umo Berwaltumgsfoiten entworien hat.

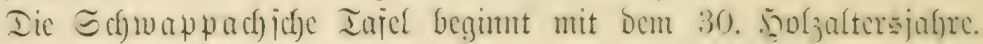

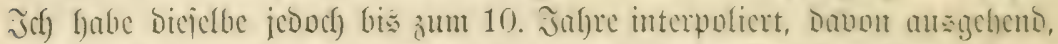

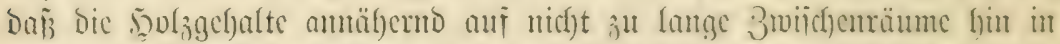

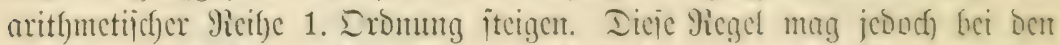

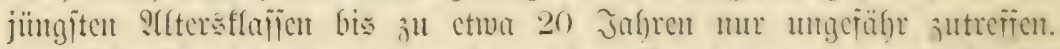

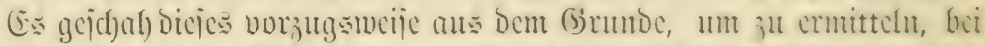

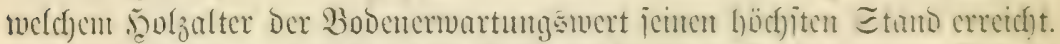

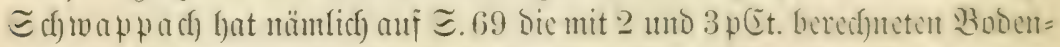

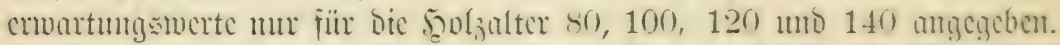

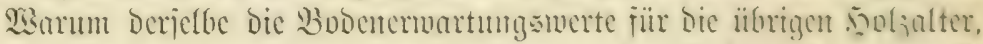

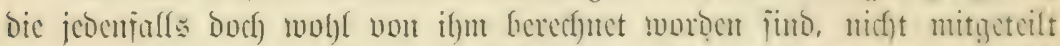

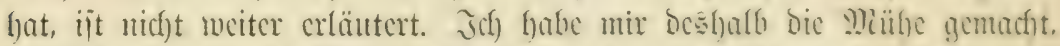

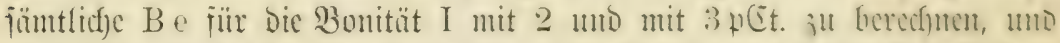

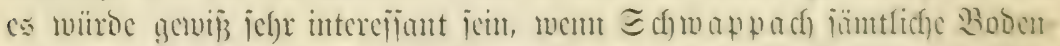

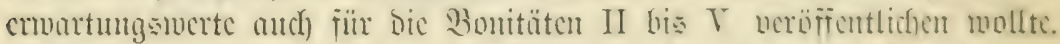

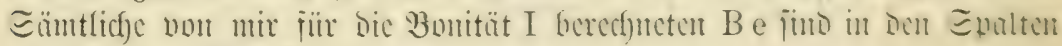
n uno o ber Tnbelle III cingetragen.

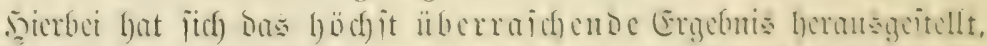

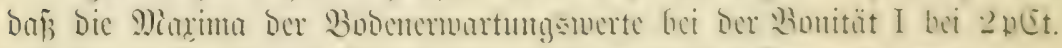

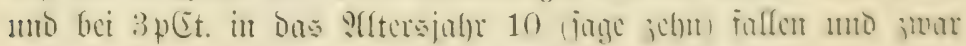

bci 2 pet. mit 2565 ell pro sigtar

" 3 " " 1598 " "

Iic un $\approx$ (f)

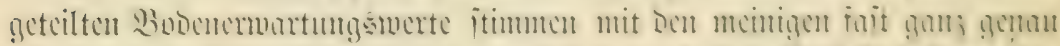

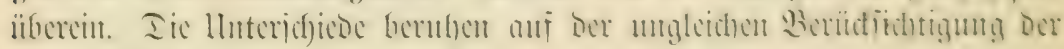
Dezimalitellen.

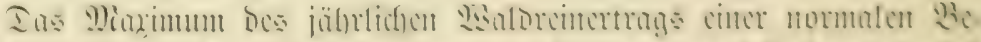

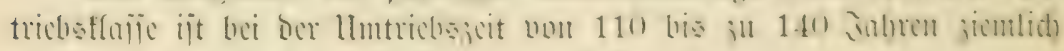

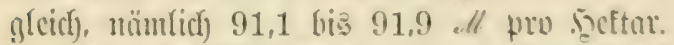

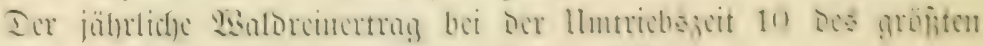

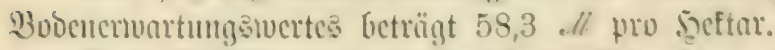

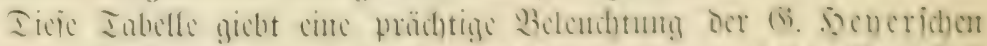

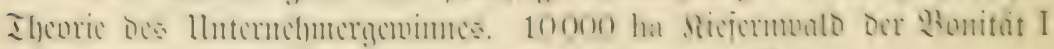

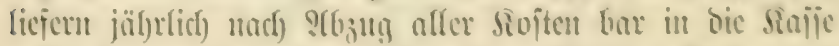

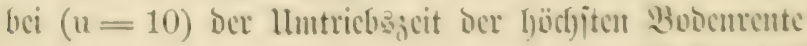

i) $8,3 \times 10000=583000$

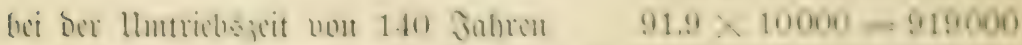
mithiut bei ledsterer mehr 336000 


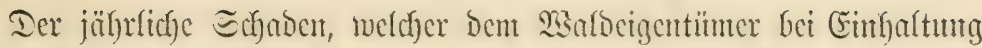

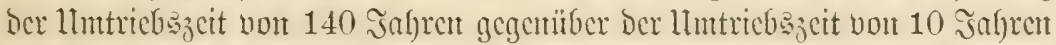

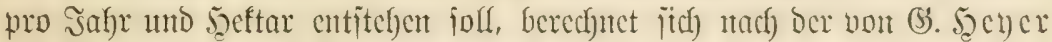

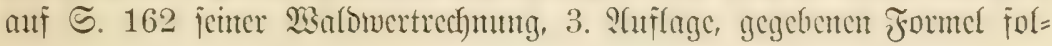
gentermâ̄en.

Diejelbe lautet:

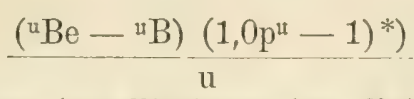

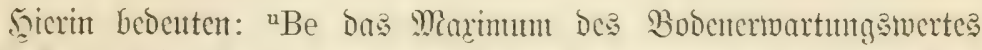
pro Sceftar bet ber llmtricbazcit u, "B Den Bobenwert ciner anderen llm= triebszeit it.

In umjerem Beippiele ijt mithin bei 3 pert.:

"Be bei ber Umtriebazcit $10=1598$ Al "B

$$
\begin{aligned}
& 140=197 " \\
& \text { "B } \mathrm{e}-{ }^{4} \mathrm{~B}=1401 \%
\end{aligned}
$$

Daher:

$$
1,03^{\text {u }}-1=1,03^{140}-1=61,69
$$

Daljer für 10000 ha $=6,173,406 \%$.

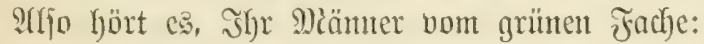

Went Shr 10000 ha Riefern Der I. Bontiä in Der Um=

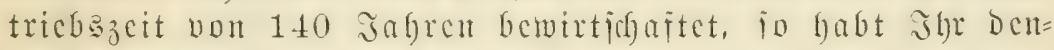

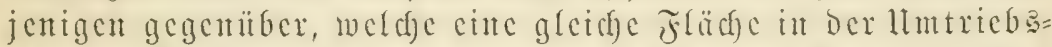
zeit der gröpten Bobentente von 10 Jahren bewirtidaften,

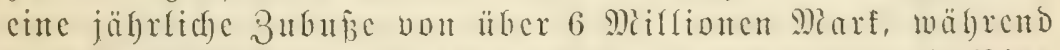
Dod) thatjüclich bic critereu am ङ von 336000 ell ben lebeteren gegenübcr bar in Siaje haben.

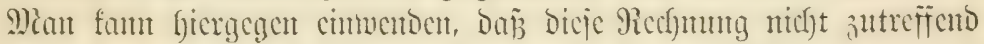

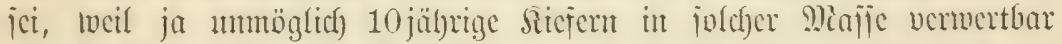

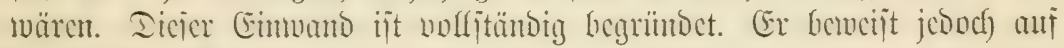
Das idflagendite:

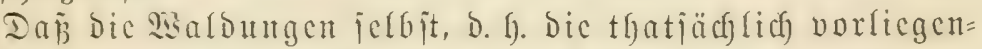

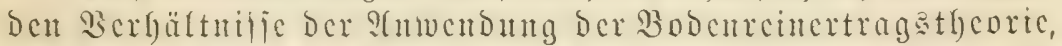

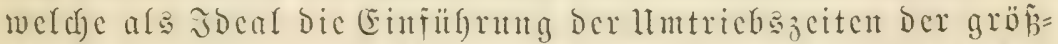
ten Bodenrenten aufitelt, cin gebieterifdes "rolt!" zutufen.

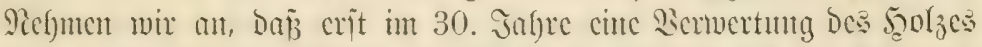

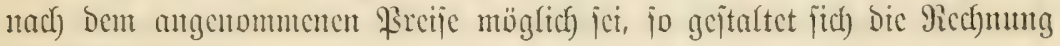
folgenbermá̧ent:

*) In bem februnryefte bes $B$ a uriden Eentralelattes you 1889, ๔. $8 \subseteq$, habe

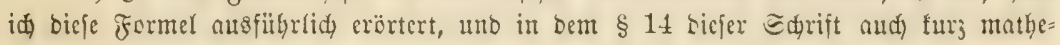
matifí) entwiféft. 


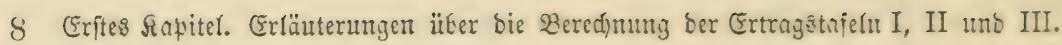

Sdjaben pro Seftar.

$$
\begin{aligned}
& \text { "Be im 30. Jahre bei } 3 \text { pČt. }=1263 \text { M } \\
& \text { uB im } 140 .
\end{aligned}
$$

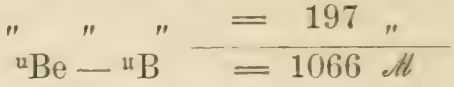

$$
\begin{aligned}
& \frac{1066 \times 61,69}{140}=\frac{65761,54}{140}=469,725 \% \text {. }
\end{aligned}
$$

Dafycr fïr 10000 ha

$$
10000 \times 469,725=4697250
$$

aljo über $4 \frac{1}{2}$ Millionen Mlarf.

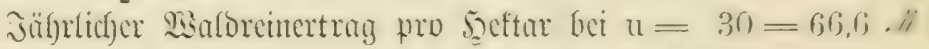

$$
\begin{aligned}
" \mathrm{u}=140 & =91,9 " \\
\text { Dififeren } & =25,3 \text { dl }
\end{aligned}
$$

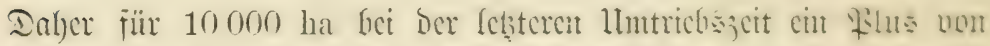

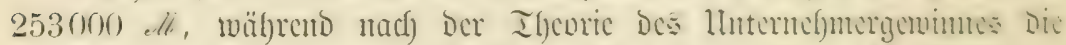

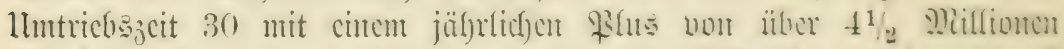

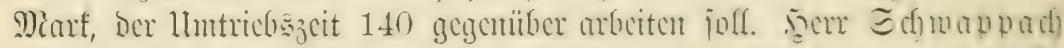

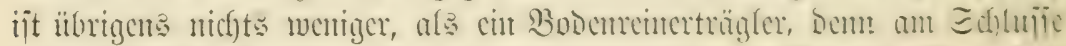

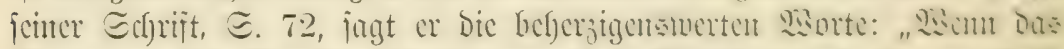

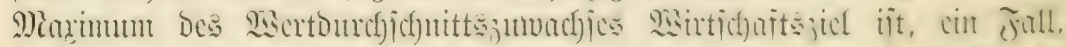

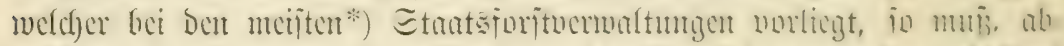

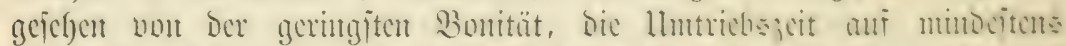

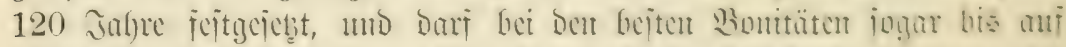

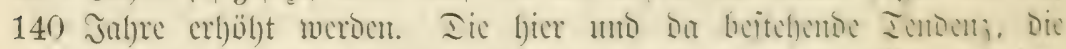

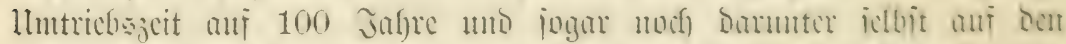
befferen Bonitüten forably

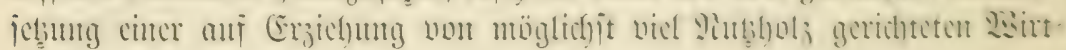

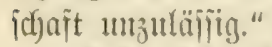

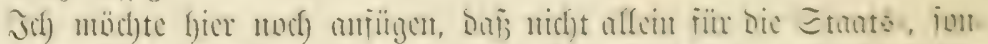

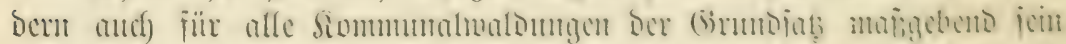

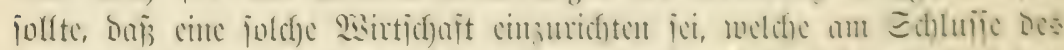

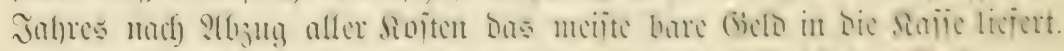

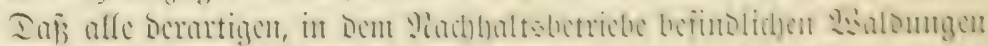

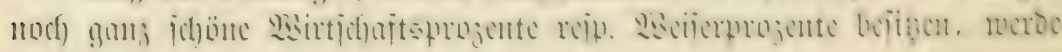
id) ipäter maffuncipen.

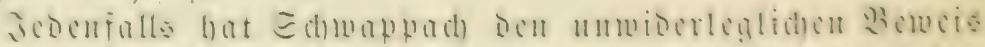

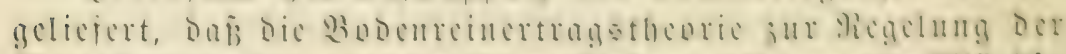

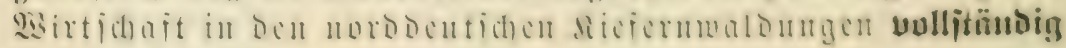

\section{murmat) bne ipt.}

*) 3d midute fagert: Eci allem. 
Ertragstafel I.

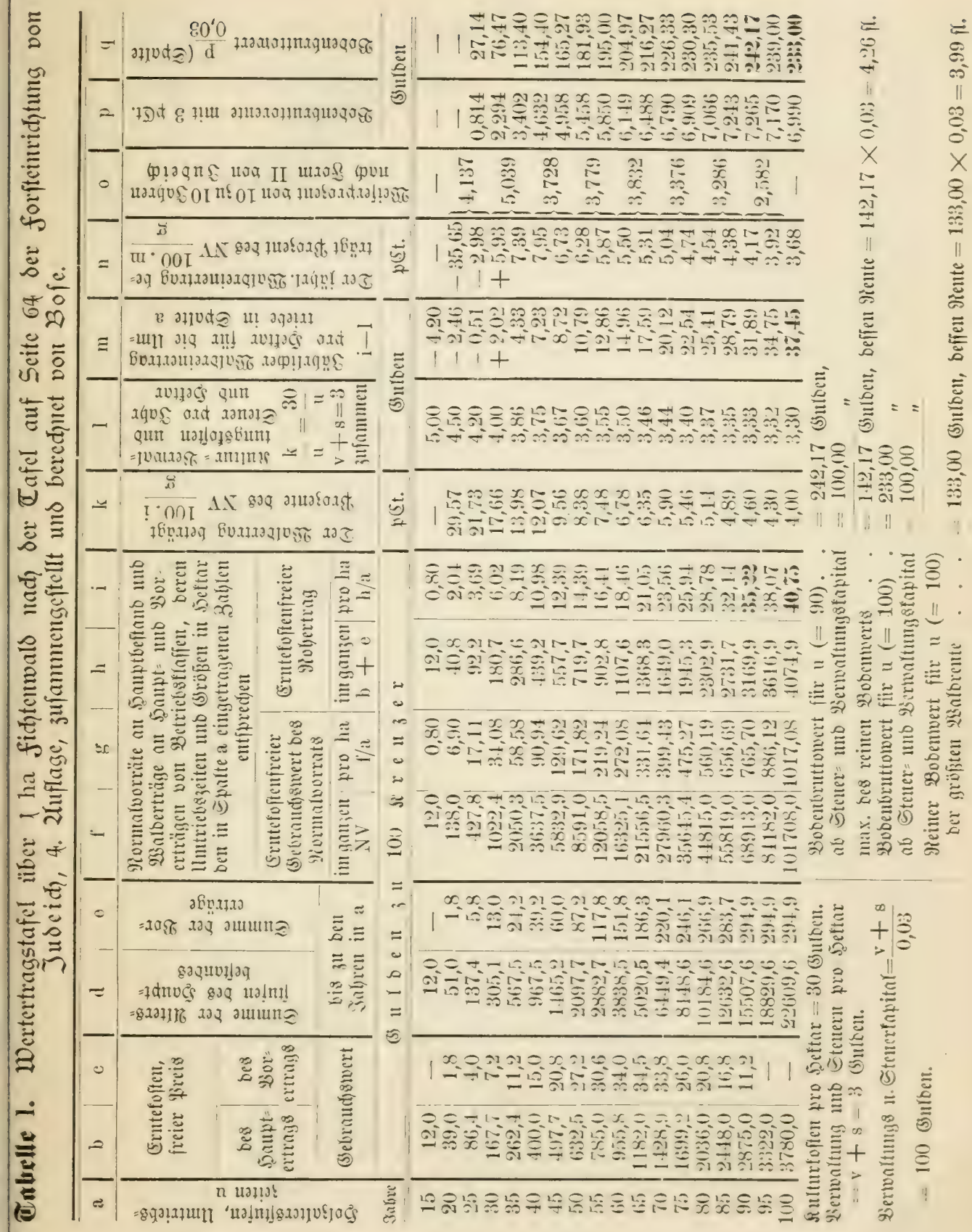




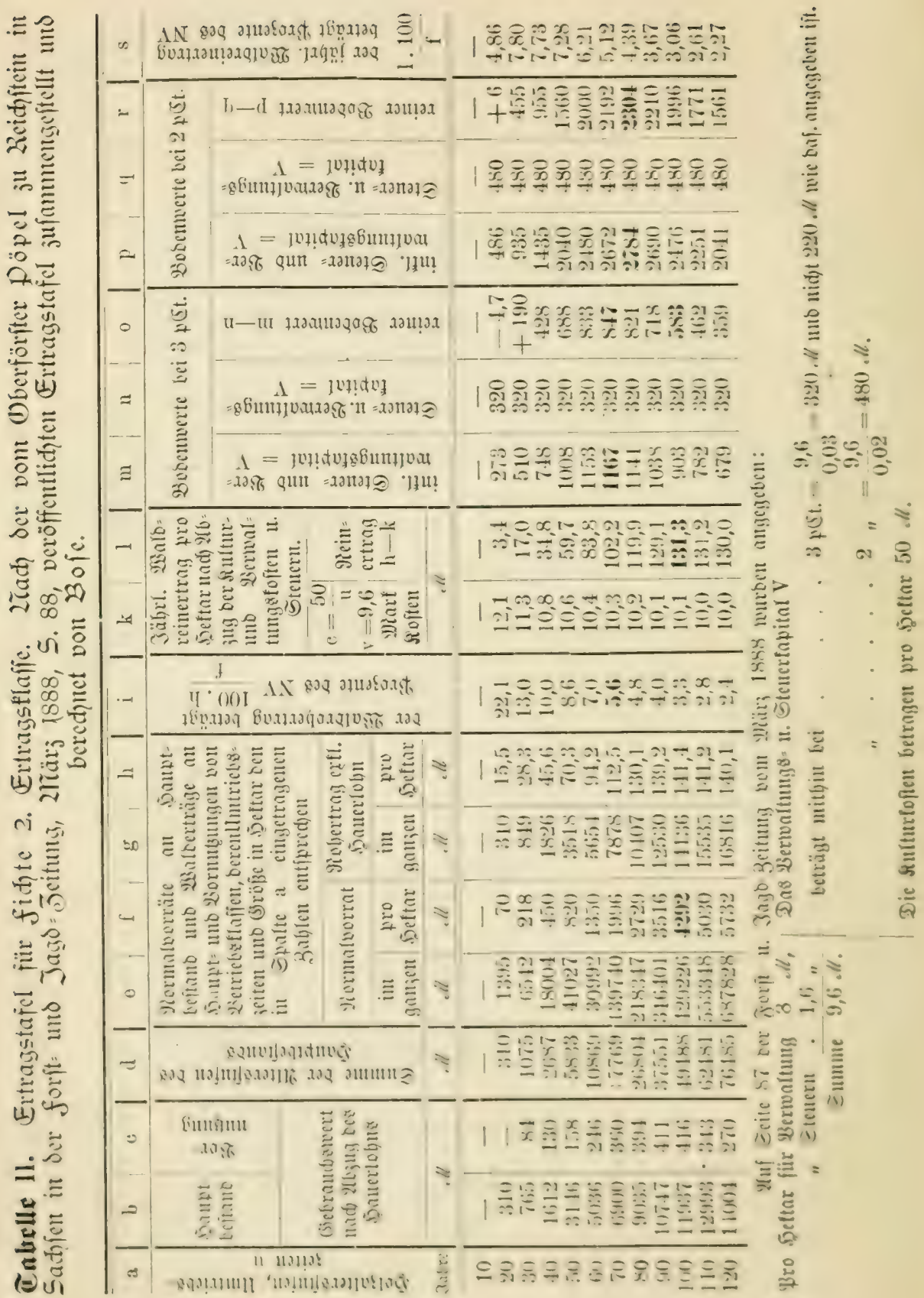




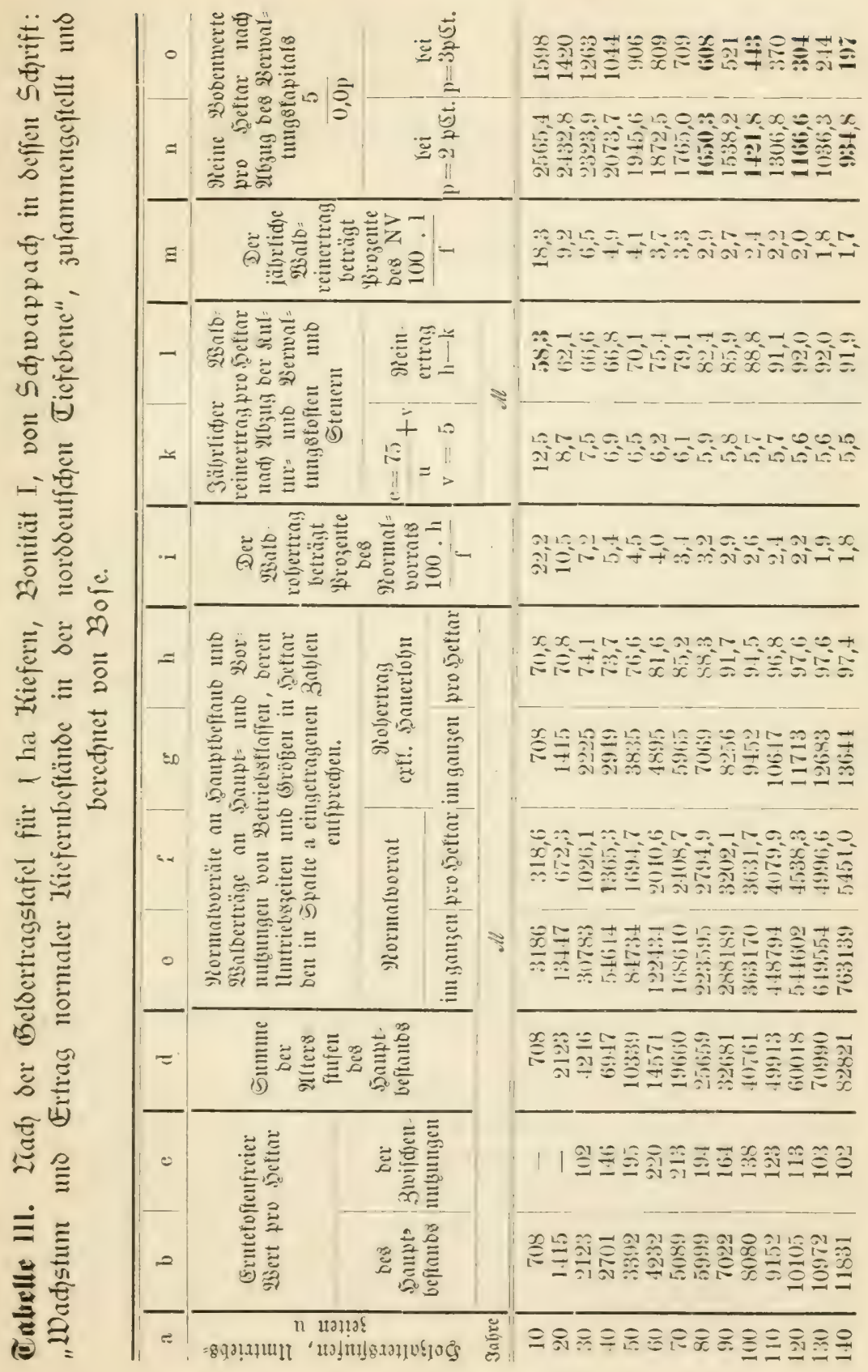




\section{כweites Kapitel. \\ Das forftlidje ascilerprozent.}

\section{A. BEgriff unû Einleifung.}

$\$ 5$.

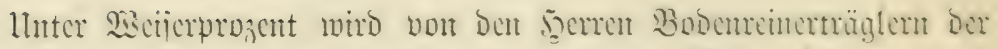

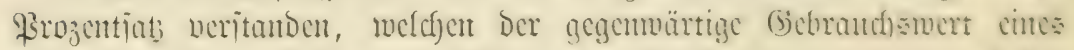

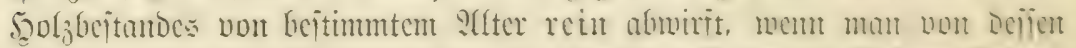

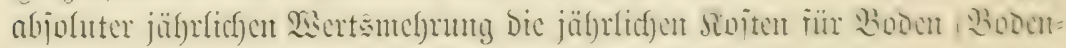
rente), Sierraltung unt Eteuern in S. (bzutg bringt.

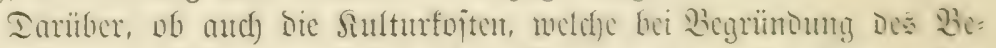

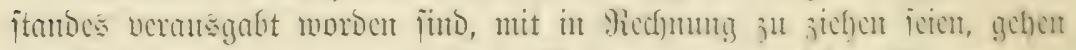

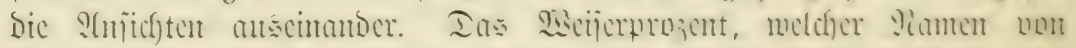

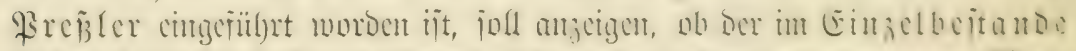

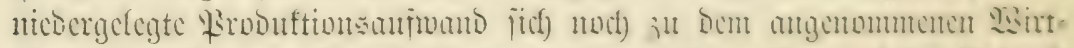

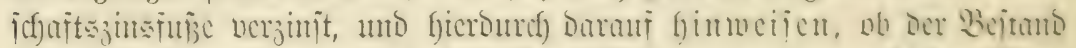

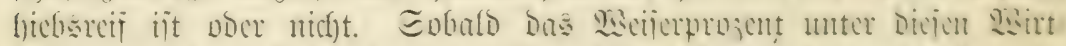

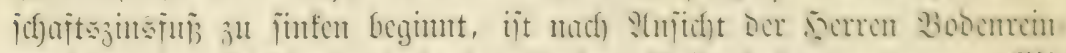

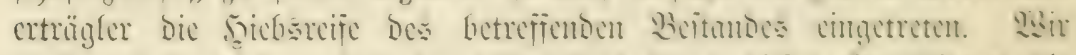

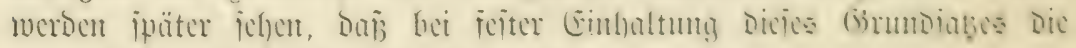

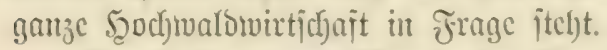

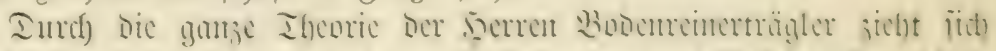

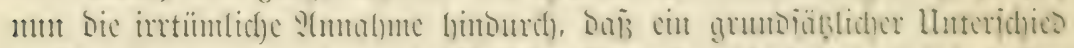
s)

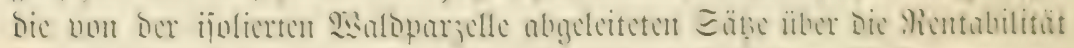

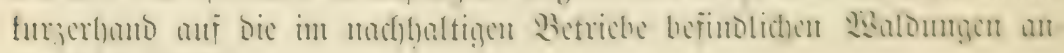
gewertioct iverbert.

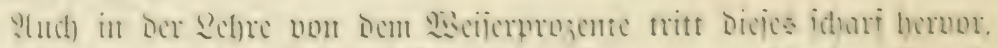

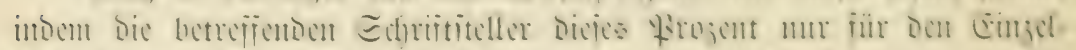

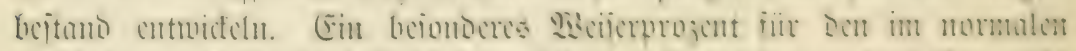

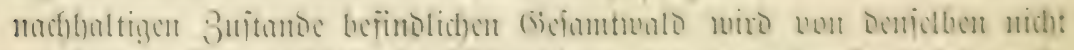

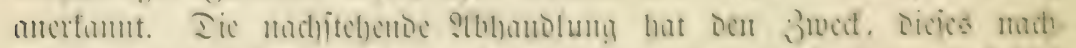

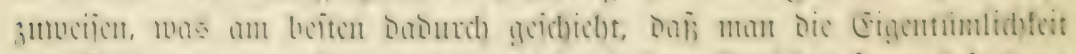

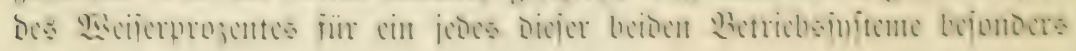
crörtert. 


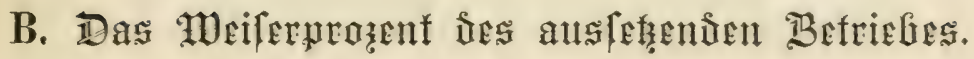

$\S 6$.

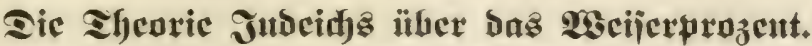

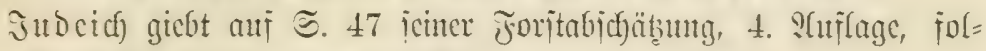

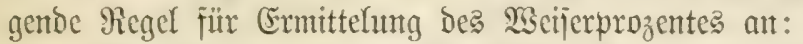

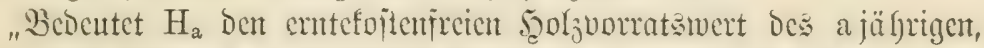

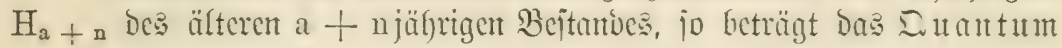

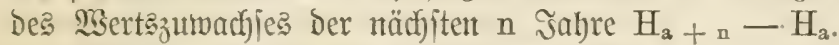

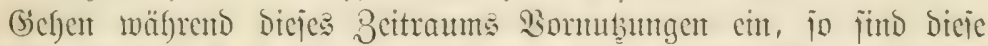

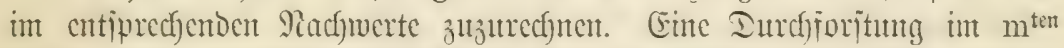

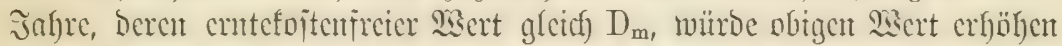
auf: $\left.\mathrm{H}_{\mathrm{a}}+\mathrm{n}+\mathrm{D}_{\mathrm{m}} \cdot 1,0 \mathrm{p}^{2}+\mathrm{n}-\mathrm{m} *\right)-\mathrm{H}_{\mathrm{a}}$ twent ber antgenommenc $\mathfrak{s i r t}=$

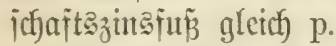

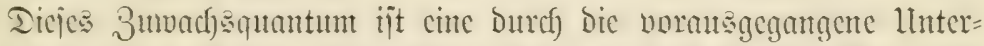

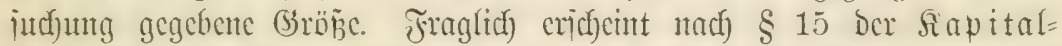

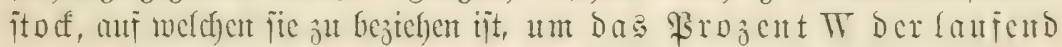
jägrliçen Berzinjung zu finden.

Besectefnet man bie Eumme ber im vorigen Paragraph entrutefeltent brei

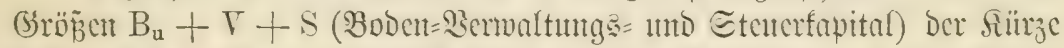
halber mit $g$, jo repräjentiert biejes $g$ cinen Siapitatwert, Dejien Zimjen

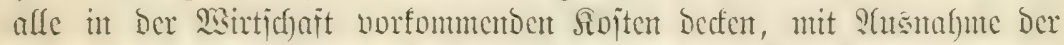

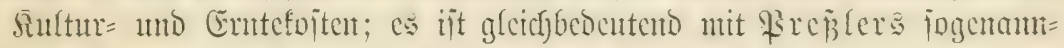
tem Bobenfrutto=Sapitale. Eicics g mun unter allen llmjtänden ben cinen Teil bee fraglichen Siapitalitocfes bildoen."

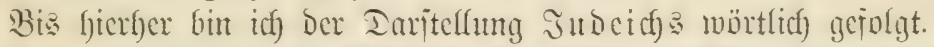

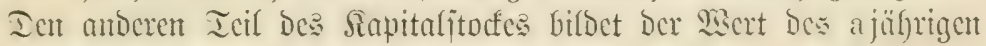

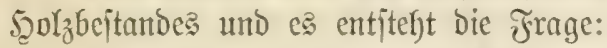

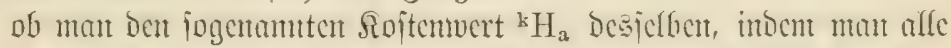

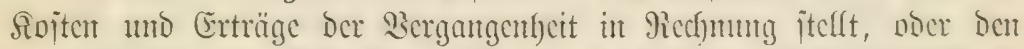

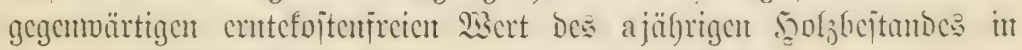
Die Formel einzufüffren lyabe.

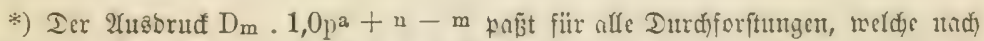

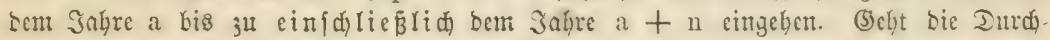
forftung in bem Sabre $(a+n)$ ein, fo wirb $m=a+n$, unb ber $23 e r t$ ber Durd $=$ forfung in bem $a+n^{\text {ten }}$ Sabre iff bann $\left.=D_{a}+n \cdot 1,0 p^{(a}+n\right)-(a+n)=$ $\mathrm{D}_{\mathrm{a}}+\mathrm{n} \cdot 1,0 \mathrm{p}^{0}=\mathrm{D}_{\mathrm{a}}+\mathrm{n}$.

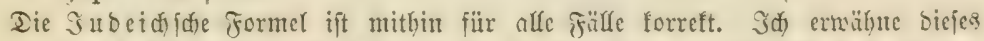

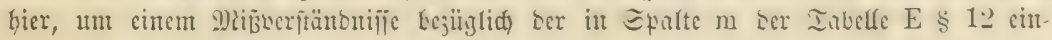
getragenen Formel borjubetgent.

Boje. 


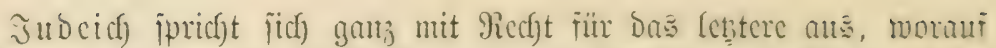

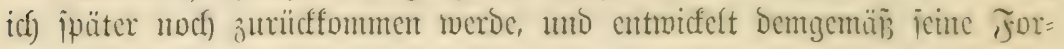
mel II auf S. 49.

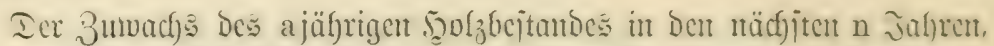

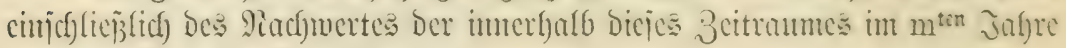
crjolgten Durchjurjtumg beträgt mitbiu:

$$
\mathrm{H}_{\mathrm{a}+\mathrm{n}}+\mathrm{D}_{\mathrm{m}} \cdot 1,0 \mathrm{p}^{\mathrm{a}}+\mathrm{n}-\mathrm{m}-\mathrm{H}_{\mathrm{a}} \text {. }
$$

Iicjem Zumadjic jteft cin Siapitalitod gegenilfor vou $\mathrm{B}_{\mathrm{u}}=$ reiner

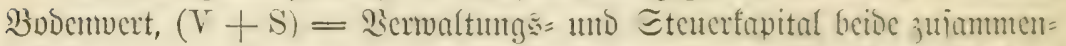

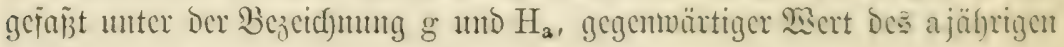
50 zabejtande?.

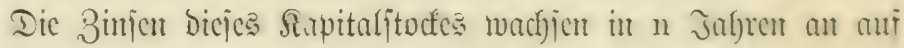

$$
\left(\mathrm{H}_{\mathrm{a}}+\mathrm{g}\right)\left(1,0 \mathrm{w}^{\mathrm{n}}-1\right) \text {, }
$$

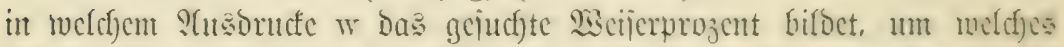

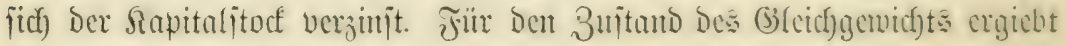
fich) Gierans bic Fonmel:

$$
\mathrm{H}_{\mathrm{a}+\mathrm{n}}+\mathrm{D}_{\mathrm{m}} \cdot 1,0 \mathrm{p}^{\mathrm{a}}+\mathrm{n}-\mathrm{m}-\mathrm{H}_{\mathrm{a}}=\left(\mathrm{H}_{\mathrm{a}}+\mathrm{g}\right)\left(1,0 \mathrm{w}^{\mathrm{n}}-1\right)
$$

Ђeicralts

$$
\begin{gathered}
1,0 w^{\mathrm{n}}-1=\frac{\mathrm{H}_{\mathrm{a}}+\mathrm{n}+\mathrm{D}_{\mathrm{m}} \cdot 1,0 \mathrm{p}^{\mathrm{a}}+\mathrm{n}-\mathrm{m}-\mathrm{H}_{\mathrm{a}}}{\mathrm{H}_{\mathrm{a}}+\mathrm{g}} \\
1,0 \mathrm{w}^{\mathrm{n}}=\frac{\mathrm{H}_{\mathrm{a}+\mathrm{n}}+\mathrm{D}_{\mathrm{m}} \cdot 1,0 \mathrm{p}^{2}+\mathrm{n}-\mathrm{m}-\mathrm{H}_{2}}{\mathrm{H}_{\mathrm{a}}+\mathrm{g}}+1 \\
1,0 \mathrm{w}^{\mathrm{n}}=\frac{\mathrm{H}_{\mathrm{a}+\mathrm{n}}+\mathrm{D}_{\mathrm{m}} \cdot 1,0 \mathrm{p}^{\mathrm{a}}+\mathrm{n}-\mathrm{m}+\mathrm{g}}{\mathrm{H}_{\mathrm{a}}+\mathrm{g}}
\end{gathered}
$$

Form. II, W $=100\left[\frac{\mathrm{n}\left(\mathrm{H}_{3}+\mathrm{n}+\mathrm{D}_{\mathrm{m}} \cdot 1,\left(1 \mathrm{I}^{\mathrm{a}}+\mathrm{u}-\mathrm{m}+\mathrm{c}\right.\right.}{\mathrm{H}_{3}+\mathrm{m}}-1\right]$

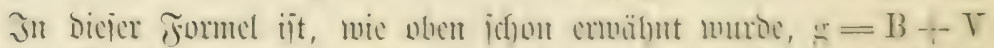

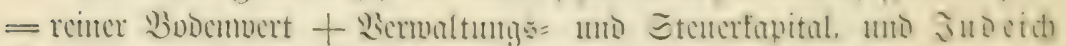

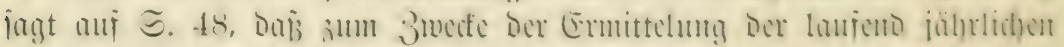

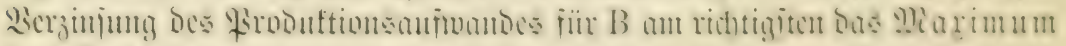
des Bodencruartugalucrtes $=B_{u}$ cingefübrt ucroc.

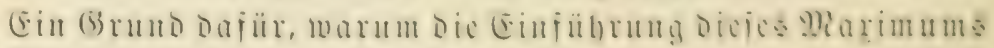
Das Sichtigfte fei, wiro nidft angegefon.

$$
\text { (Sicke bic Inbelle A mij ङ. 15.) }
$$

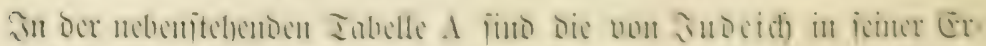

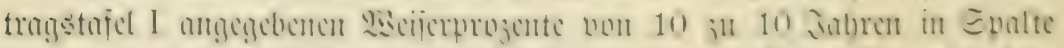

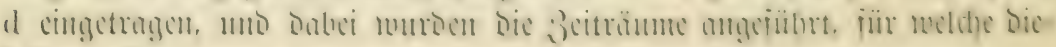
jelloen gelten. 
B. In\& Meijerfrojent bes auฐjekenben Betriebes. $\$ 6$.

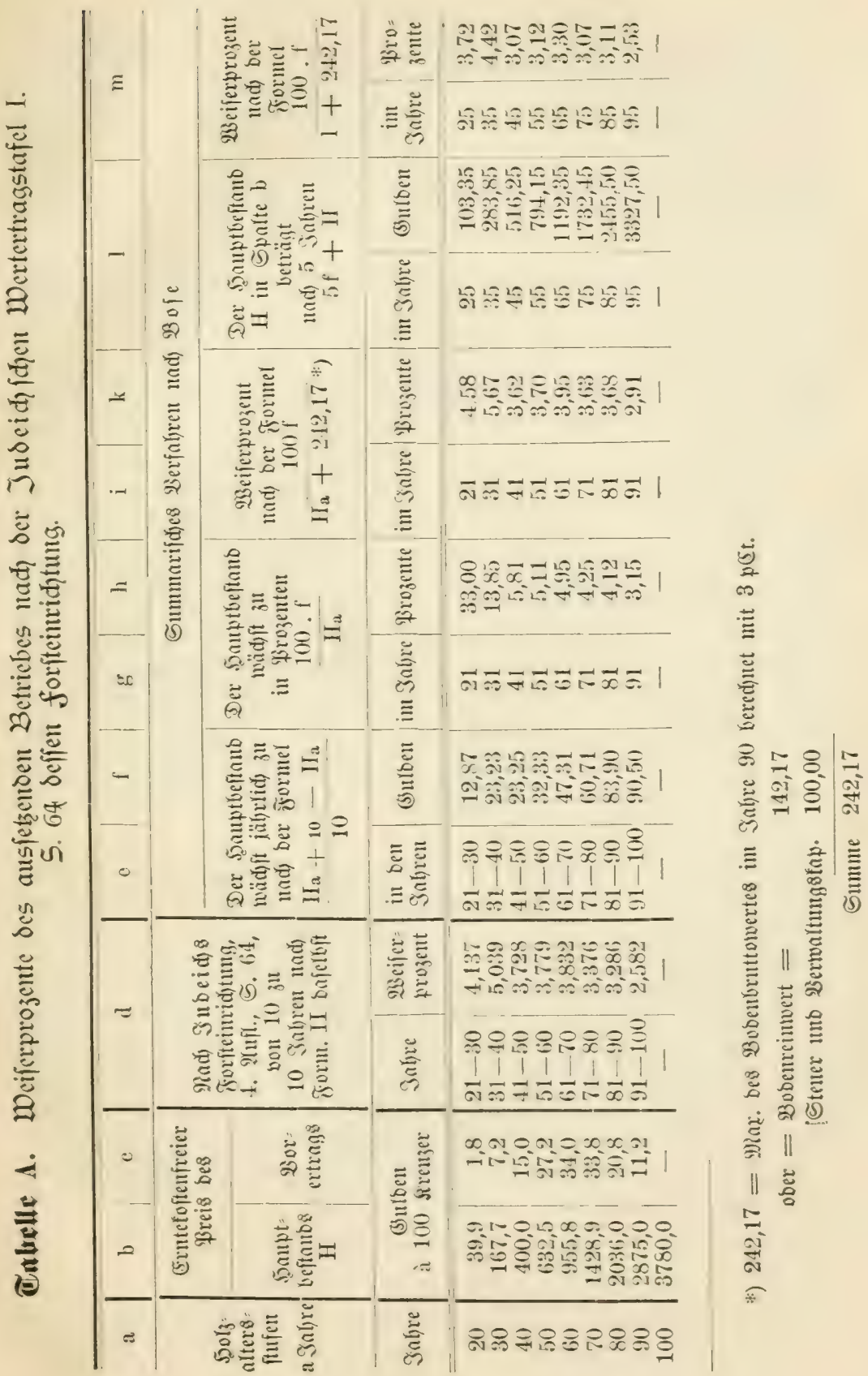




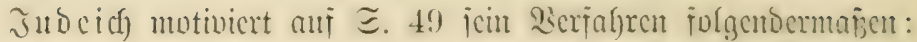

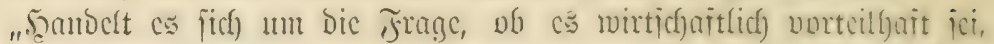

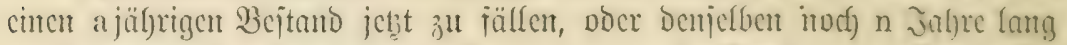

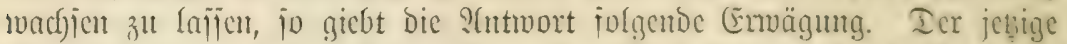

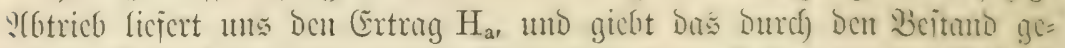

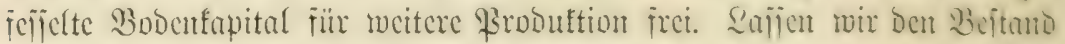
nod) n Jabre ftchen, jo licjert or ben Ertrag $\mathrm{H}_{\mathrm{a}}+\mathrm{n}$, Gcjichungsuciic $\mathrm{H}_{\mathrm{a}}+\mathrm{n}+\mathrm{D}_{\mathrm{m}} \cdot 1,0 \mathrm{p}^{\mathrm{a}}+\mathrm{n}-\mathrm{m}$.

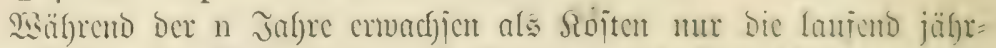

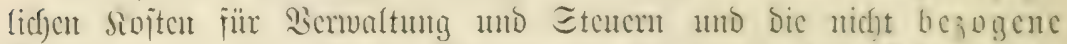

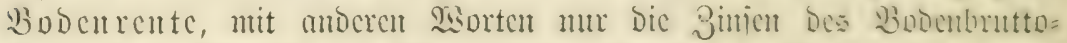

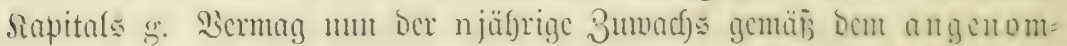

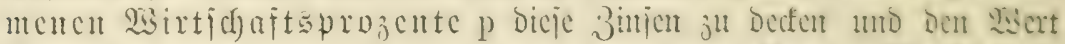

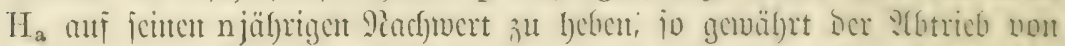

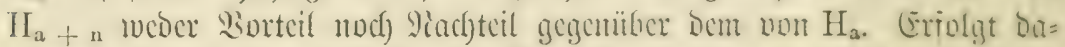

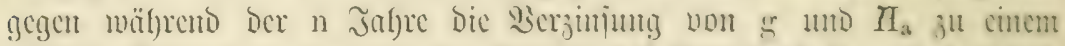

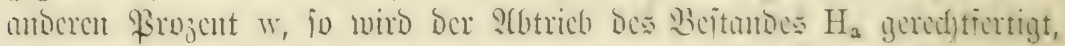

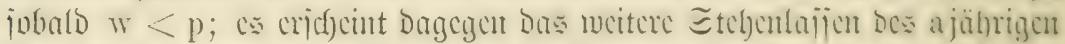

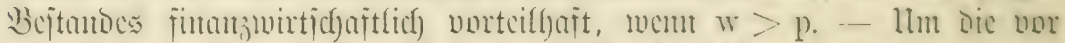

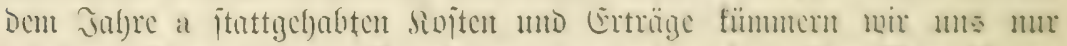

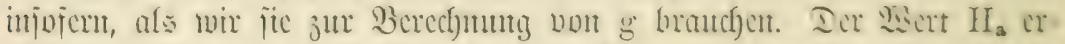

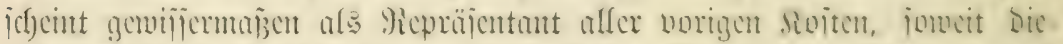

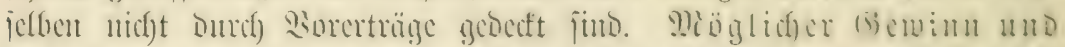

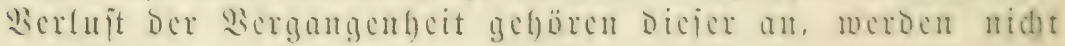
auf die $3 u t u f t$ übertragen.

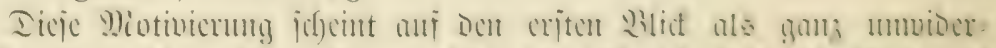

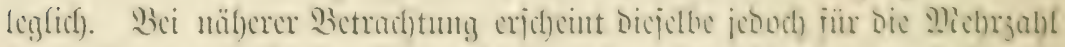

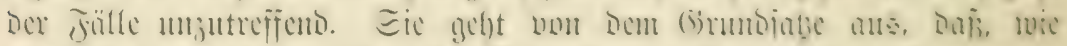

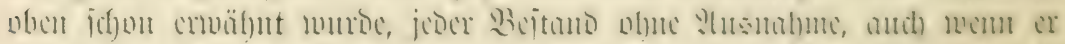

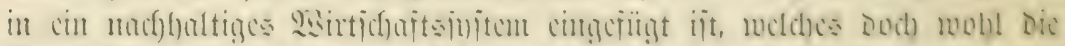

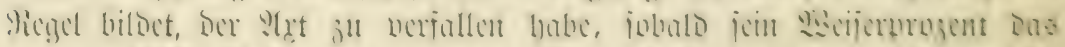

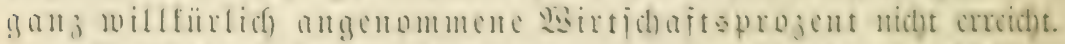

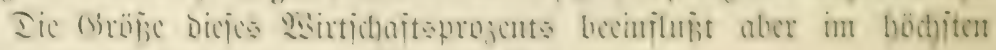

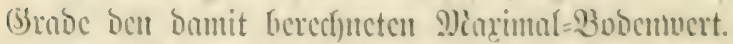

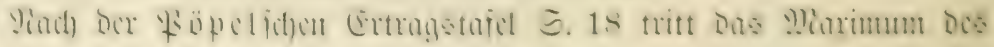
Woocuforittonertes cin:

bei 3 pert. in 70. Sulfe mit 1167 .ll

" 2. " 80. " " 2784

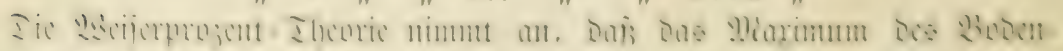

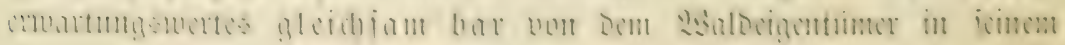




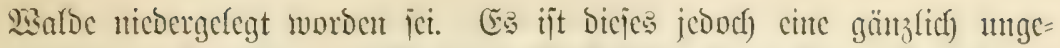

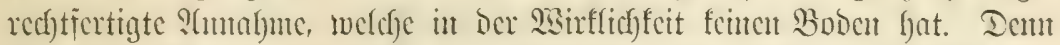

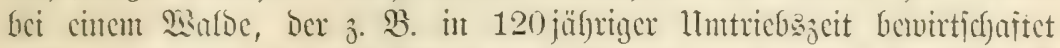

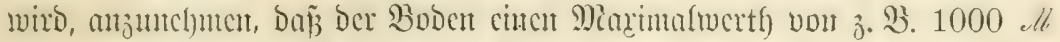

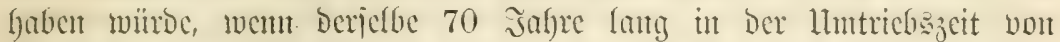

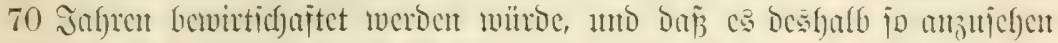

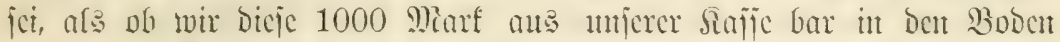

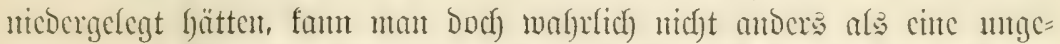

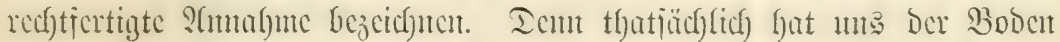

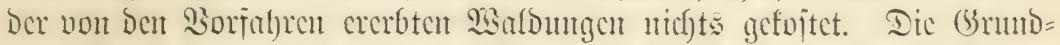

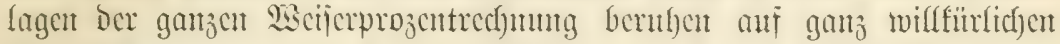

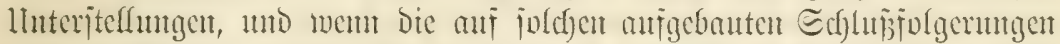

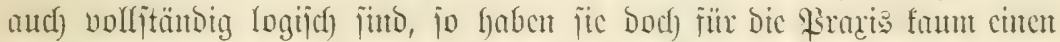
2.

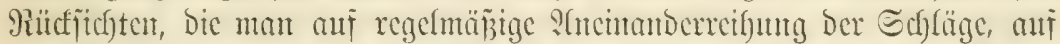

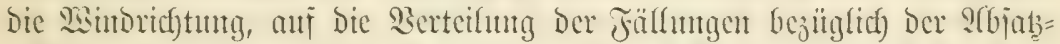
gebicte x. ju nel)men lat, viel widftiger als bas 2 seciferprozent finto. Iieje

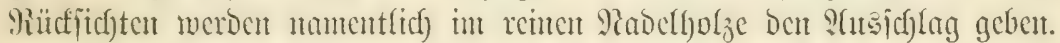

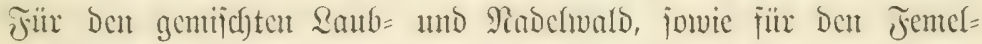

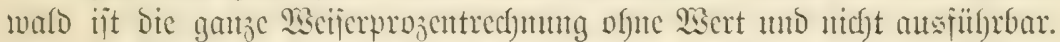

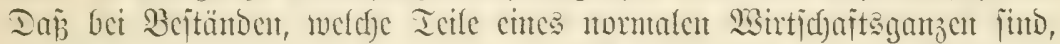

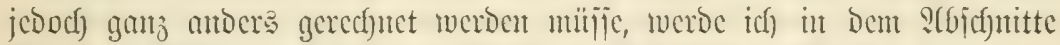
Ïber Das 2 scijerprozent bes nadylyaltigen Betricbes nadjweijen.

$\S 7$.

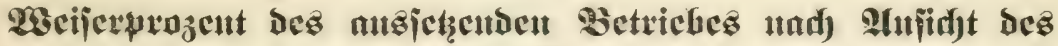
Berijnipers.

Ias von mix in ber mitgeteiften Iabeffe A angegebene fummarijde

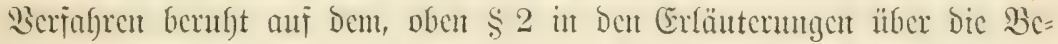

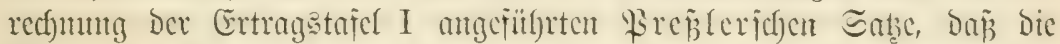

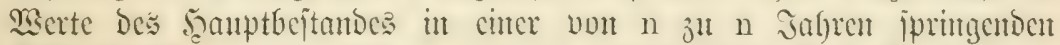

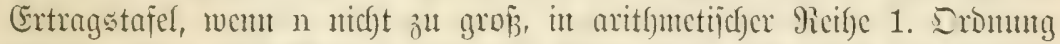

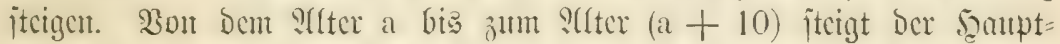

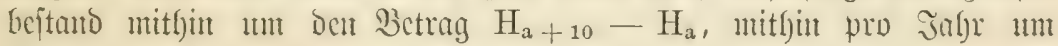

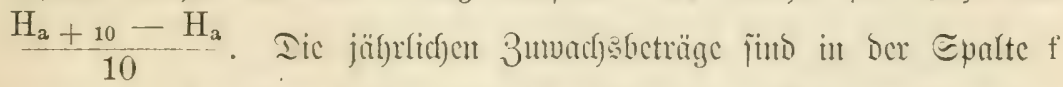
angegebon. Das 3uwad)

$$
\frac{\left(\frac{\mathrm{H}_{\mathrm{a}+10}-\mathrm{H}_{\mathrm{a}}}{10}\right) 100}{\mathrm{H}_{\mathrm{a}}}=\left(\frac{\mathrm{H}_{\mathrm{a}}+10-\mathrm{H}_{\mathrm{a}}}{\mathrm{H}_{\mathrm{a}}}\right) 10
$$




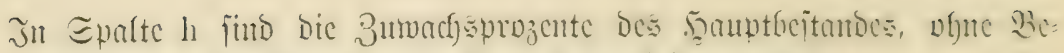

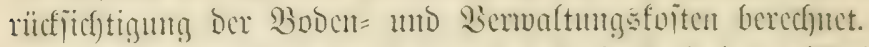

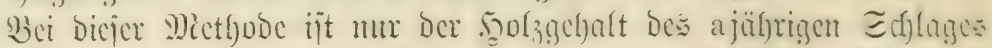

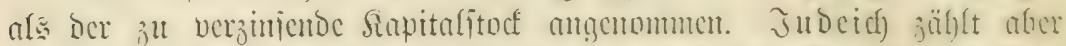

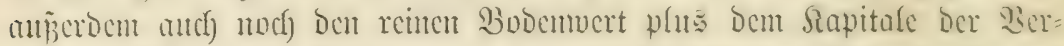

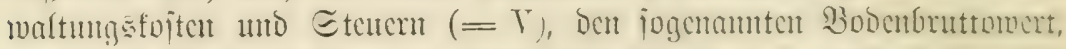

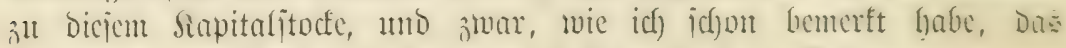
Marimum von Be, im vorlicgenda falle $=242,17$ (binlout.

Die von mir gegebene Formel wïroe ljiernad) lauten:

$$
W=\frac{\left(H_{2}+10-H_{a}\right) 10}{H_{a}+B e+V}
$$

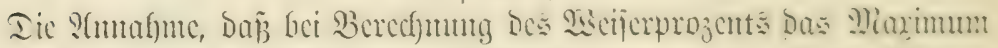

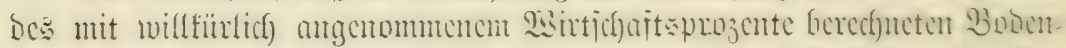

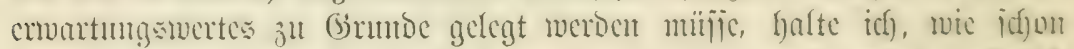

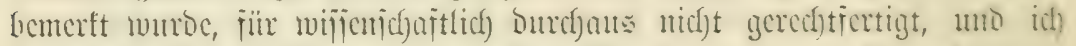
l)abe bestgalb bas mBe mu ans bem Grumbe it meine formel cinge

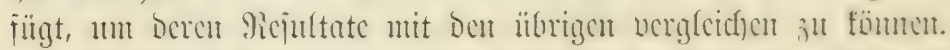

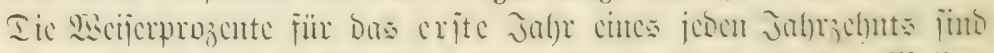

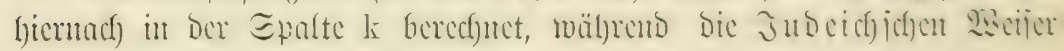
prozente in Epalte d im Durdfjeltitte für 10 Jabre geltent.

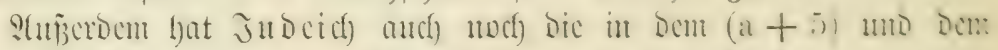

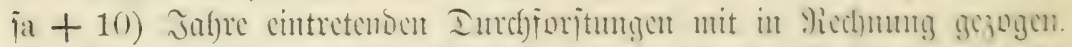

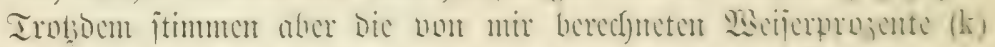

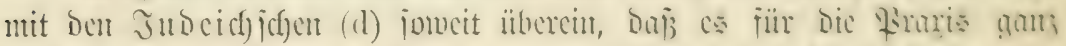

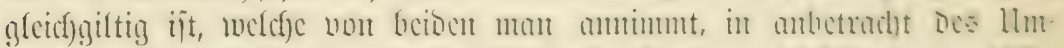

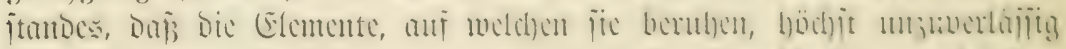

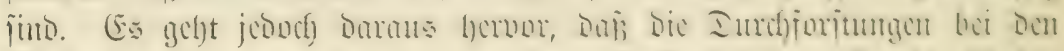

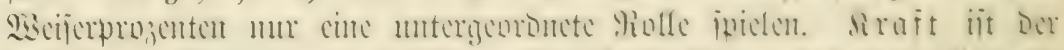

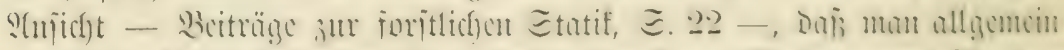

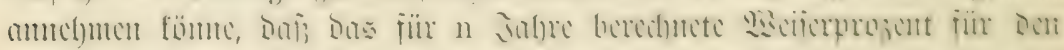

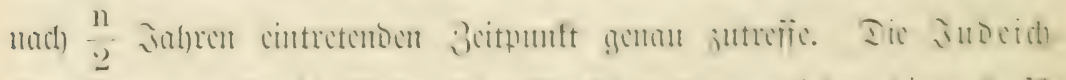

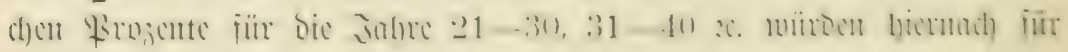
Dic Inl)re 25,35 x. gुclten.

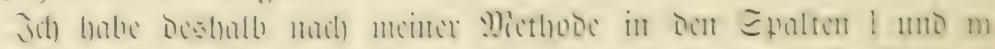

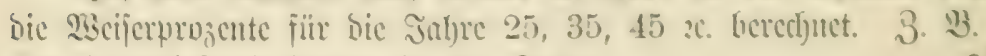
Der 5authefitumb betrïgt im 60. Jalure 955,80

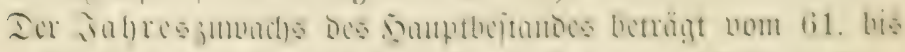

70. Jafure 47,31. Daffer fitr 5 Jaf)re $=47,31 \times 5=236,55$

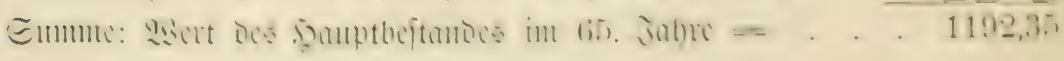




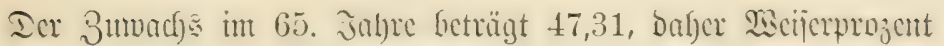

$$
\frac{100 \times 47,31}{1192,35+242,14}=3,30
$$

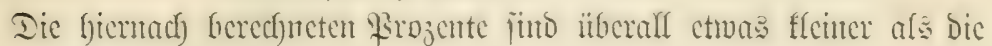

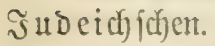

$$
\$ 8 \text {. }
$$

\section{Beijerprozente bes atsietsenton Betricbes ber Pöpclidjent Ertragstajel (II) แad ber vben crwäljuten Ittocidjodjen Formel II Geredjut.}

(Siebe bie Tabelle B auj ऽ. 20.)

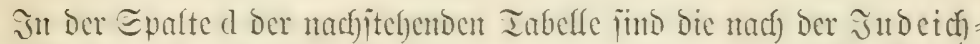

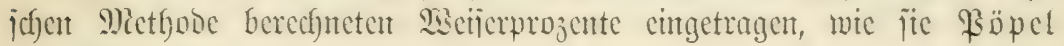
angegeben hat.

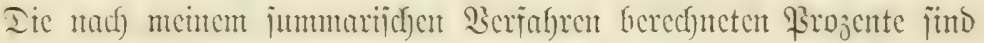
in ben Epalten e biz $\mathrm{k}$ berectinet uno eingetragen.

Iic in Epalte k, unter 3uzichung bes gröiten mit 3 pCEt. bc=

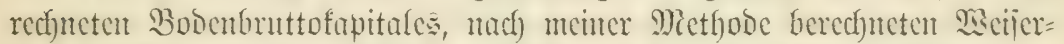

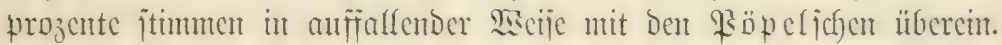

In Epalte 1 f̈mD Dic nad) meiner Metfode crmittelten Mecijer= projente unter ? bruttowertes cingetragen. Iicielfen weidfen vom 70 . Jafyre an mu

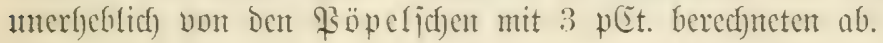

$\S 9$.

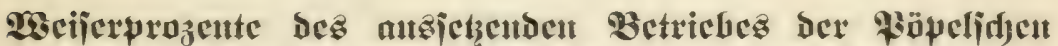

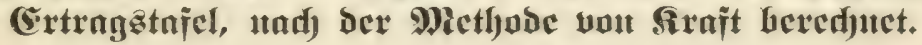

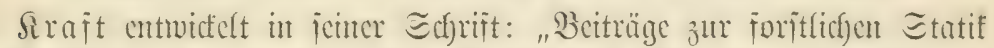

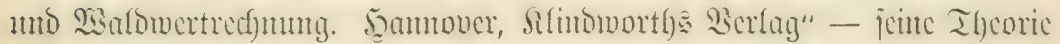

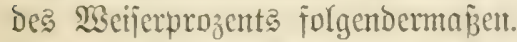

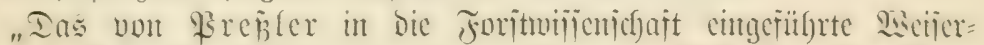

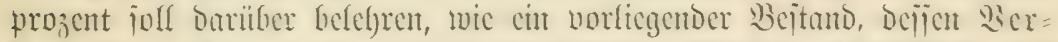

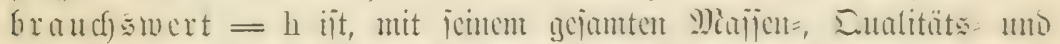

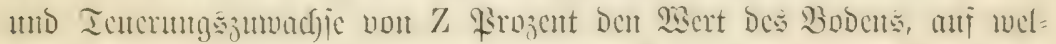

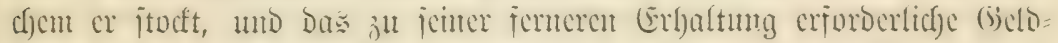

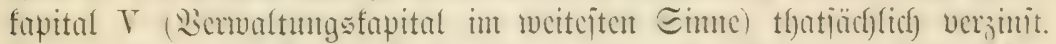

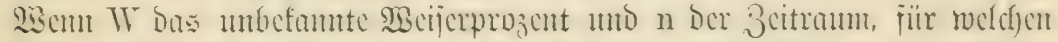

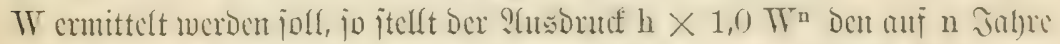
prolougierten Rettowert ber gejamten Reijtuntig bar. Iicjer Vectomert

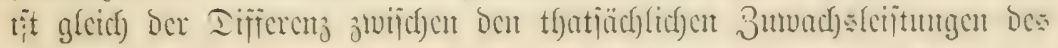




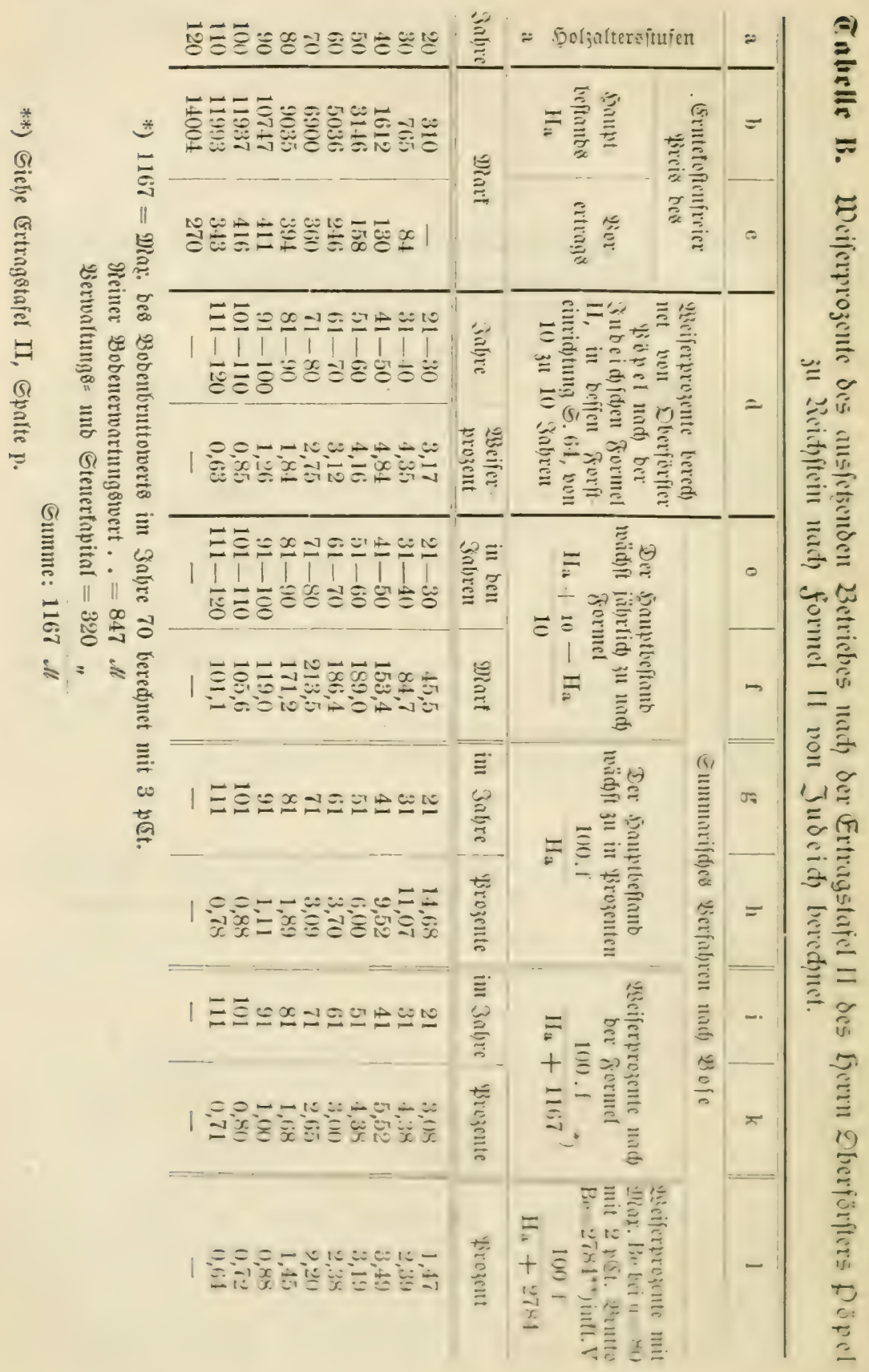


Bejtandes für $\mathrm{n}$ Jafure $=\mathrm{h} \times 1,0 \mathrm{Z}^{\mathrm{n}}$ แnd Den $\mathrm{n}$ jäfrigen Simjen Des

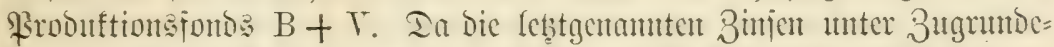

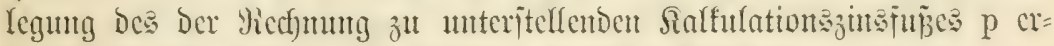
mittelt werben milfien, fo befommt man bie (Sfleicfung

woraus folgt

$$
\left.\mathrm{h} \times 1,0 \mathrm{~W}^{\mathrm{n}}=\mathrm{h} \times 1,0 \mathrm{Z}^{\mathrm{n}}-(\mathrm{B}+\mathrm{V})\left(1,0 \mathrm{p}^{\mathrm{n}}-1\right){ }^{*}\right)
$$

$$
\text { 1. } 1,0 \mathrm{~W}^{\mathrm{n}}=1,0 \mathrm{Z}^{\mathrm{n}}-\left(\frac{\mathrm{B}+\mathrm{V}}{\mathrm{h}}\right)\left(1,0 \mathrm{p}^{\mathrm{n}}-1\right) \text {. }
$$

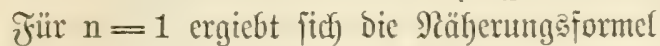

$$
\text { 2. } \mathrm{W}=\mathrm{Z}-\left(\frac{\mathrm{B}+\mathrm{V}}{\mathrm{h}}\right) \mathrm{p} . "
$$

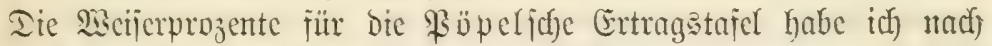

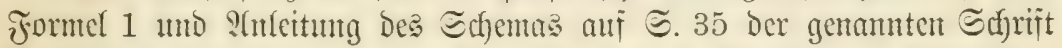
Srafts in ber beifolgenden Iabelle C in ben Spalten a-i berechnet.

(Siche Die Tabelle C alif 5.22. )

3ur Berecfuntg von $\mathrm{Z}$ und $\mathrm{W}$ aus $1,0 \mathrm{Z}^{\mathrm{n}}$ แnd $1,0 \mathrm{~W}^{\mathrm{n}}$ murbe bic

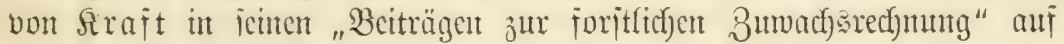
๔. 143 mitgeteilte Iafel bemubzt.

Die bctreffenden W fut in Epalte i cingetragen. Diejelben geltent fïr $\mathrm{n}=10$. Ias jum Beippiel bei bem ?llter 50 cingetragente $\pi$ gilt für Die 3eit von 51-60 ฐafren.

Da die Formeln von (3). Dener und Pref́ler, jorvie das vou mir

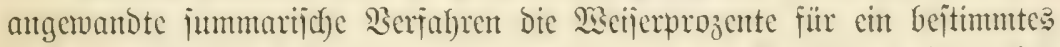
Jab) angeben, jo babe id) bicjelben audf) nad) ber Sraftichen formel 2 bereclynet.

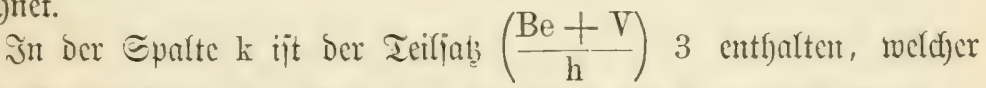
Durch) Diultiplifation ber Beträge in Epalte f mit 3 crmittelt murbe.

Tie in Epalte e cingetragenen 3uradisprozente Z vermindert $1 \mathrm{~m}$ Die Beträge in Epalte $\mathrm{k}$ crgeben bic Becijerprojente nad) Formel 2 in Der Epalte 1. Ticjelfen jtimmen vom 50. Jahje an nahezł mit ben nad) Formel 1 berechneten überein.

\section{$\S 10$.}

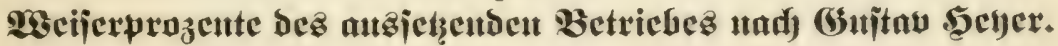

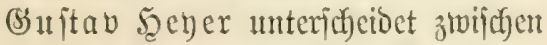

a) Der laujend jälbrliç)en und

b) Der Durchjictnittlich jüfrlicten

Berzinung des ßroduttionsfond:

*) $(B+V)$ madfjen mit Sinfeszinfer in n $\Im a b r e n$ an nuf $(B+V) 1,0 p^{n}$, vringt

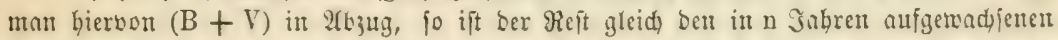
Bimien $=3$. Daler $3=(B+V)\left(1,0 p^{n}-(B+V)=(B+V)\left(1,0 y^{n}-1\right)\right.$. Bo 


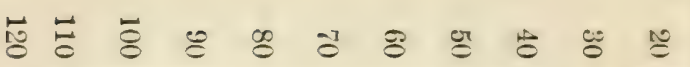

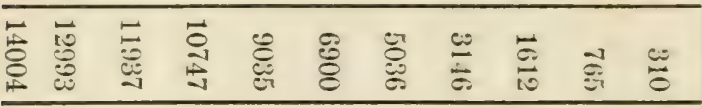

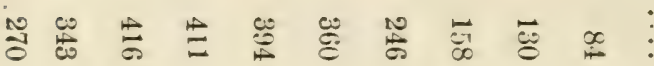

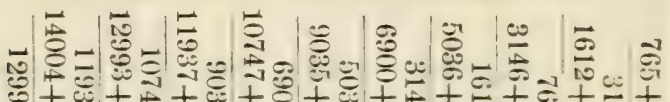

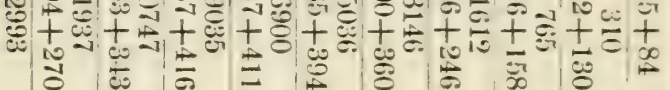

तै

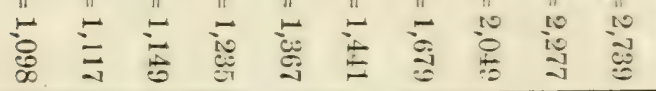

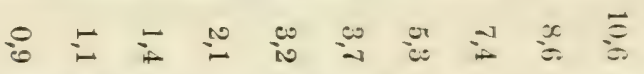

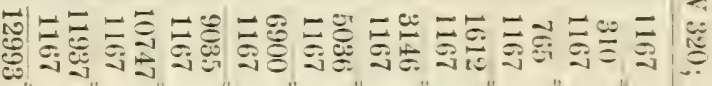
b. S 3umadare

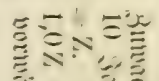

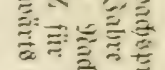

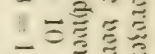
o以

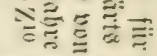
jent fiir 113abre = vormärts $Z$

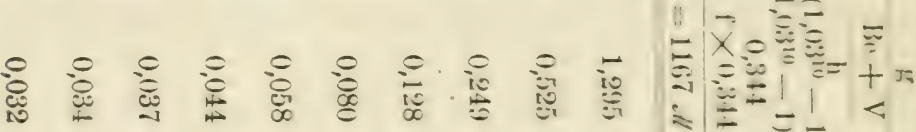

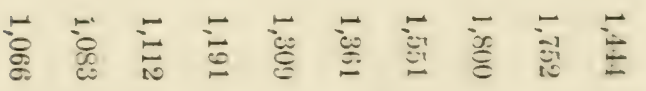

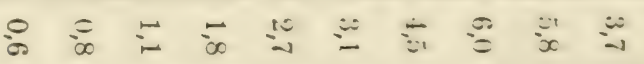

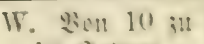

$\stackrel{\circ}{=}$

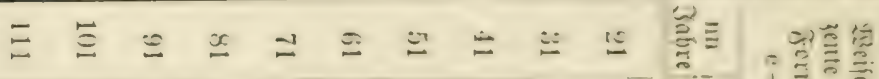

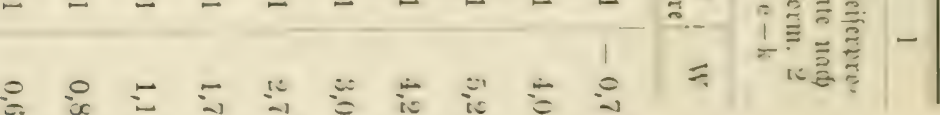




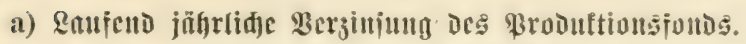

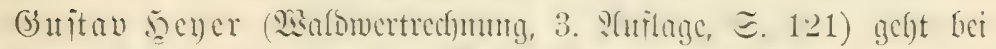

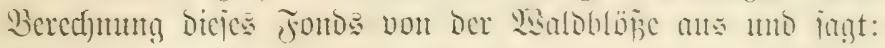

ber im Salye 0 vorbandene \$robuftionsionds bciteft aus

1. B, Den Bobenfapital,

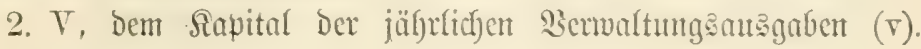
Daber $\mathrm{V}=\frac{\mathrm{r}}{0,0 \mathrm{p}}$,

3. Den bet Begrïnoung bes Bejtutocs verausgabten Sintur= foîten $=\mathrm{c}$.

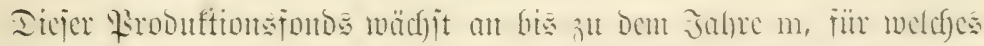

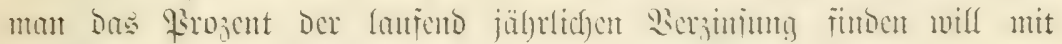
p Prozent, oem angenommenen falfulationsprozente, auj

$$
(B+V+c) 1,0 p^{m} \text {. }
$$

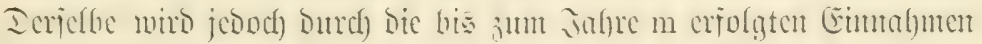

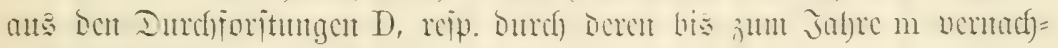
werteten Beträgen entlajtet.

Dian erfält io Den entlajteten \$roduttionsaupwand $=$

$$
(B+V+c) 1,0 p^{m}-\left(D_{a} 1,0 p^{m-a}+D_{b} 1,0 p^{m-b}+\right)
$$

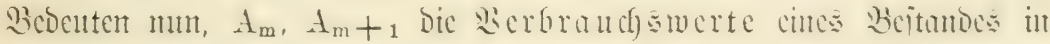
Den Salyren $m,(m+1)$, io ijt $A_{m+1}-A_{m}$ bic vom salre $m$ bis zum

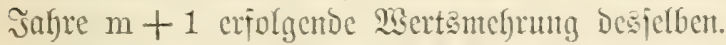

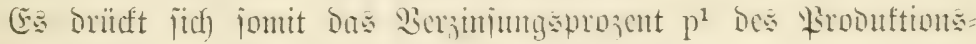
fond: zน Ende Des Jafres $m$ Durd die Formel nus:

$$
p^{1}=\frac{\left(A_{m+1}-A_{m}\right) 100}{\left.\left.B+r+c) 1,() p^{m}-\left(D_{a} 1,0\right)^{m-a}+D_{b}, 1,1\right) p^{m-b}+\cdots *\right)}
$$

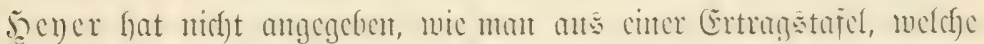

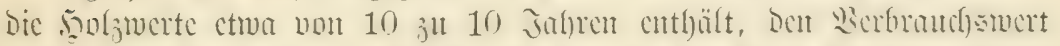

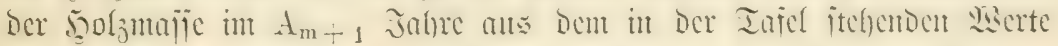

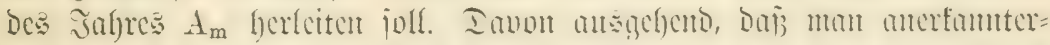

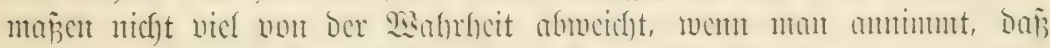

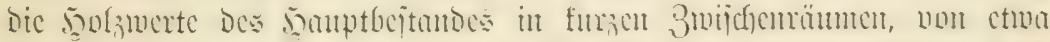

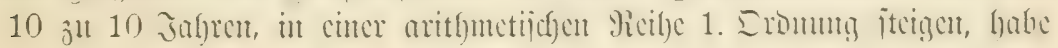

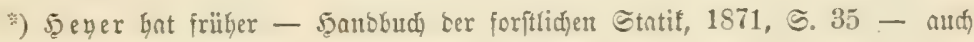
jorgende Räherungsiormel gegeben:

$$
p^{1}=\frac{(A m+1-A m) 100}{A m+B+V}
$$

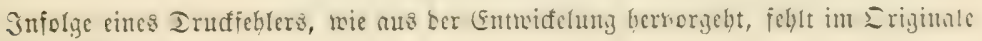
ber Faltor 100, bes 3äblers. 


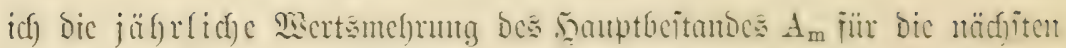
10 Ial)re $A_{m}+10$ ausgebrïct ourd) bic Formel

$$
\underline{A_{\mathrm{m}}+10}-A_{\mathrm{m}}
$$

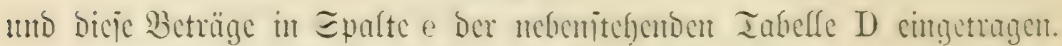
(Siefe die Tabelle D auf ङ. 25.)

Dic Eummen ber von Begrïndung bes Bcitandes an criolgten unt

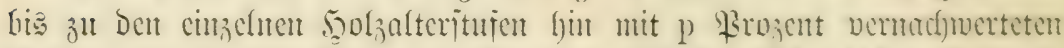

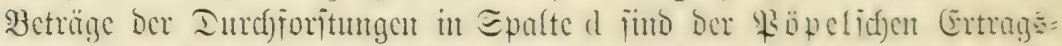

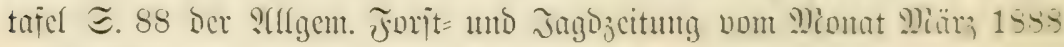
cntnommen.

Iic Epalte f cuthült bic Beträge $\left(B+T^{-}+c\right) 1,(1) p^{\mathrm{m}}$ umb bic Emalte er

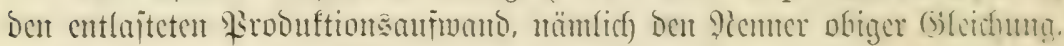
aus weldyer Dam bie 2 eijerprozente Durdy bic Formel

$$
\frac{100 \text { Epalte e }}{\text { Epalte } g}
$$

berechnet uno in Epalte h cingetragen worben fint.

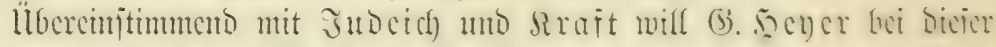

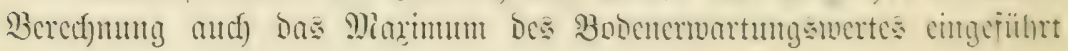
babent.

Der Memer Der Formel $\mathrm{p}^{1}$,

$$
(\mathrm{B}+\mathrm{V}+\mathrm{c}) 1,0 \mathrm{p}^{\mathrm{m}}-\left(\mathrm{D}_{\mathrm{a}} 1,0 \mathrm{p}^{\mathrm{m}-\mathrm{a}}+\mathrm{D}_{\mathrm{b}} 1,0 \mathrm{p}^{\mathrm{m}-\mathrm{b}}+\cdots\right)
$$

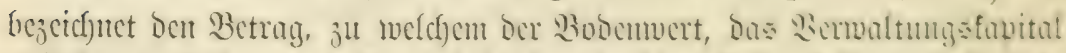

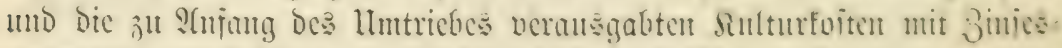

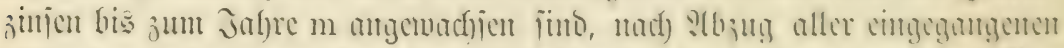

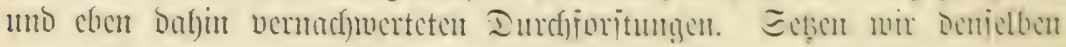
$=\mathrm{N}$, jo hat man bic (3́cidumng

$$
\left.\mathrm{N}=(\mathrm{B}+\mathrm{V}+\mathrm{c}) 1,0 \mathrm{p}^{\mathrm{m}}-\left(\mathrm{D}_{\mathrm{a}} 1,0 \mathrm{p}^{\mathrm{m}-\mathrm{s}}+\mathrm{D}_{\mathrm{b}} 1,0 \mathrm{p}^{\mathrm{m}-\mathrm{b}}\right)+\cdots\right)
$$

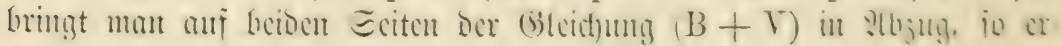
bält maแ :

$\left.\mathrm{X}-(\mathrm{B}+\mathrm{T})=(\mathrm{B}+\mathrm{T}) 1,0 \mathrm{p}^{\mathrm{m}}+\mathrm{c} \cdot 1,0 \mathrm{p}^{\mathrm{m}}-(\mathrm{D})_{3} 1,0 \mathrm{p}^{\mathrm{m}-\mathrm{a}}+\cdots\right)-(\mathrm{B}-\mathrm{Y})$ $\mathrm{N}-(\mathrm{B}+\mathrm{V})=(\mathrm{B}+\mathrm{V})\left(1,0 \mathrm{p}^{\mathrm{m}}-1\right)+\mathrm{c} .1,0 \mathrm{p}^{\mathrm{m}}--\left(\mathrm{D}_{\mathrm{a}} 1,0 \mathrm{p}^{\mathrm{m}-\mathrm{a}}+\cdots\right)$

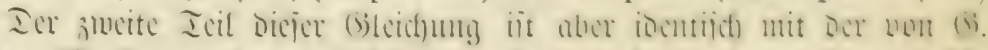

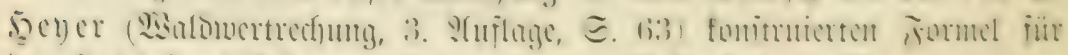

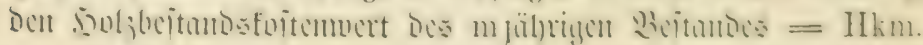

Dallect

$$
\begin{aligned}
& \mathrm{N}-(\mathrm{B}+\mathrm{V})=\mathrm{Hkm} . \\
& \mathrm{N}=(\mathrm{B}+\mathrm{V})+\mathrm{Hkm} .
\end{aligned}
$$

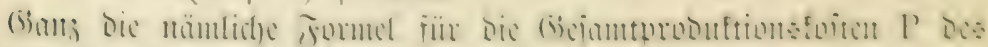

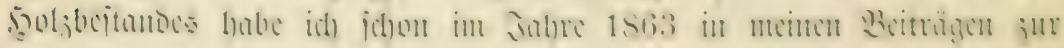




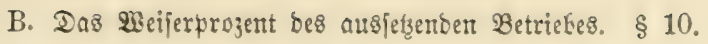

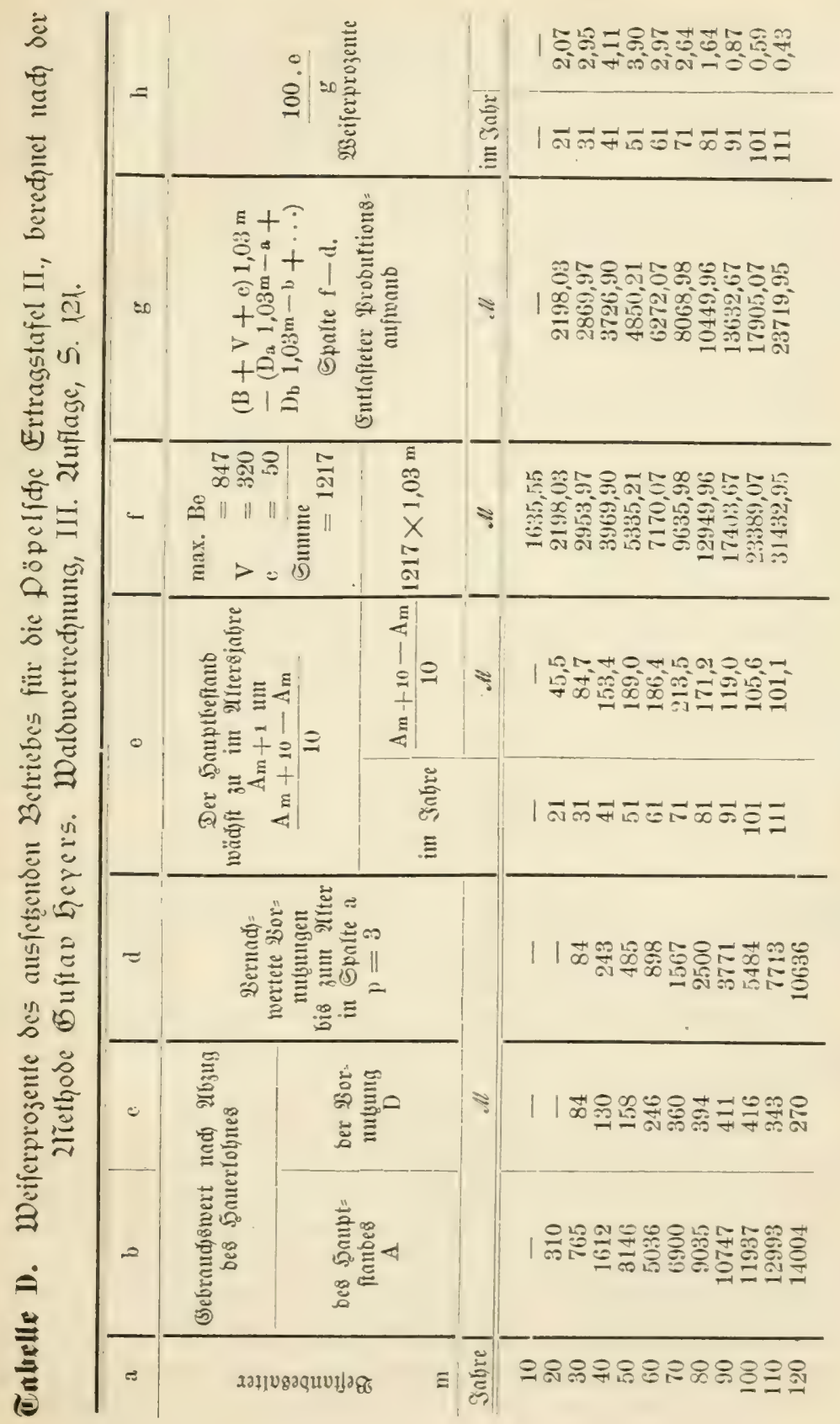




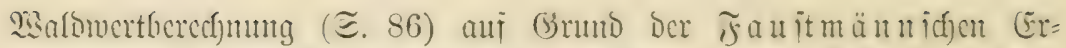
B̈rterungen iffer bejen (jegenjtand in ber Formel 13 a gegeben, meld)e mux

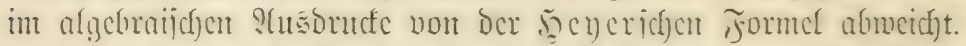

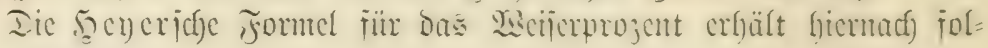
gento (Sejtalt:

$$
\mathrm{p}^{1}=\frac{\mathrm{A}_{\mathrm{m}+1}-\mathrm{A}_{\mathrm{m}}}{\mathrm{B}+\mathrm{V}+\mathrm{Hk}_{\mathrm{m}}} .
$$

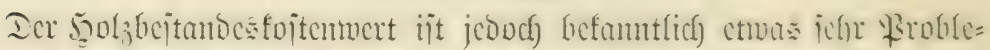

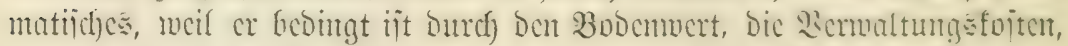

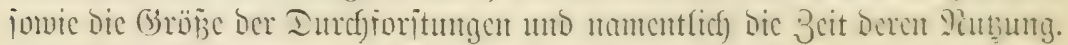

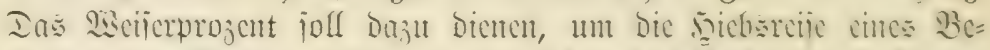

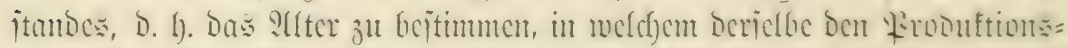

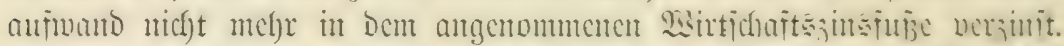

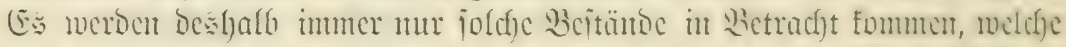

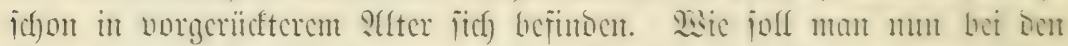

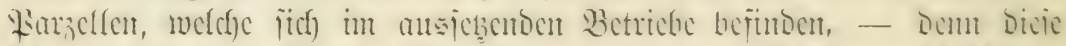

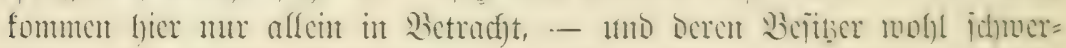

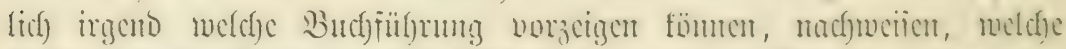

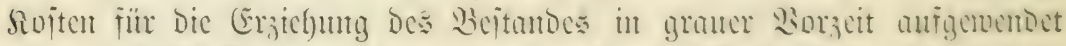

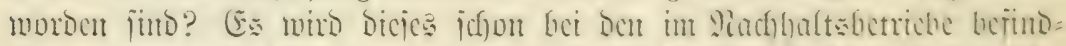

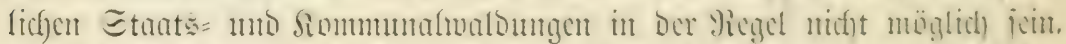

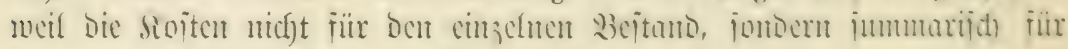
Dic ganze Betriebstajic aufgciüfrt werden.

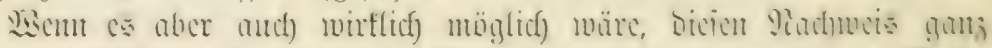

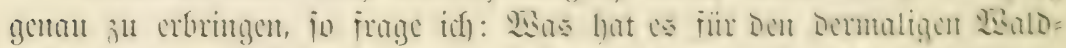

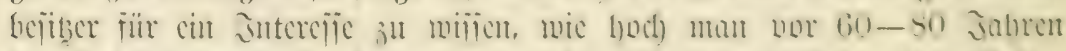

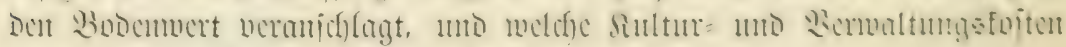
man zu jencr Bcit veranggabt hat?

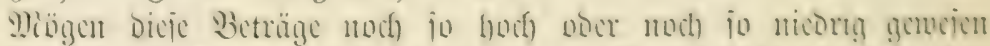

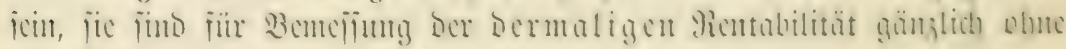
alle Bebeutumg.

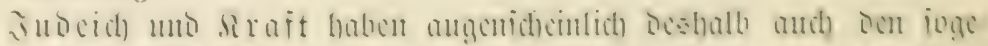

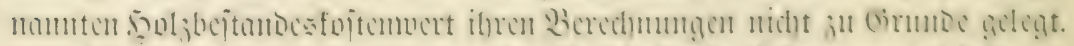

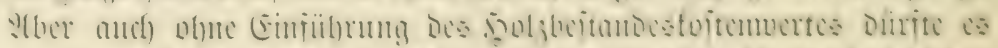

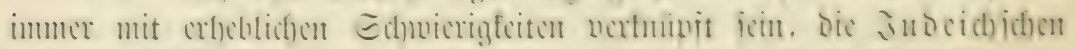

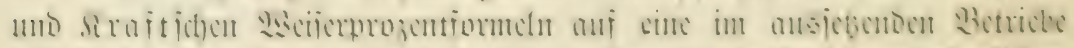

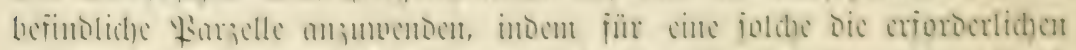

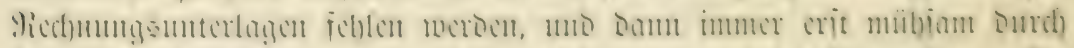

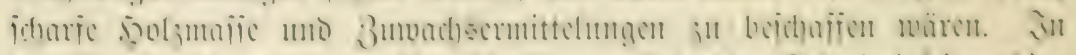

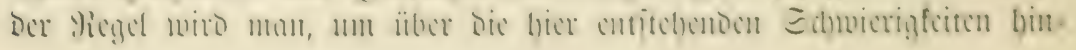




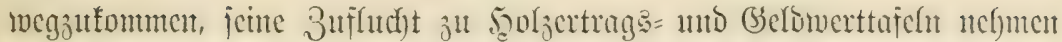

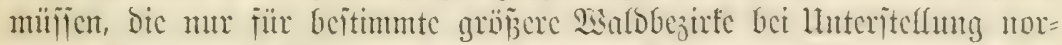

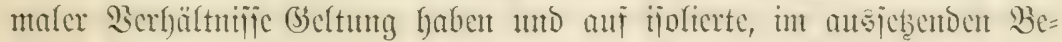

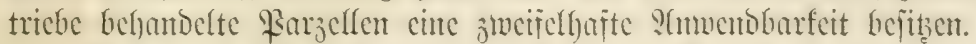

Stber gerabe it jolden, im nadybaltigen Betriebe befüblichen Wal=

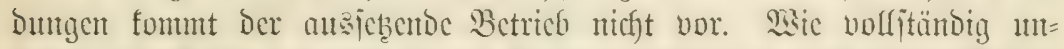

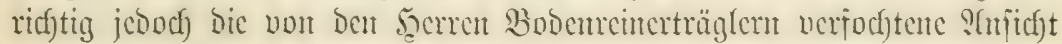
iit, baf bie fïr ijolicrte

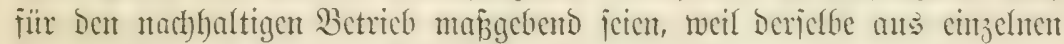

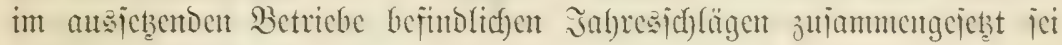
- merde ich fpäter nacfjweijen.

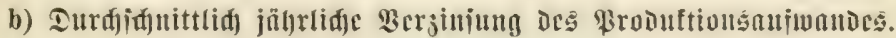

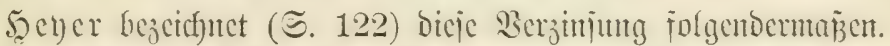

Iic laufende Seroinjung ijt cine unglcidymänige. 2isill man bie

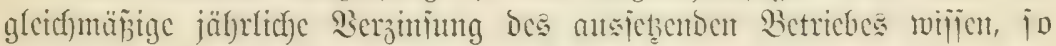
verwandel man die innerfalb ber llmtricbseit criolgenden

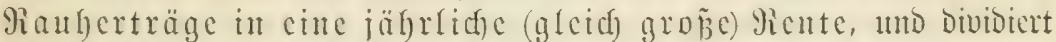
Diejelbe Durch Das Sapital Der ßrobuftionstojten.

Multiplijiert man ben gewomenen Euntentent mit 100, jo crljält man

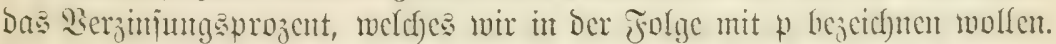

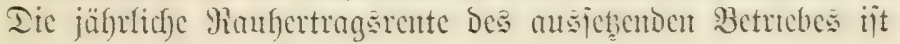

$$
=\frac{\left(A_{u}+D_{a} 1,0 p^{u-a}+\ldots D_{q} 1,0 p^{u-q}\right) 0,0 p}{1,0 p^{u}-1}
$$

Das Rroouftiontafapital i

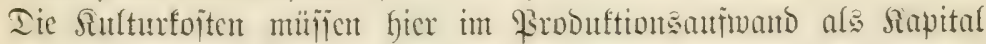

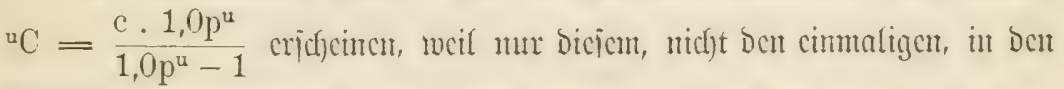

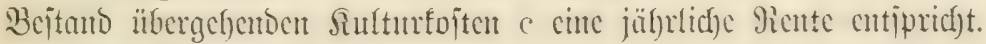

Der $\mathfrak{A}$ (usbrutef in obiger Formel:

$$
\frac{A u+D_{a} 1,0 p^{u-a}+\ldots \ldots D_{q} \cdot 1,0 p^{u-q}}{1,0 p^{u}-1}
$$

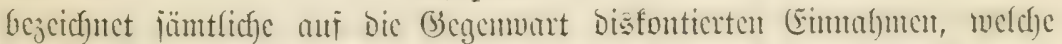

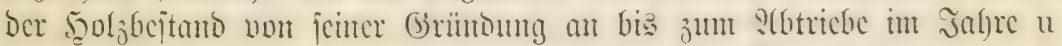

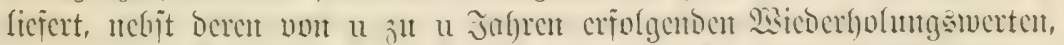

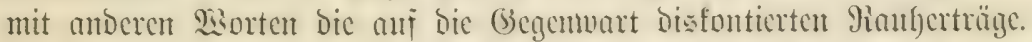

Daß gegentwärtige - (D. b. Das a gered ncte, in wirflichecit jedoch nidft vorfandenc, Boje) Pro= Duftionsfapital ịt $=\mathrm{B}+\mathrm{V}+\frac{\mathrm{c} \cdot 1,0 \mathrm{p}^{\mathrm{u}}}{1,0 \mathrm{p}^{\mathrm{u}}-1}$. 


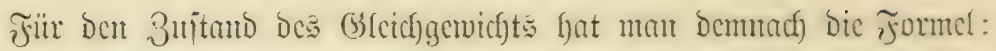
$\frac{\mathrm{Au}^{\mathrm{u}}+\mathrm{Da} \cdot 1,0 \mathrm{p}^{\mathrm{u}-\mathrm{a}}+\ldots \cdot \mathrm{Dq} \cdot 1,0 \mathrm{p}^{\mathrm{u}-\mathrm{q}}}{1,0 \mathrm{p}^{\mathrm{u}}-1}=\mathrm{B}+\mathrm{V}+\frac{\mathrm{c} \cdot 1,0 \mathrm{p}^{\mathrm{u}}}{1,0 \mathrm{p}^{\mathrm{u}}-1}$

Sicrans folgt:

$$
\mathrm{B}=\frac{\mathrm{Au}^{\mathrm{u}}+\mathrm{Da} \cdot 1,0 \mathrm{p}^{\mathrm{u}-\mathrm{a}}+\ldots \cdot \mathrm{Dq} \cdot 1,0 \mathrm{p}^{\mathrm{u}-\mathrm{q}}-\mathrm{c} \cdot 1,0 \mathrm{p}^{\mathrm{u}}}{1,0 \mathrm{p}^{\mathrm{u}}-1}-\mathrm{V} \text {. }
$$

Eื Die Umtriebszzeit $u$.

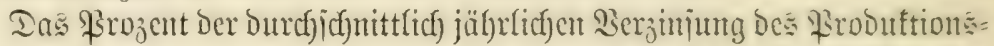

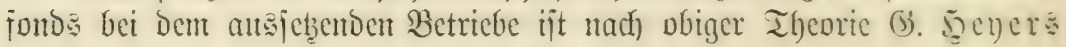

voer

$$
\left.p=\frac{\left(\frac{A u+D a}{D} \cdot 1,0 p^{u-a}+\ldots . D D^{a} \cdot 1,0 p^{u-q}\right.}{1,0 p^{u}-1}\right) 0,0 p \cdot 100
$$

$$
p=\frac{\left(\frac{\mathrm{Au}+\mathrm{Da} \cdot 1,0 \mathrm{p}^{\mathrm{u}-\mathrm{a}}+\ldots \cdot \mathrm{Dq} \cdot 1,0 \mathrm{p}^{\mathrm{u}-\mathrm{q}}}{1,0 \mathrm{p}^{\mathrm{u}}-1}\right) \mathrm{p}}{\mathrm{B}+\mathrm{V}+{ }^{\mathrm{u}} \mathrm{C}}
$$

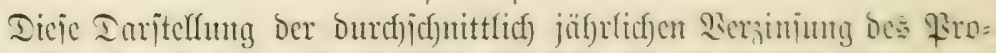

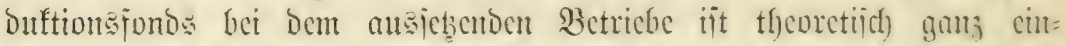

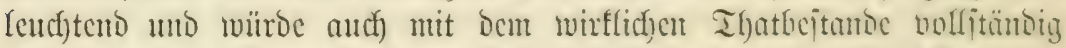

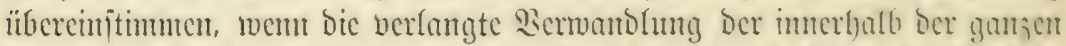

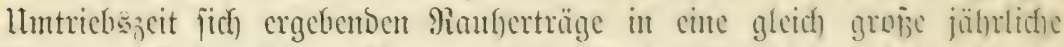

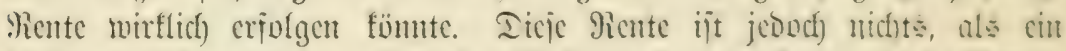

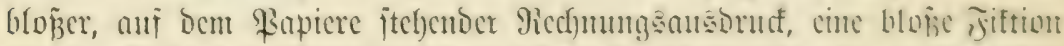

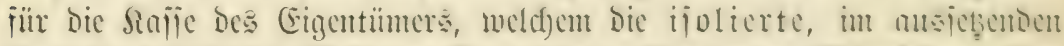

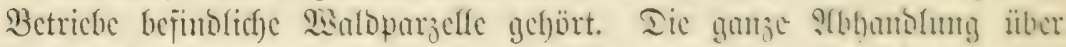

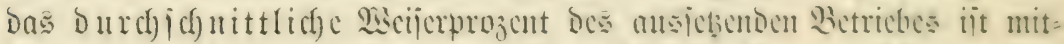

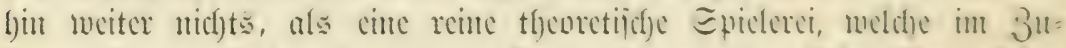

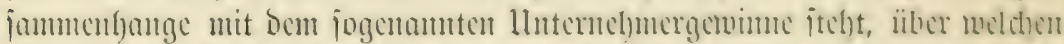

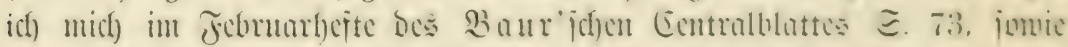

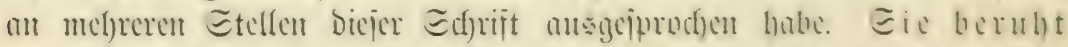
auf ber vou mir mieberholt als unriditig besechneten :In

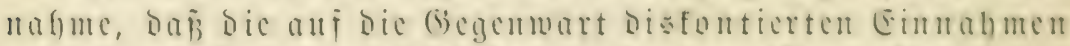

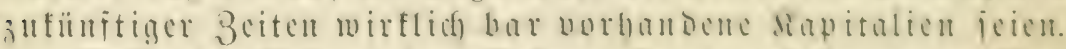

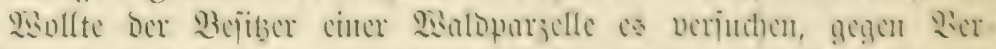

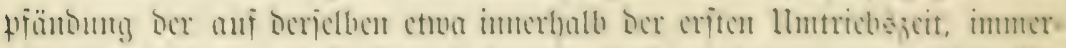

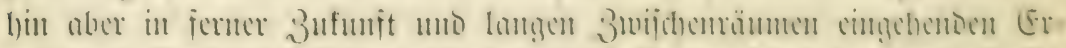

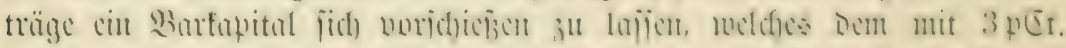

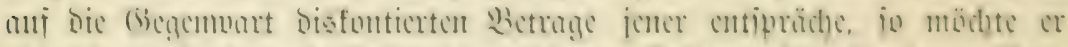

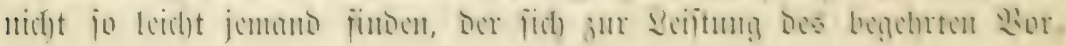




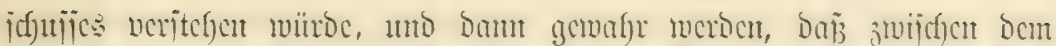

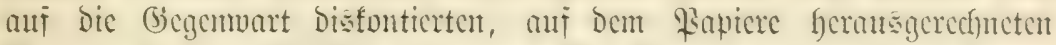
2iserte jufinitiger Erträge uno cincm Bar=Siapital cin gewaftiger llnter=

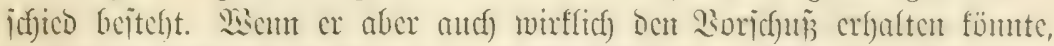

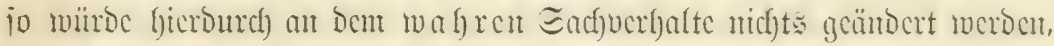

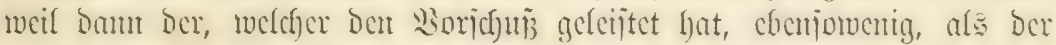
critere cine jüf)rfiche Rente aus bem $\mathfrak{B} a$ aldo hezichen fumt.

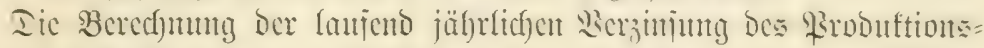

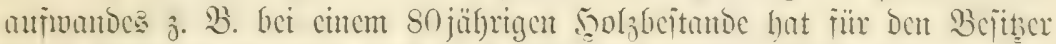

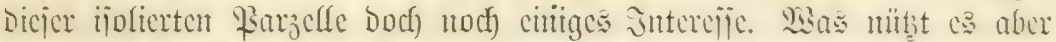

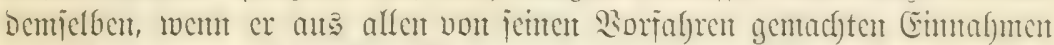

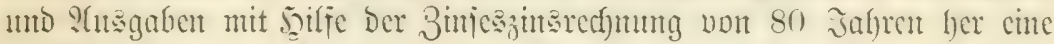

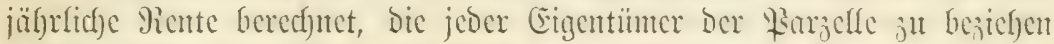

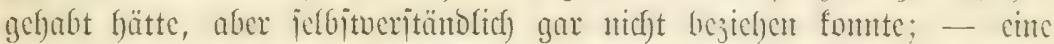

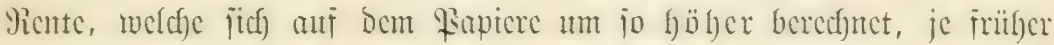

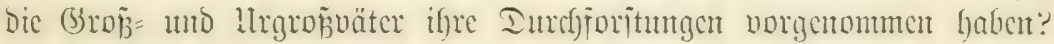

\section{$\$ 11$.}

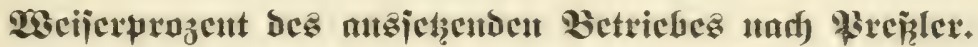

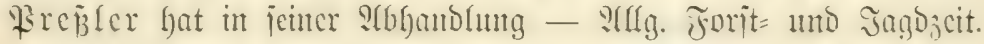
non $1860, \Xi .188$ ரि. - cincu allgemeiten algebratichen S(usoruct für

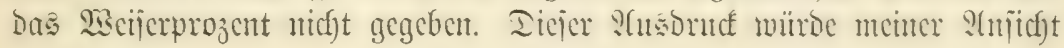
nach) lauten:

$$
W=\frac{\left(H_{m}+1-H_{m}\right) 100}{H_{m}+G}
$$

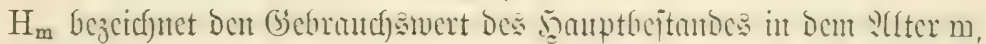
jür welchen Das 2 seijerprozent ermittelt werbent joll.

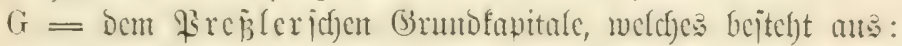

a) Dem Mar. "Be für bie llmtrieb引zeit u,

b) Dem Berwaltungafapitale $\frac{\mathrm{v}}{0,0 \mathrm{p}}=\mathrm{V}$,

c) Dem Situlturfapitale.

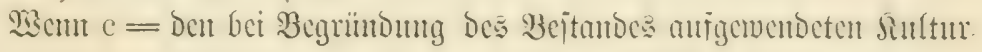
tojten, jo ijt bas Sulturfapital $={ }^{u} \mathrm{C}=\frac{\mathrm{c} \cdot 1,0 \mathrm{p}^{\mathrm{u}}}{1,0 \mathrm{p}^{\mathrm{u}}-1}$.

Die Formel roübe beshalb lauten:

$$
W=\frac{\left(H_{m+1}-H_{m}\right) 100}{H_{m}+V+{ }^{u} \mathrm{C}+{ }^{u} B e} .
$$

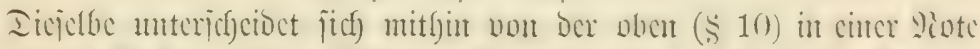

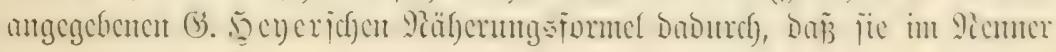




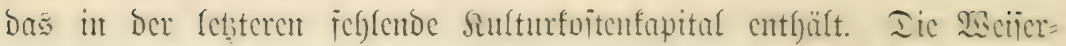
prozente werden beslyalb etwas flemer ausjallen.

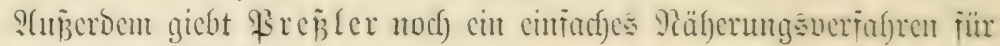

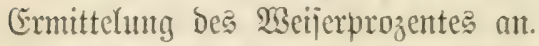

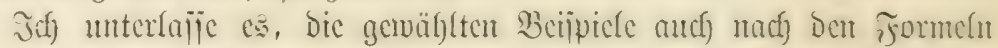

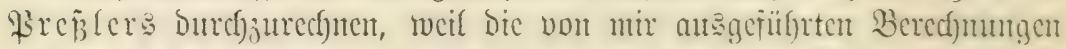

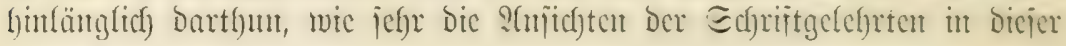
Sache auscinander gehen.

\section{\$ 12 .}

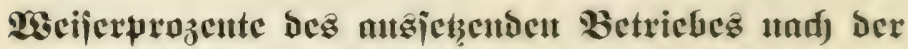

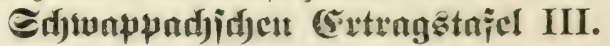

(Siche bie Tabelle E auj ऽ. 31.)

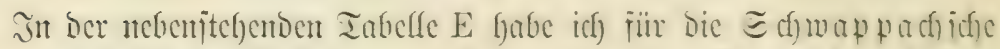

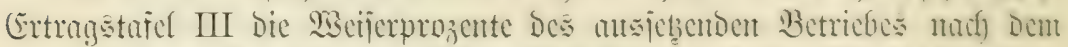

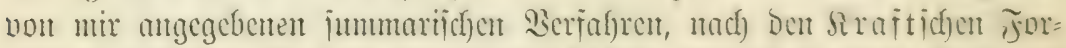

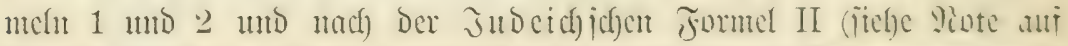

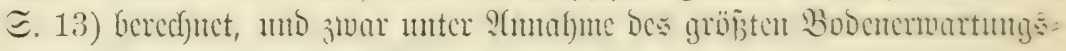
wertes.

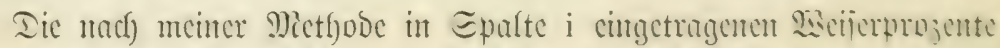

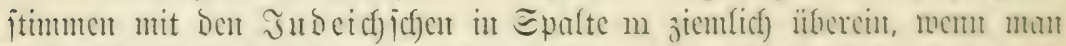

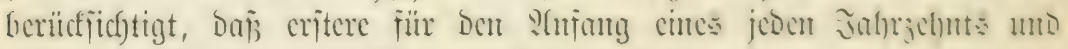
leţtere ungefälyr für bie Mitte Deşelben gelten.

\section{\$ 13.}

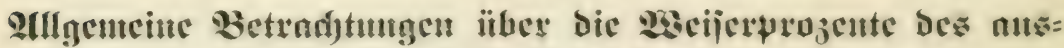 jetzenton Betricbes.}

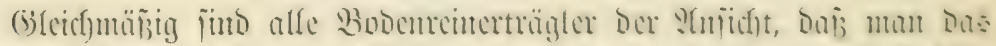

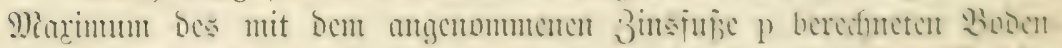

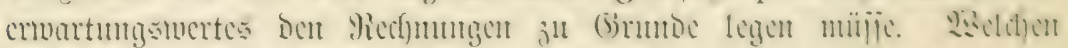

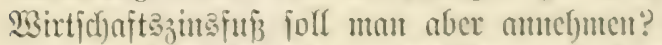

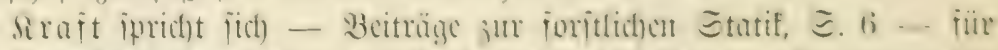

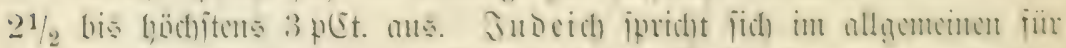

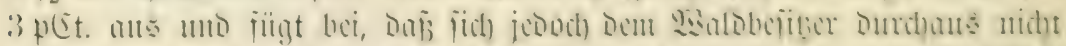

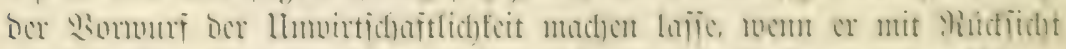

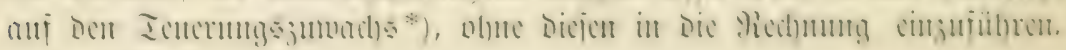

*) Diefer Teucrumgsumads, Deffen \&orats \& Sabre, gefducige für ciue ganje llutriebsjeit cin Diug ber Unubiglid.

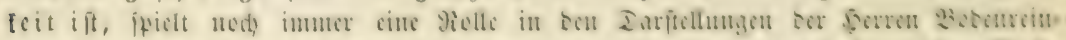

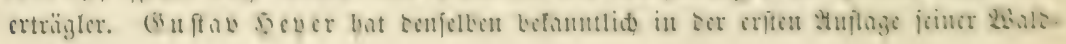




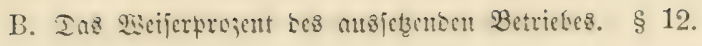

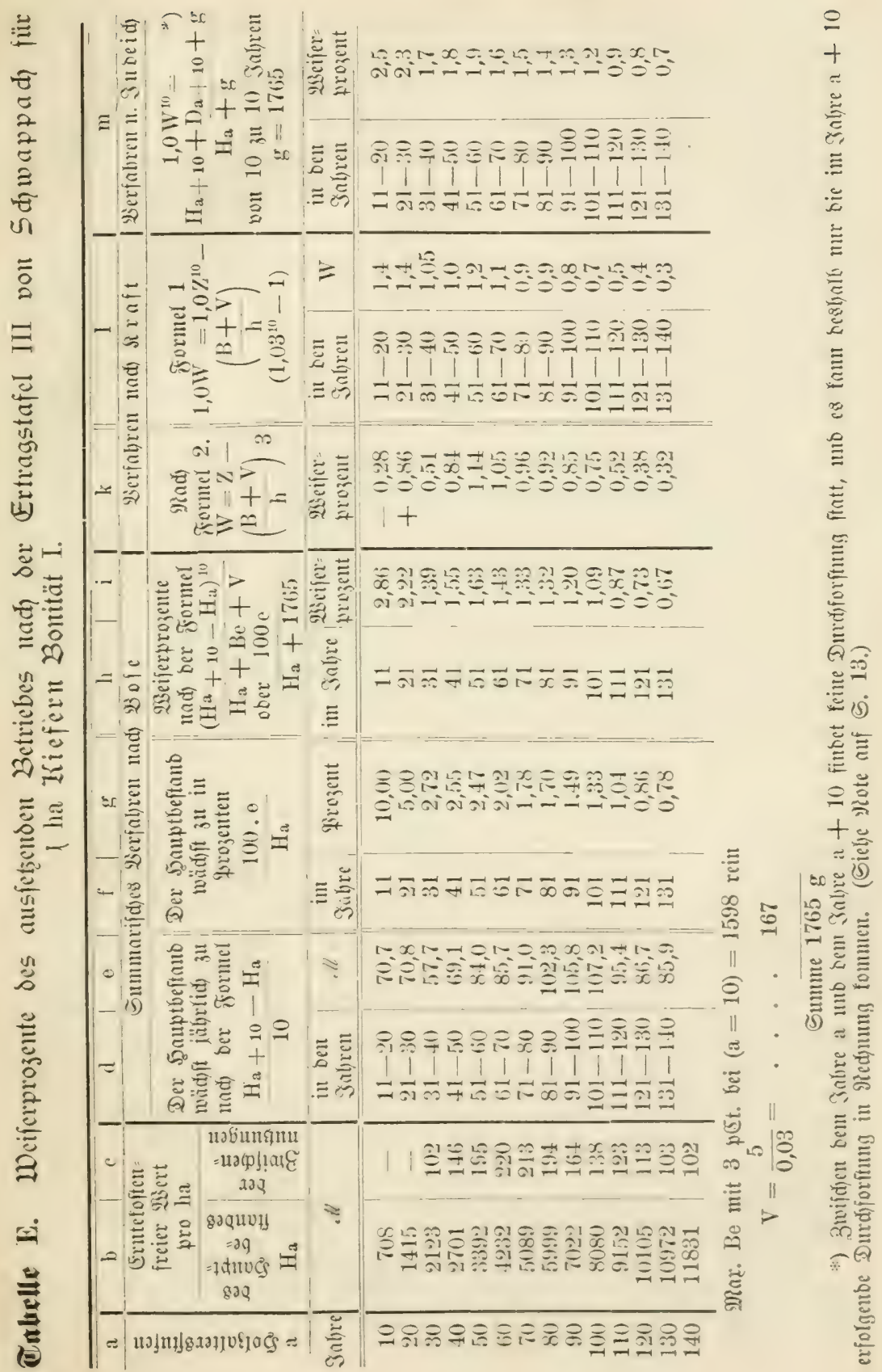




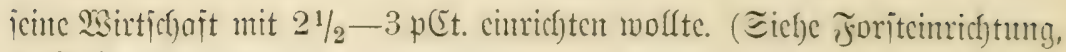
4. 2(ufl., ธ. 71.)

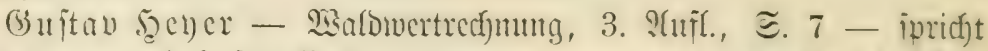

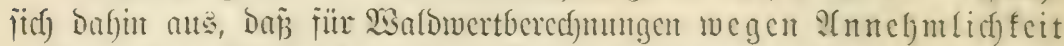

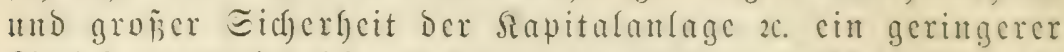

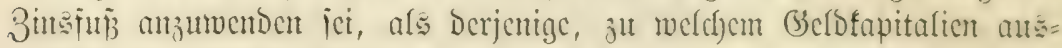
gelieben zu werden pflegent.

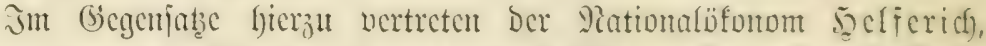

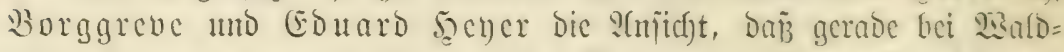

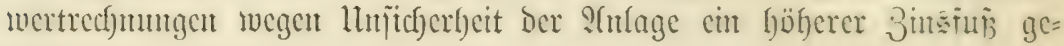
rechtiertigt iei.

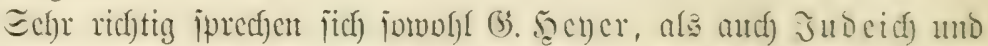

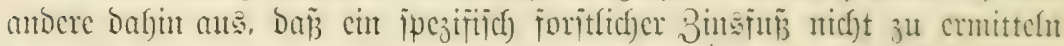

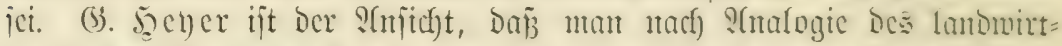

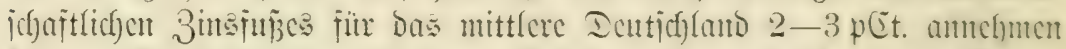

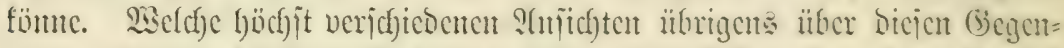

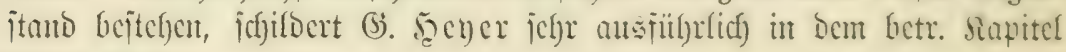
jeines $\mathfrak{B u d f e}$.

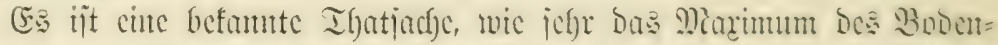

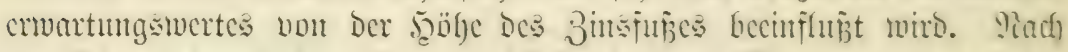

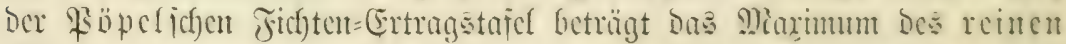
Bobenerwartumģwertes (crift. V)

$$
\begin{gathered}
\text { bei } 3 \text { pCet. } 847 \text {.ll } \\
\text { " } 2 \text { " } 2304 \text { " }
\end{gathered}
$$

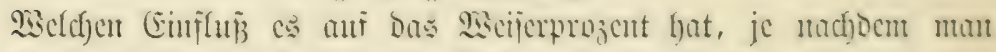

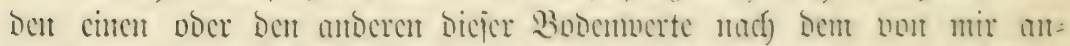

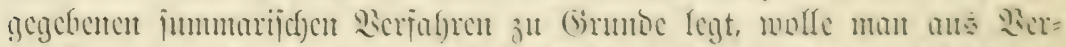

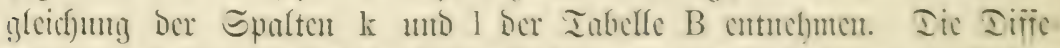

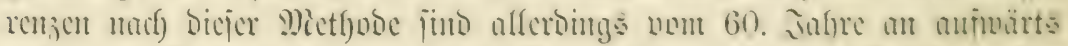

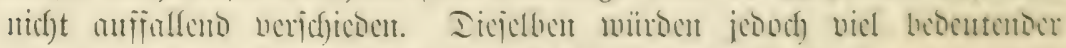

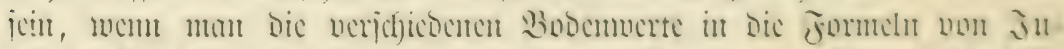

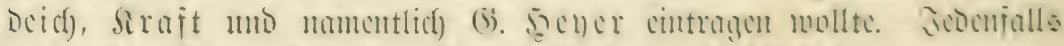

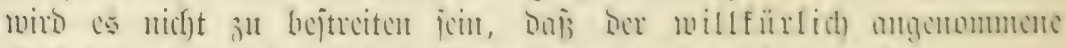

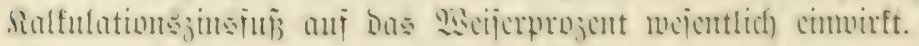

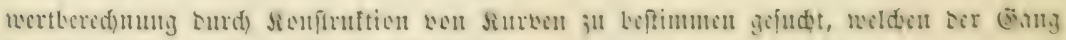
Der Deolipreife jebod burdanas midut folgte.

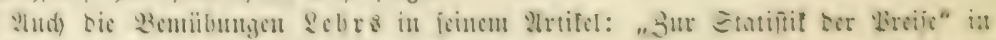

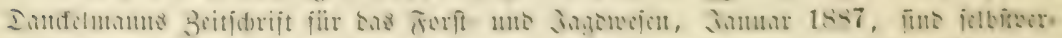

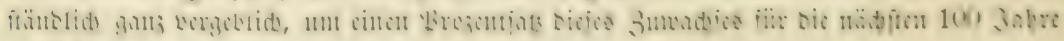
\$it Eeftimumen. 


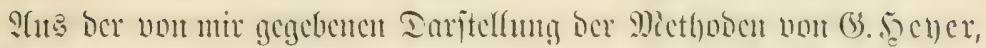

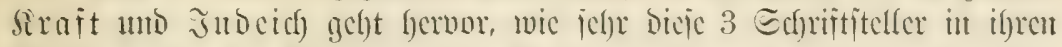

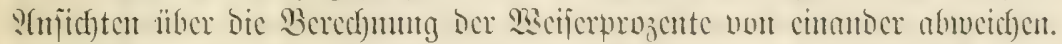

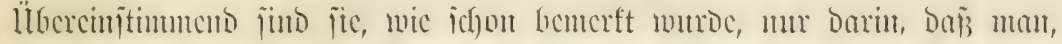

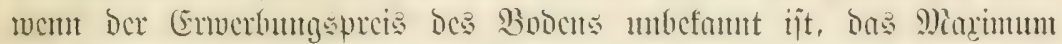

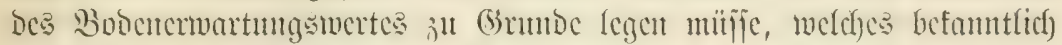

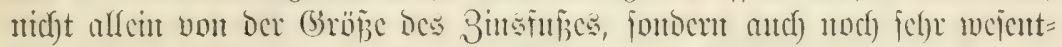

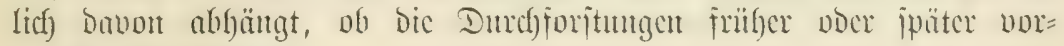
gentommen toerden.

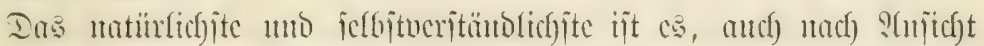

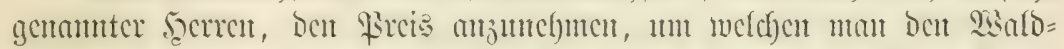

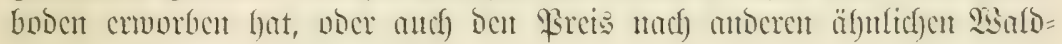

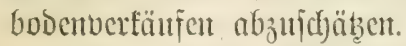

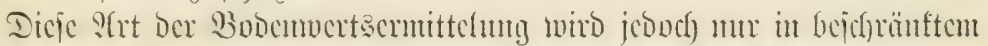

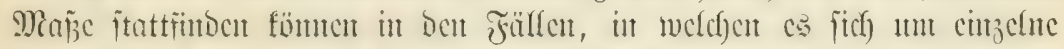

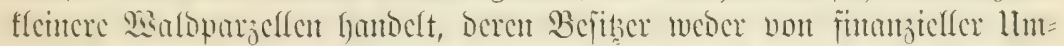
trichascit, noch von 2iscijerprojent je ctwas gebört haben. Piur bci

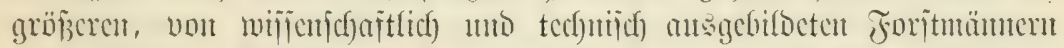

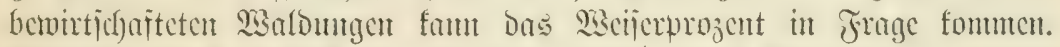
E. wird jedoch wolf faum jemantom cinfalfen, Dest Bobentuert berartiger

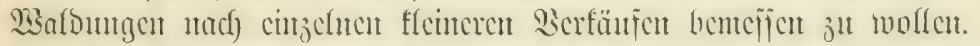

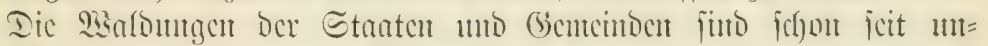

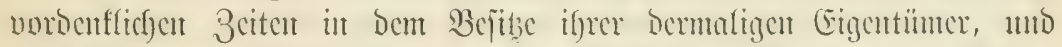

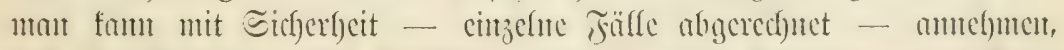

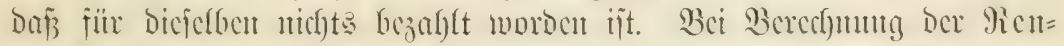

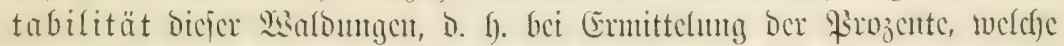

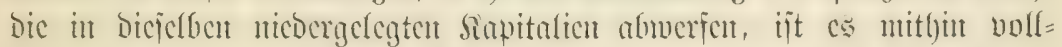

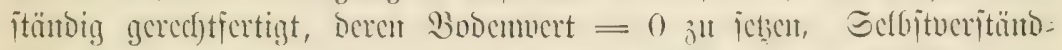

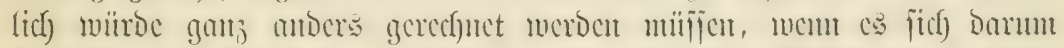

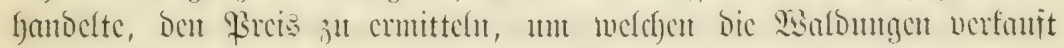
merben folfen, oder viclmelgr verfant meroen fömen.

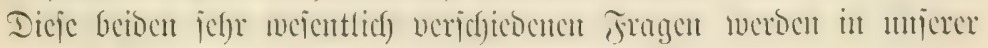
jogenamten Waldwertred)mutg aber immer verquicft.

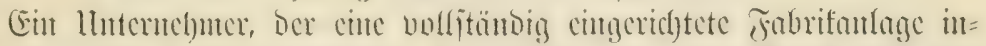

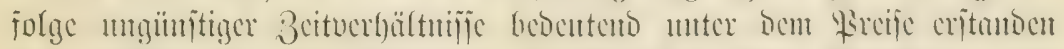

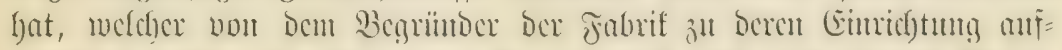

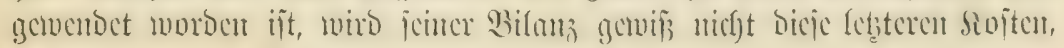
jondern nur Den Afufoupepreis zu Brunde legen.

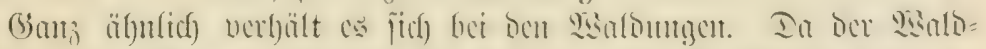

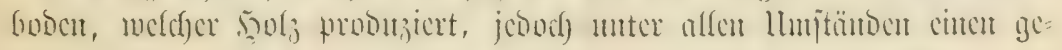
Boie, Dab foritlide Weiferprojent. 


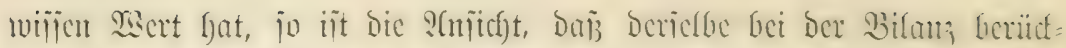

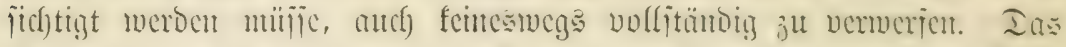

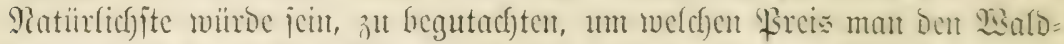

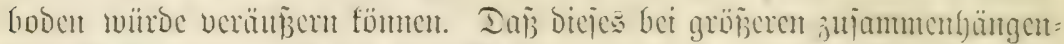

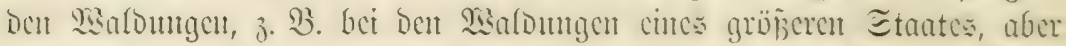

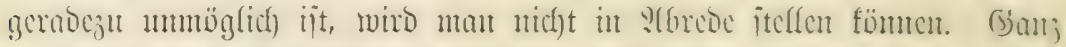

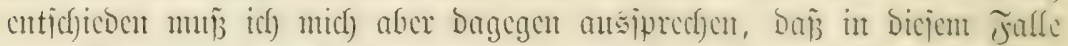

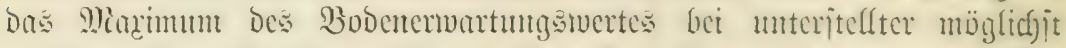

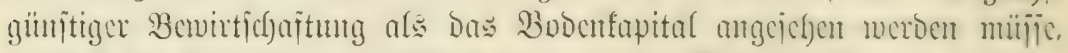

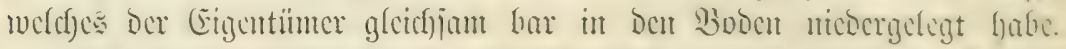

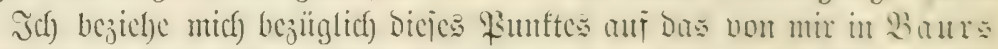

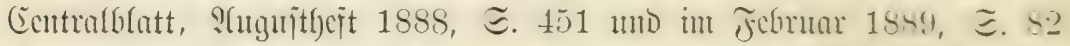

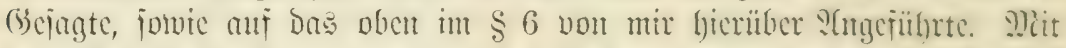

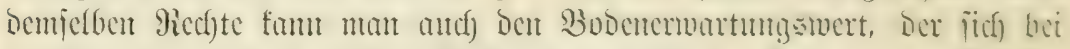

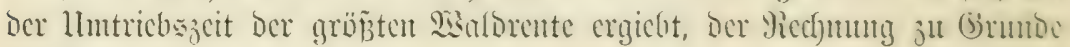
legen.

2iseder Der cinc, noch ber andore 2isert fam einem Bartupitali

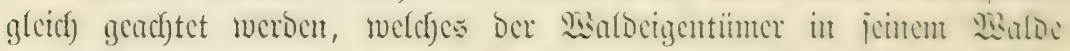
nicocrgelegt bat.

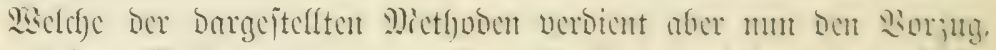

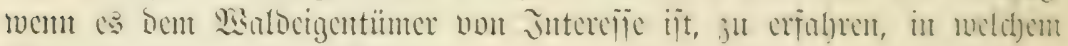
Frozente cin cinzeluer Bejtand fortwäclyjt.

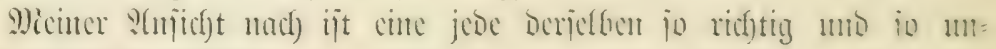
rid)tig, wie bic andere.

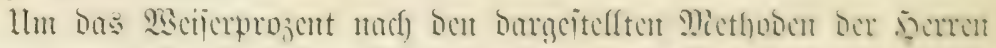

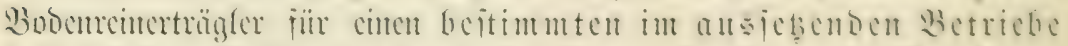

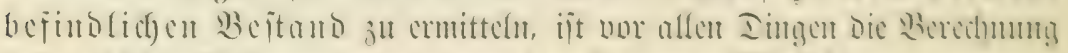

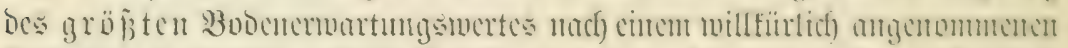

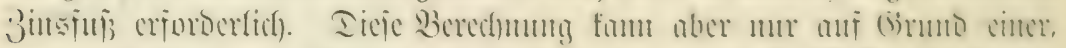

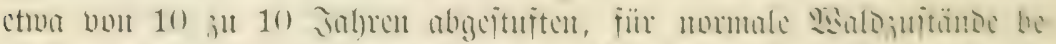

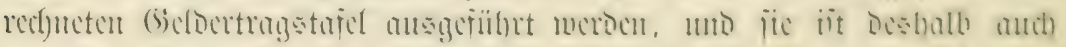

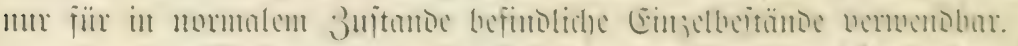

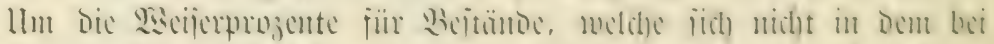

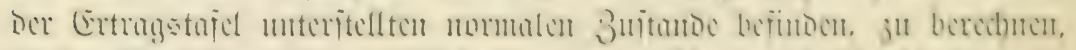

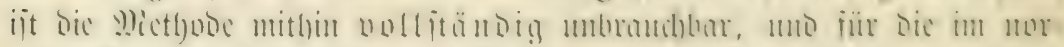

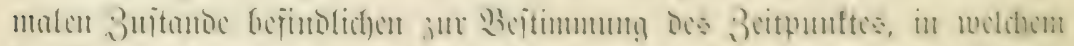

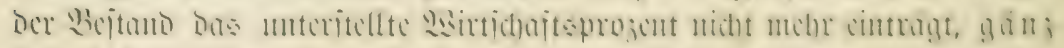

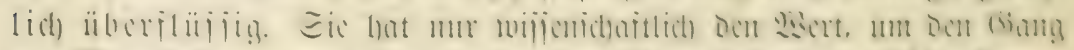

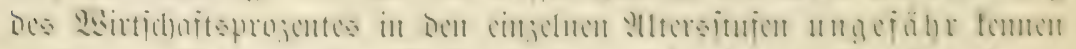

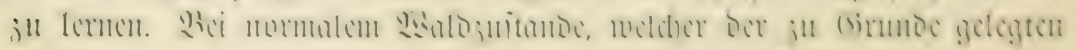




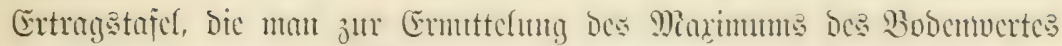

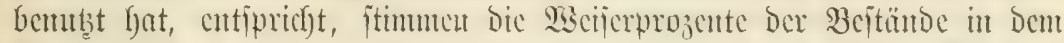

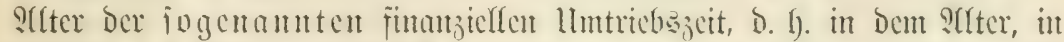

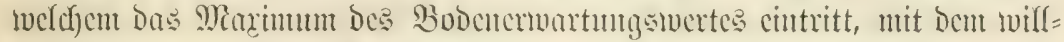

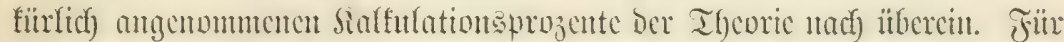

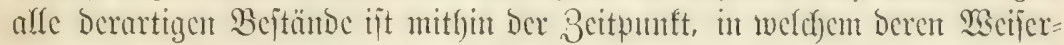

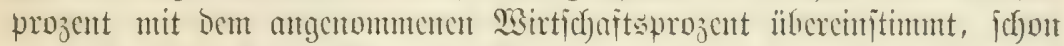

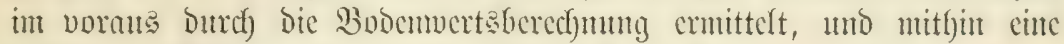

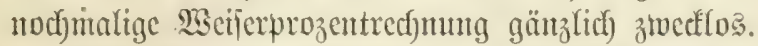

Bei abunormen Beftünden ift bic Bcitimmung des Dermaligen (Sic=

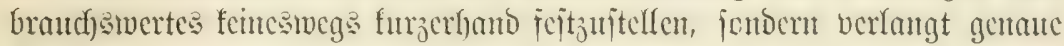

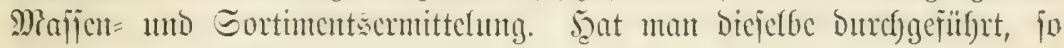
tritt bic weitere Frage Geran:

Ilm welefen Betrag wiro Der betreffende Bejtant it Den nädjiten n (10) ฐaf)rett zutwachjen?

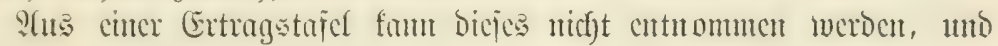

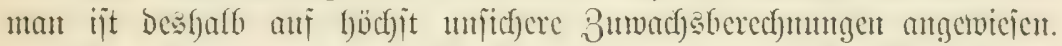

Die Weijerprojente für Dic Ertragstafeln I und II fangen

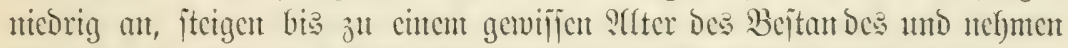

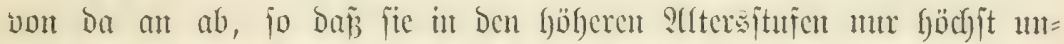

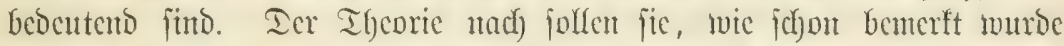

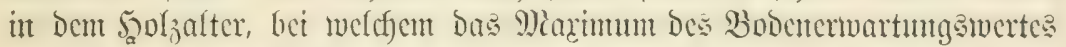
cintritt, mit bem Sialfulationsprojente ïberemptimmen. Es trifft bicice

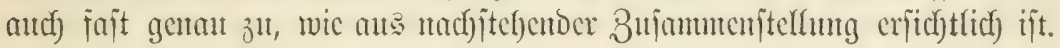

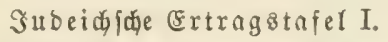

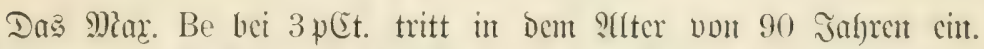

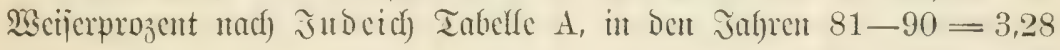

" " " " " " " " $91-100=2,50$

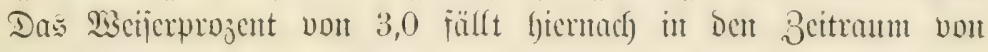
91-100 ฐafren.

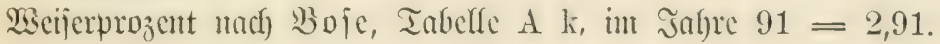

Ertragstafer II bon pöper.

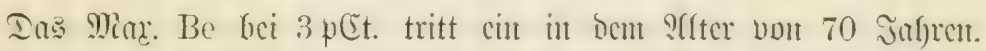

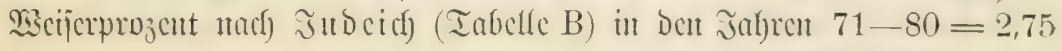

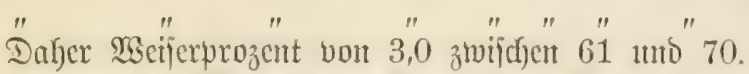

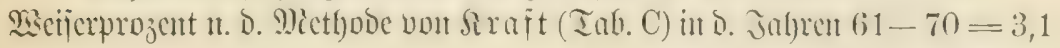

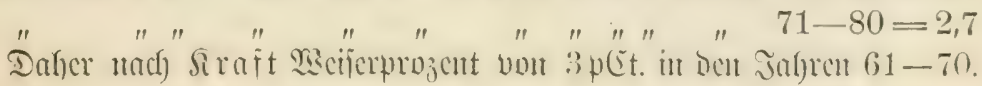




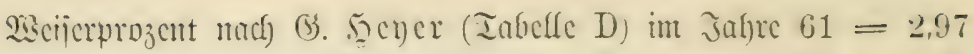
Boje (" B) " " $60=3,00$.

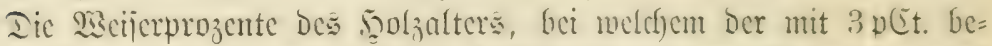

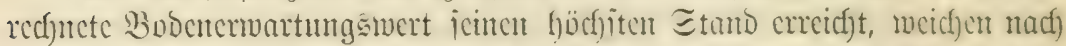
ben voritelgenden Ertragstafeln I mo II io wenig vou 3 pert. ab, baj

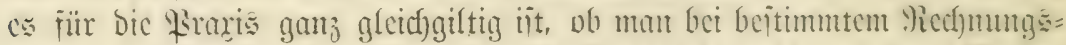
prozent bic jogenannte fintuziclle llmtricbajeit nady bem 2iscijer=

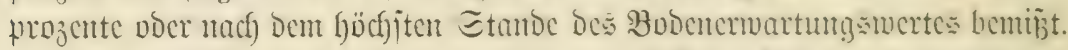

Pantentlich glanbe idf hervorheben ju jollen, Dais bie vout mir ant=

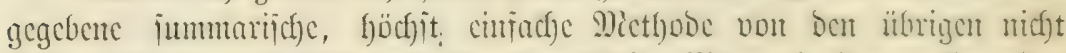

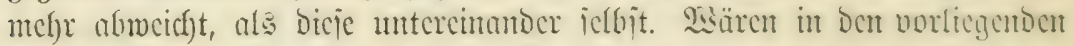

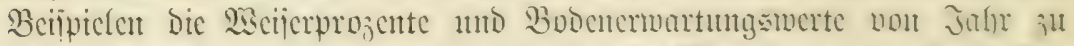

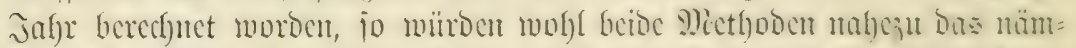

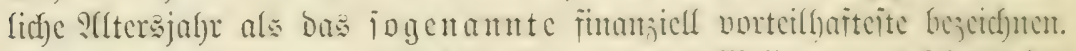

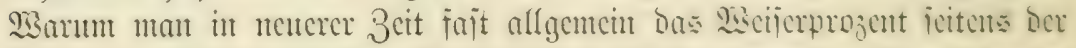

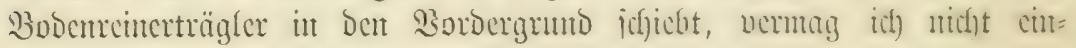
zujebent.

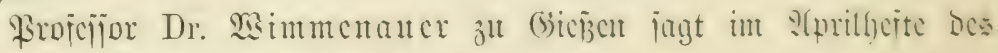
Bauridyen Scutralblatte? von 1889:

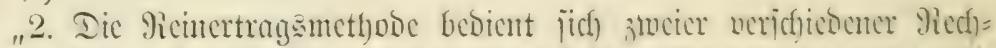

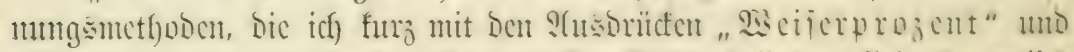

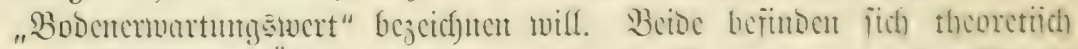

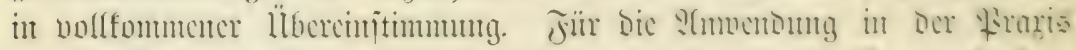
aber cignet jidf itt viclen - mohl son meijten - Fïllen mad) Dem

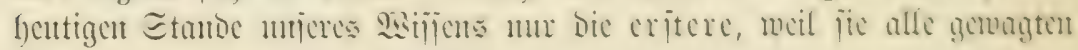
Spefulationen vermciocn läßjt."

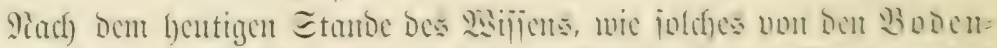

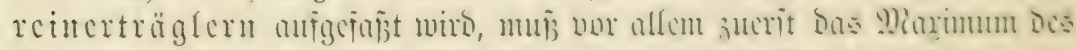

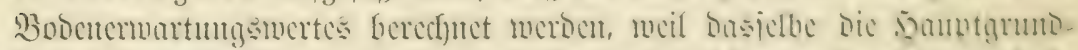

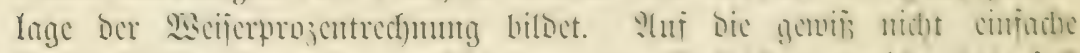

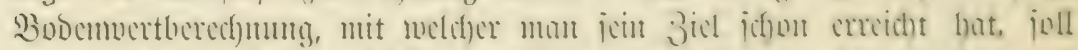

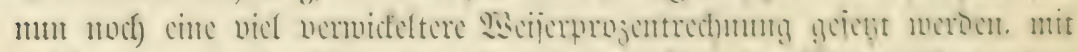

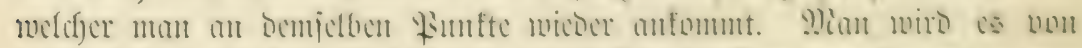

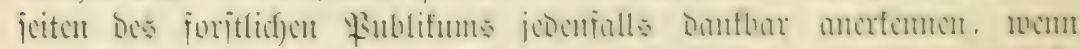

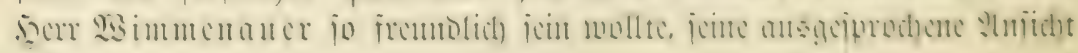
mäl)er âlt motivicren.

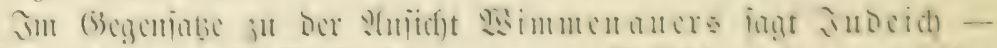

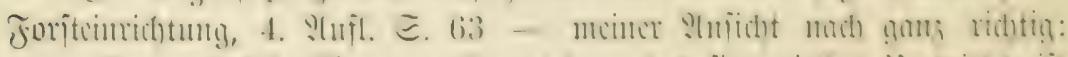

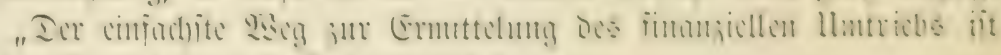
Der Der Berectum de? Bodemuertes." 


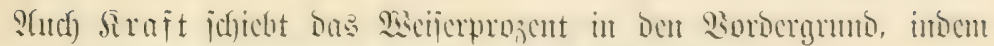
er alif S. 37 iciner Beiträge zur forjtlicfen Statif jagt:

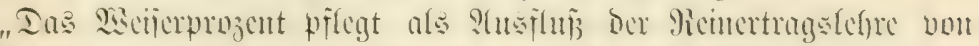

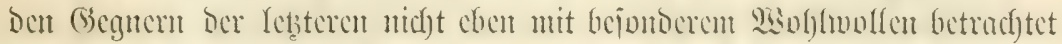

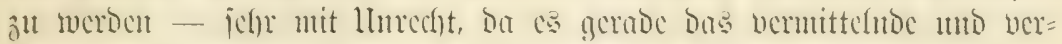

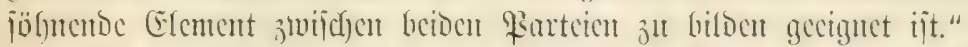

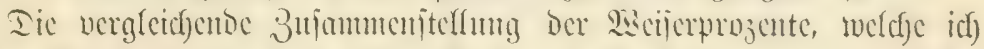

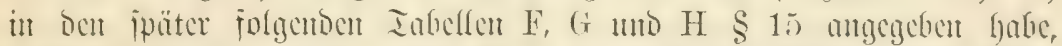

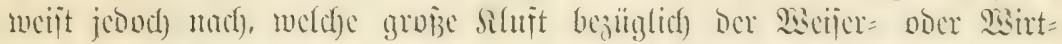

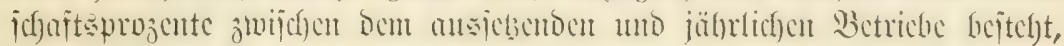

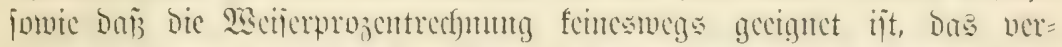

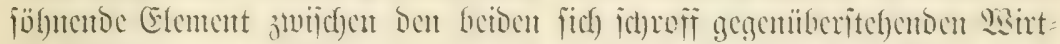

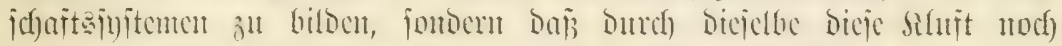

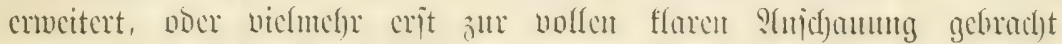
morben ijt.

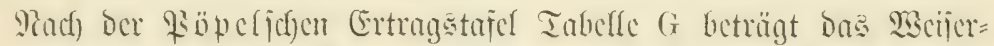

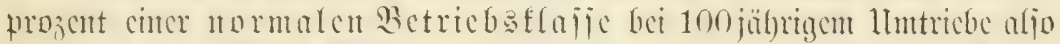

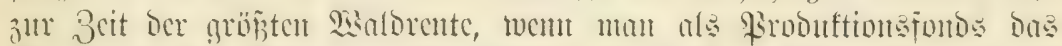

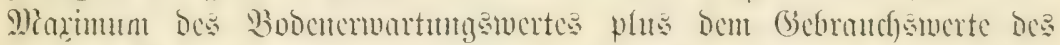

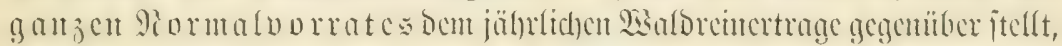

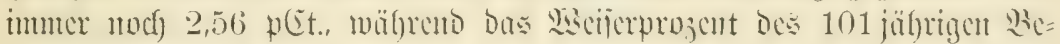
ftondes beträgt:

$$
\begin{aligned}
& \text { nach Soje . . 0,80 pett. } \\
& \text { " 5ecuer. . 0,59 " }
\end{aligned}
$$

Dce 101-110 jäl)rigen Beitandes

$$
\begin{aligned}
& \text { nact) Jubeidy . . 0,85 } \\
& \text { "Siraft . 0,80 }
\end{aligned}
$$

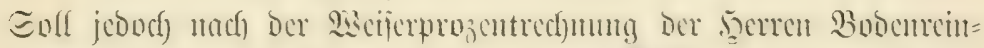

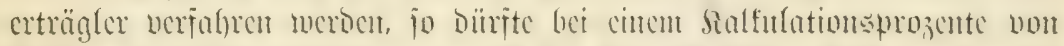

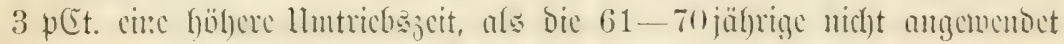
werdoct.

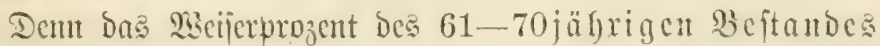

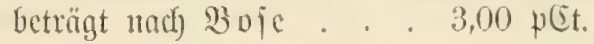

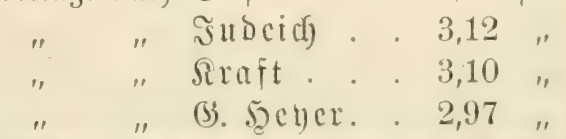

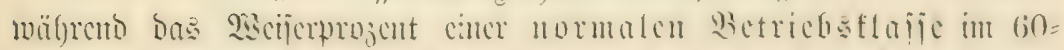

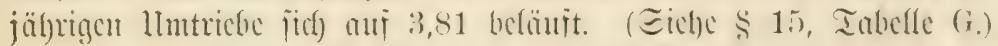

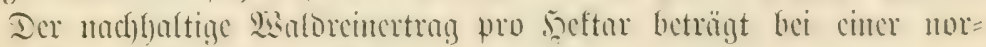
malen Betriebstaife biejer Iajel 


$$
\begin{aligned}
& \text { bei }(\mathrm{u}=100) \quad . \quad \text {. 131,3 } \\
& \text { " }(u=70) . .102,2 \text {. . } \\
& \text { " eriterer melyr. . } 29,1 \%
\end{aligned}
$$

Dafer jäfrrfiçer Mefrertrag Der eriteren

$$
\text { Gci } 10000 \text { ha }=10000 \times 29,1=291000 \% \text {. }
$$

Der reine Bodenerwartungsmert beträgt pro Seftar

$$
\begin{aligned}
& \text { bei }(\mathrm{u}=70)={ }^{\mathrm{B}} \mathrm{Be}=847,0 \text { 川 } \\
& \text { bci }(\mathrm{u}=100)={ }^{\mathrm{B}} \mathrm{B}=583,0 \text { " }
\end{aligned}
$$

("Be- " $\mathrm{B}=$ ) bet criterem mefyr $=264,0$ "ll

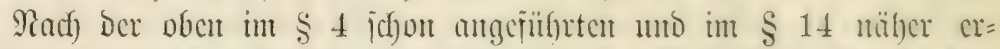

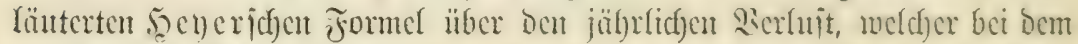

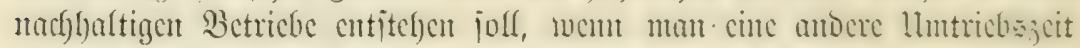
als bie bes gröpten Bobchemuartungswertes it STmentung bringt, be=

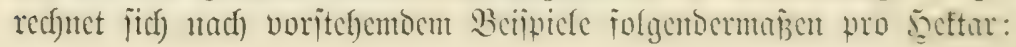

$$
\begin{gathered}
\frac{\left({ }^{\mathrm{B} B}-{ }^{\mathrm{u} B}\right)(1,03100-1)}{100} \\
\frac{264 \times 18,2186}{100}=48,0971 \text {./ pro jeftar. }
\end{gathered}
$$

Daher für 10000 ha auf $10000 \times 48,0971=480971$ All,

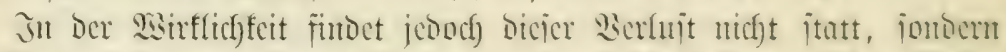

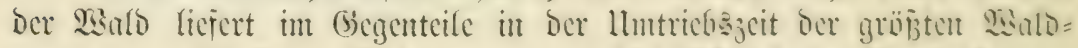

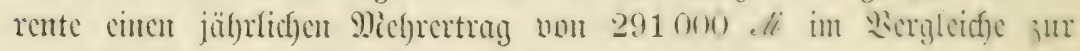
Umtricbszeit Der größ̈ten Bodentente.

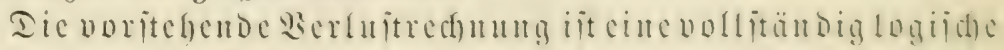
Sonjequenz ber bodenteincrtragalebre.

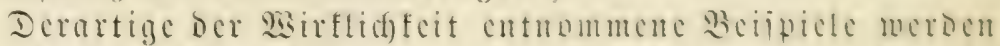

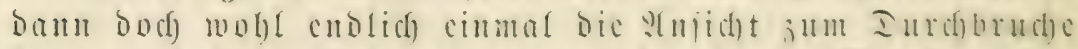

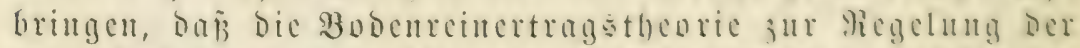

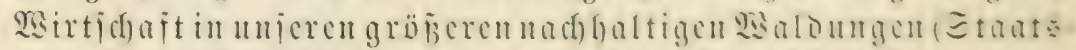

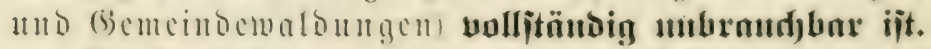

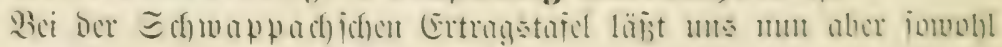

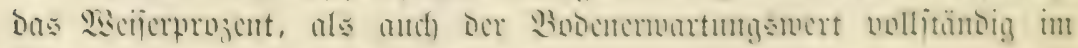
Sticf).

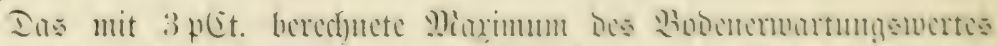

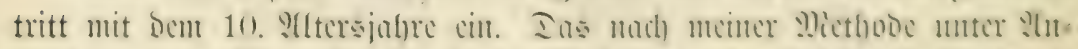

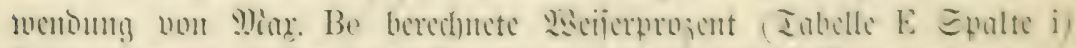

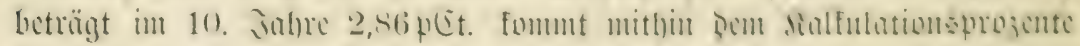
żicutlidf) nat)e.

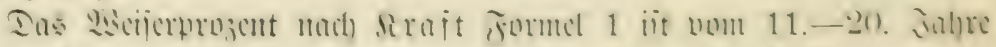
$=1,4 p$ C. uno fällt bis jur Perivos $131-140$ anj 0,3 et. 


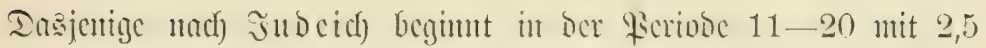
und fälllt bis zur ßeriode 131-140 auj 0,7 pet.

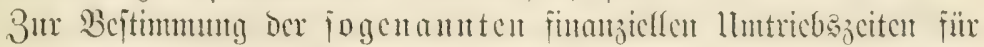

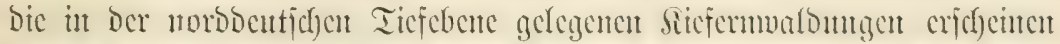

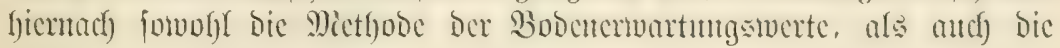
Metlyode Der Wcijerprozente vollitändig unbraudjbar.

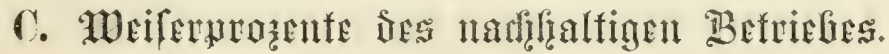

$\S 14$.

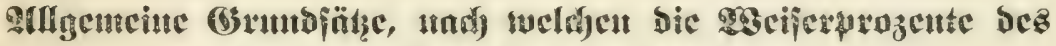

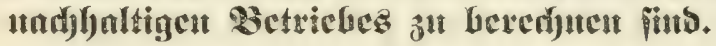

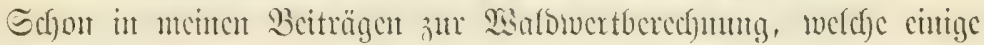

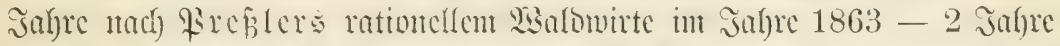

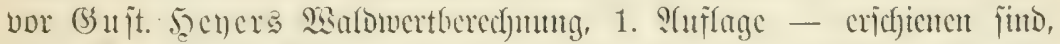

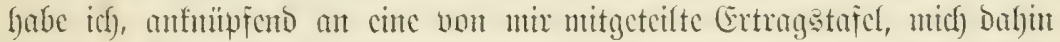

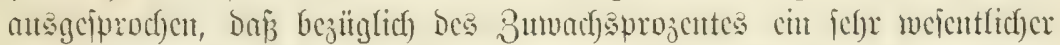

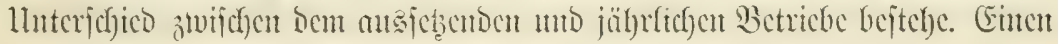

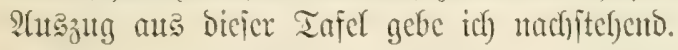

Zusjug aus ber Tabelle E, Ertragstafel über cinch 2rorgen Buḑen= Eod̨wald der III. Bodenflaffe nad Dr. KarI (5rebe in den Betträgen jur Waldwertberedtrung von Bofe, S. 14 2c.

\begin{tabular}{|c|c|c|c|}
\hline \multirow{5}{*}{$\begin{array}{l}\text { Solj: } \\
\text { alter }\end{array}$} & \multicolumn{3}{|c|}{ 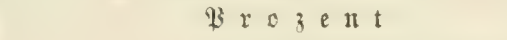 } \\
\hline & \multirow{4}{*}{ 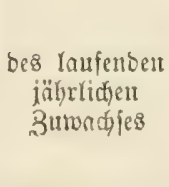 } & \multicolumn{2}{|c|}{ Des gladjbaltsertrnges } \\
\hline & & ofine & mit \\
\hline & & \multicolumn{2}{|c|}{ Dorertrïge } \\
\hline & & \multicolumn{2}{|c|}{ Matzunģorozente } \\
\hline a & $\mathrm{k}$ & 1 & $\mathrm{~m}$ \\
\hline 10 & 26,2 & 37,9 & 37,9 \\
\hline 20 & 11,7 & 17,1 & 17,1 \\
\hline 30 & 10,7 & 12,9 & 142 \\
\hline 40 & 5,4 & 9,1 & 10,2 \\
\hline 50 & 3,6 & 6,5 & 7,5 \\
\hline 60 & 2,6 & 5,1 & 6,0 \\
\hline 70 & 1,7 & 4,0 & 4,9 \\
\hline 80 & 1,4 & 3,3 & 4,1 \\
\hline 90 & 1,0 & 2,7 & 3,5 \\
\hline 100 & 0,8 & 2,3 & 3,0 \\
\hline 110 & 0,6 & 2,0 & 2,7 \\
\hline 120 & 0,4 & 1,8 & 2,3 \\
\hline
\end{tabular}




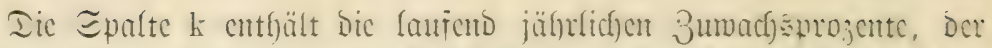

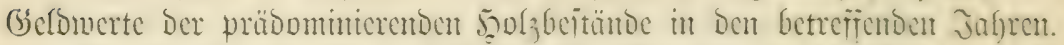

Iic Epalten 1 und $m$ enthalten bie Givojente, roclefe Die ?lomal. norräte nachloltiger Betrichflailen, bei ben llmtricbisciten in Evalte a

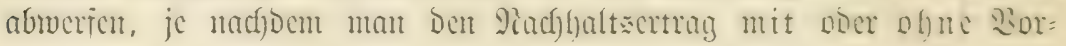
nutzungen - berechunet.

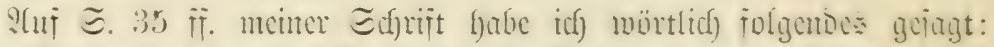

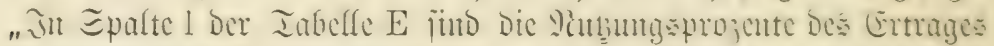

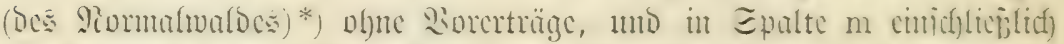

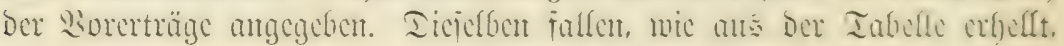

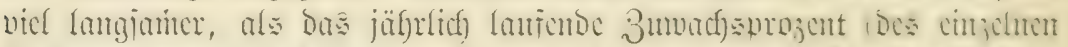

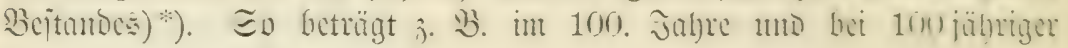
ltmtricbezcit bee Permalivaldes:

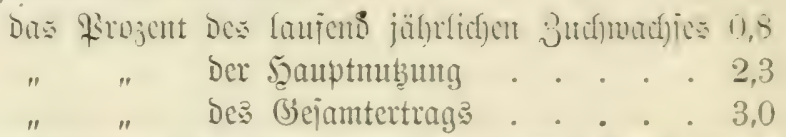

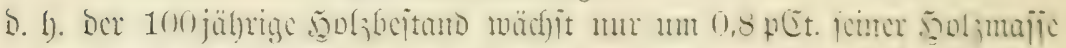

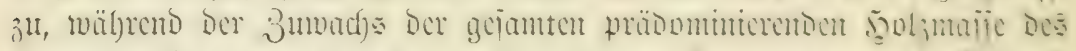

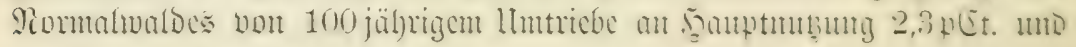
an Gicjantertrag $3 p$ ert. Geträgt.

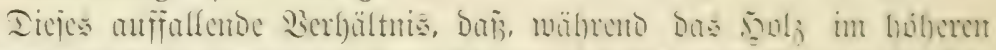

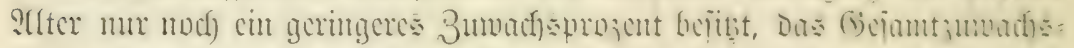

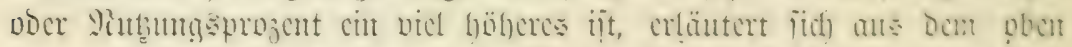

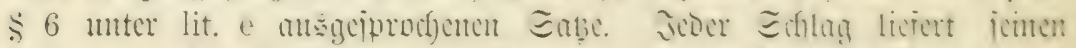

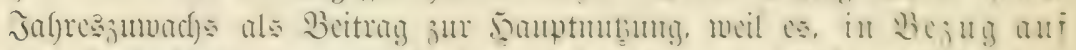

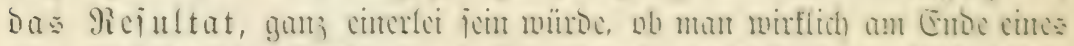

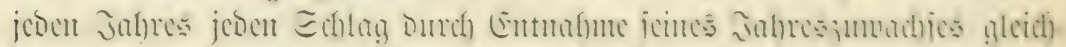

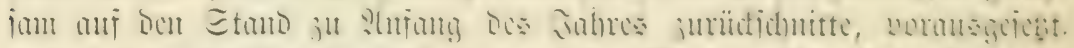

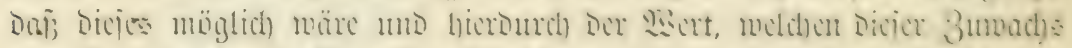

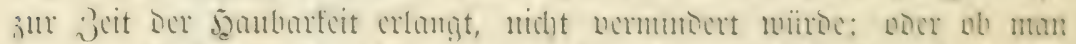

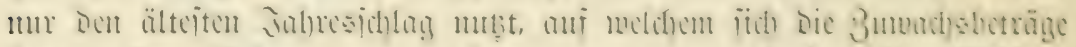

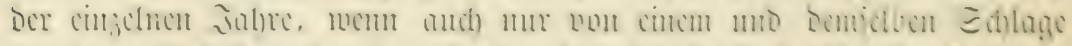

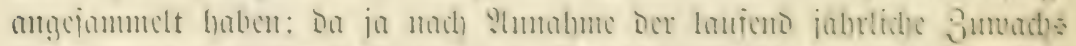

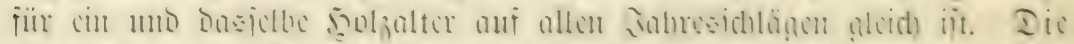

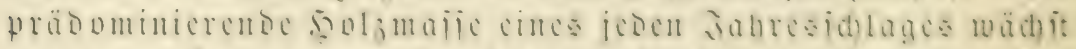

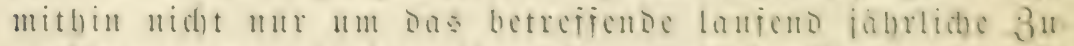

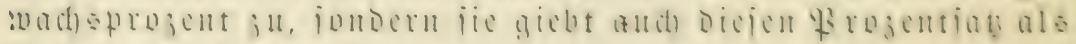

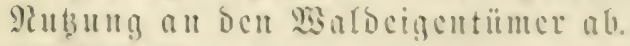

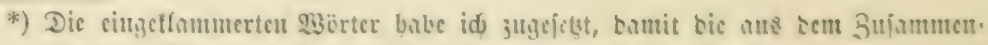

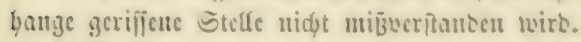




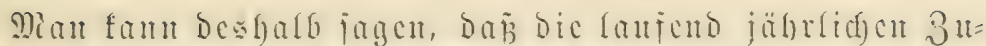

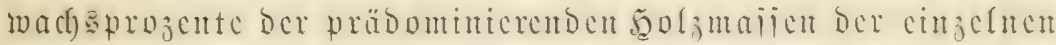

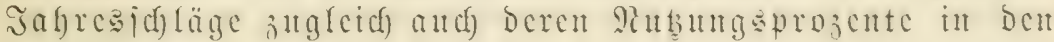
betreffenden Jahren jind."

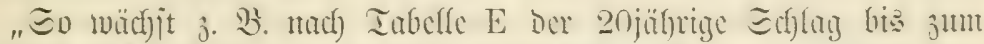

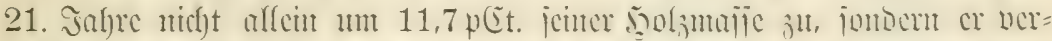

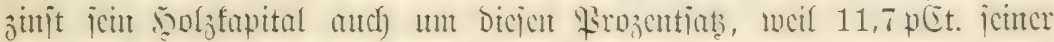

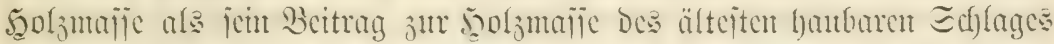

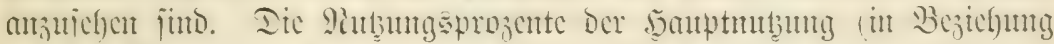

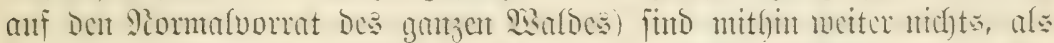

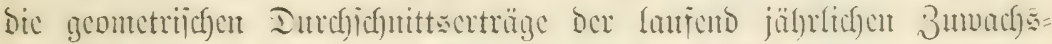

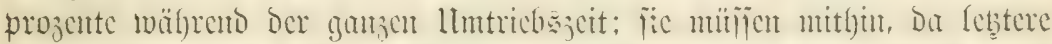

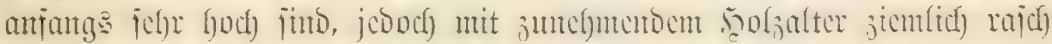
abnefymen, inmer bebcutend höber alg diejelben jein."

Dam babe ich weiter auf Seite 107 \$ 14 gejagt:

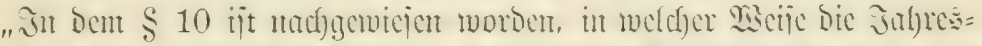

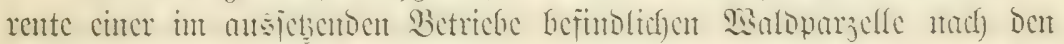

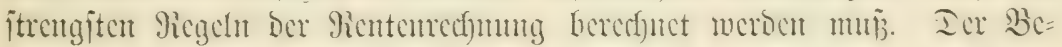

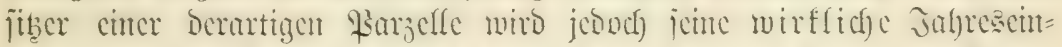

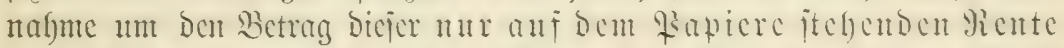
nidjt crböhen, jonbern ar wirb im (jegenteile, Dit nod) anj lange Jufjec

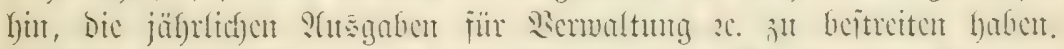
Iic beredyete Rente ijt Deshatb jür icinc siajic, wie idf id on

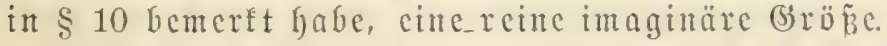

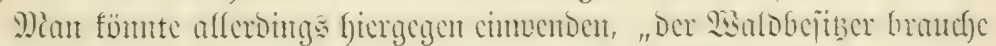

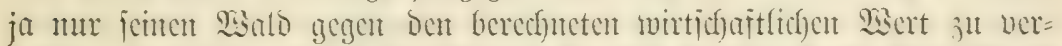

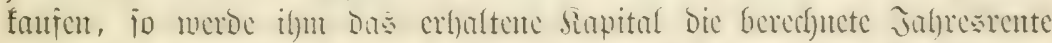

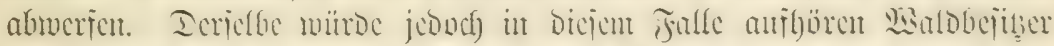

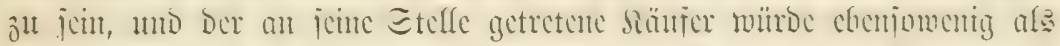

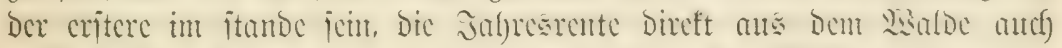

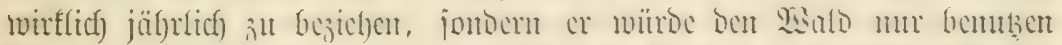

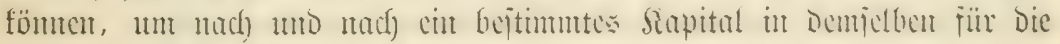
Bufınit anzufammelı.

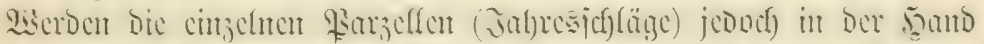

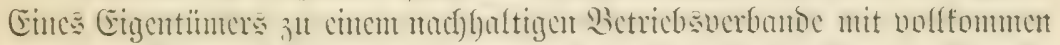

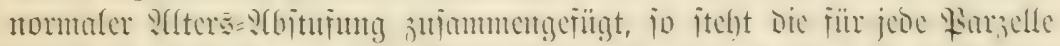

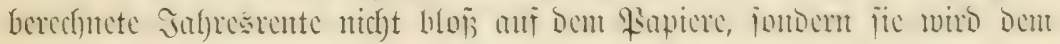

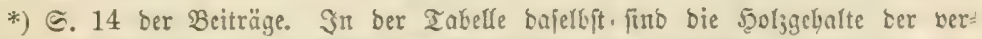

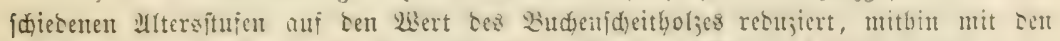
Wertabeträgen in 2trjatz getrad)t, melde fie im falle ses atbriebes bafen mürsen. 


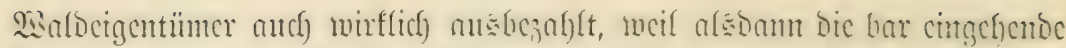

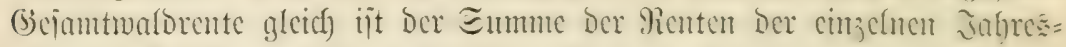

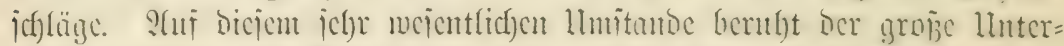

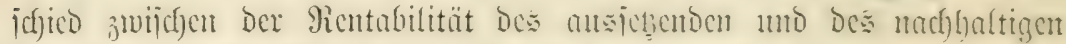
Betriebes, wie wir ipäter jeben werden."

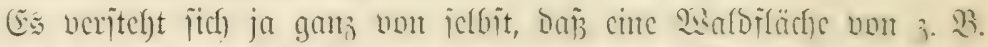

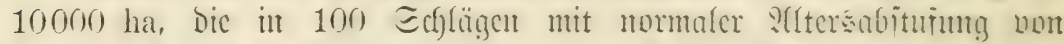

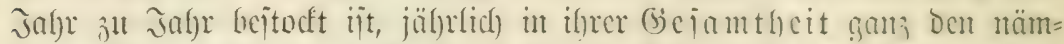

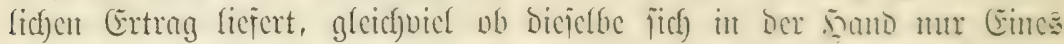
Eigentümers befindet, Docr ob jeder Edylag cinem bejomberen Eigentümer

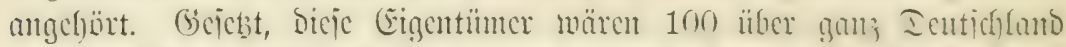

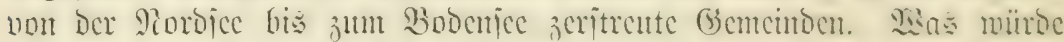

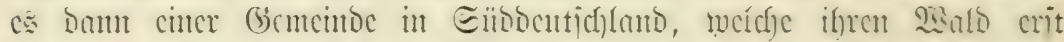

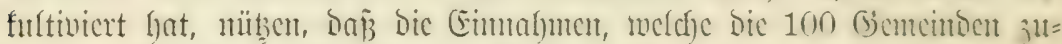

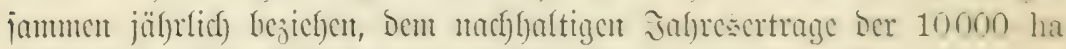

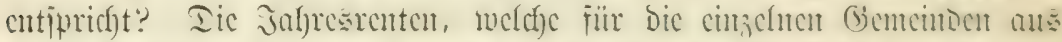

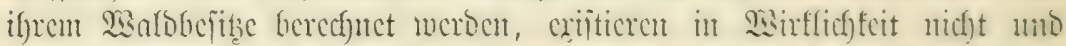

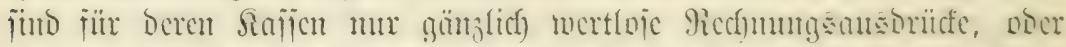

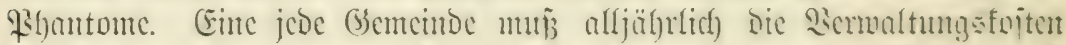

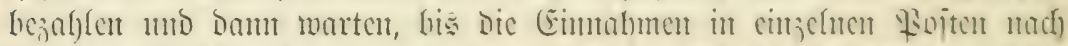

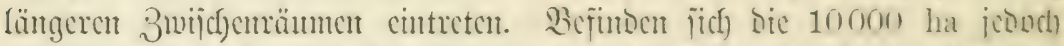

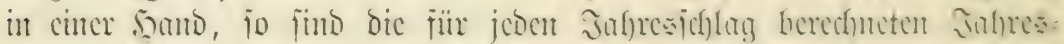

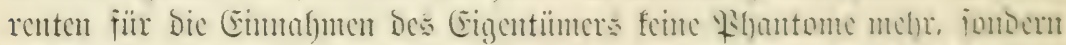

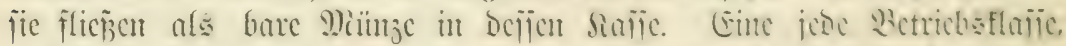

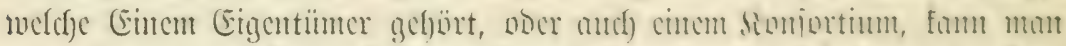

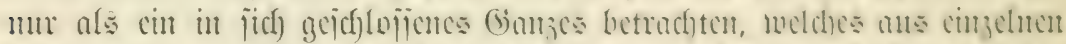

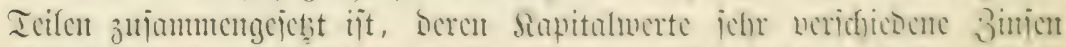

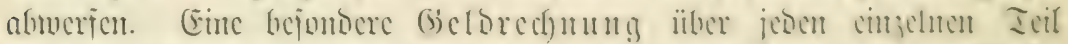

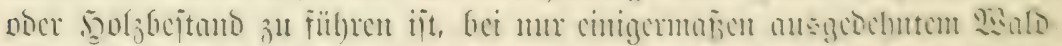

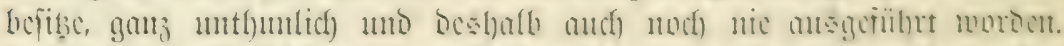

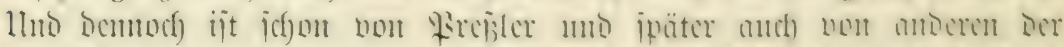

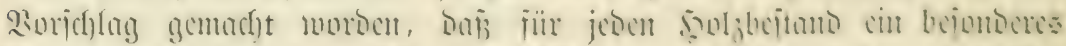

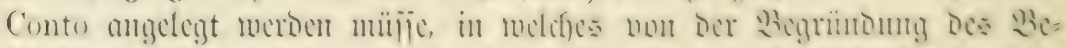

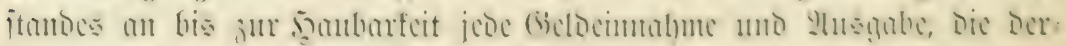

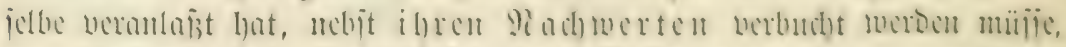

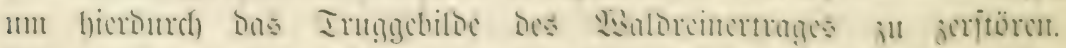

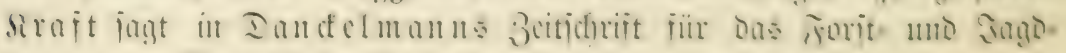

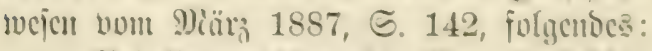

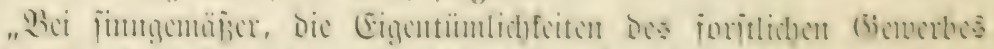

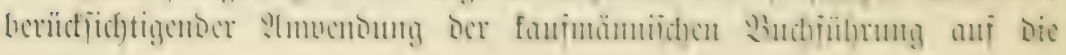




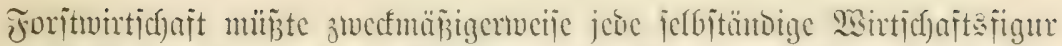
(Iijtrift uno Jagen, bęw. Sfftcilung) ifjr bejonderes Conto bahen mo

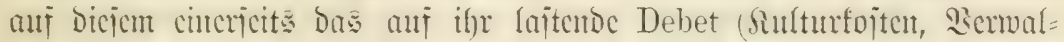

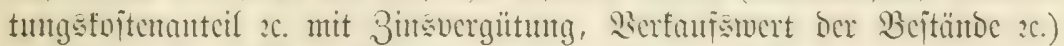

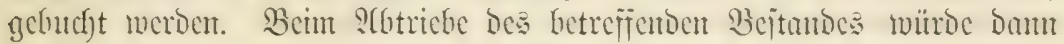

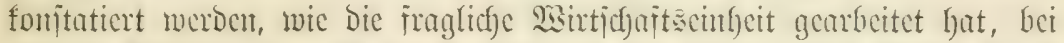

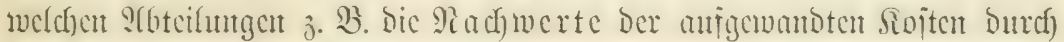

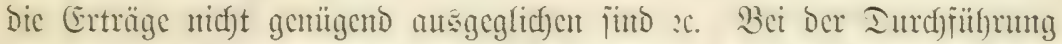

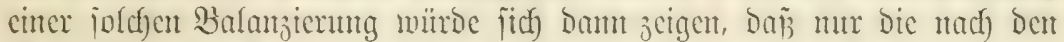

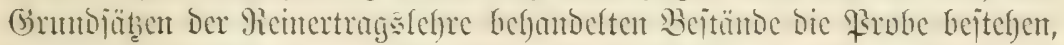
mogegen alfe übrigen cine Berlujtwirtichait ofjenbaren mïtoen."

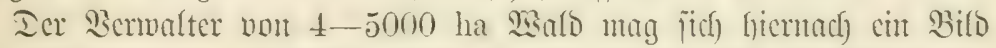

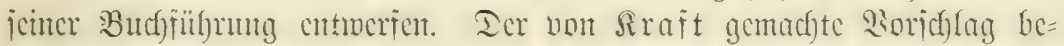

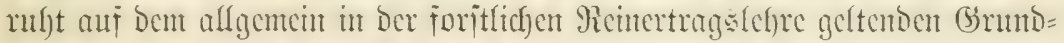

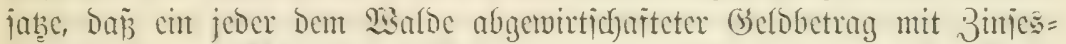

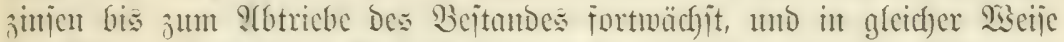

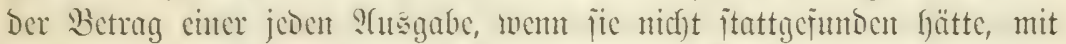

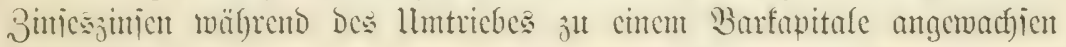
jein twiurbe.

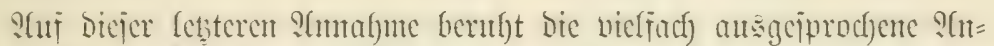

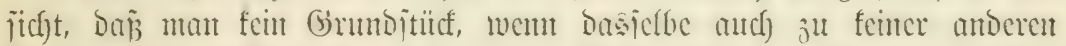

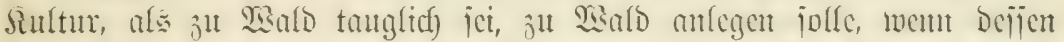

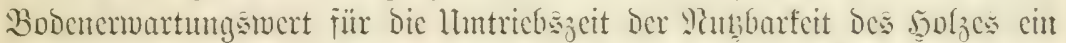
negativer jec, weil ja bic für bic Sisalofultur nötigen fiojten in bicjer

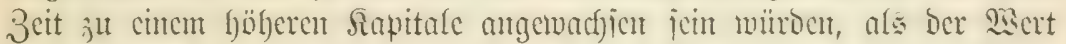

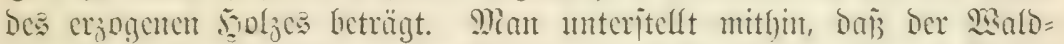
cigentümer bet lluterlajjung ber fultur jebenfalls bie betreffendon fojten

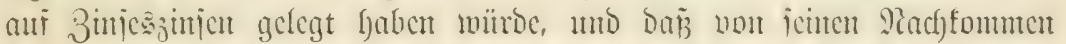

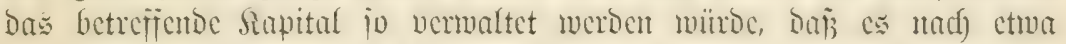

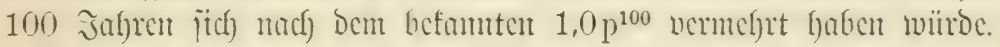

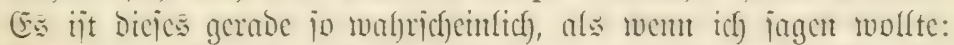

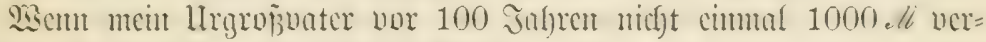
loren babe, jo würbe idf) jebt cin Bermögen von

bej̈isen.

$$
1000 \times 1,03^{100}=1000 \times 19,223=19218 \text { ॥ }
$$

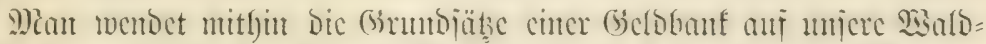

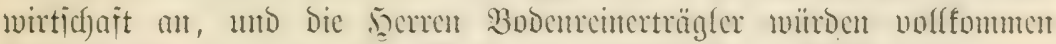

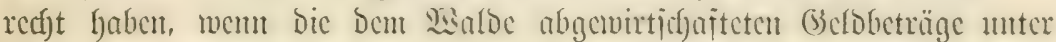

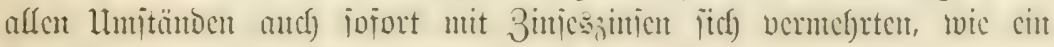
Betrag, Den mant in cine Sparfajic eingelegt hat. 
Die Marbmirticjait erzeugt jeboch) eincut (begenitanto des täg= Yidfen Berbrautches, Dejien Bseld= wert tidd)t氶 iveniger, als auf Bimjeszinjen gelegt twiro. Mlag man burrd bie angebentete $\mathfrak{B}_{\text {utd }}=$ fülyrumg aud nod) jo gropie (beclo= verluite herausredfune, io be= iteben biejelben bodf nur auf bem Papicre uno find mitfju mur \$shantajiegcbiloe. Mian wiro burd) alle bieje Riedjuntugen bie Thatjadje nidfyt wegredfunen fümten, Daỉ bie Şodjwaldungen Der Deutjojen Staaten bei ben

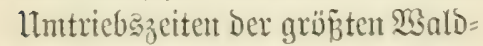

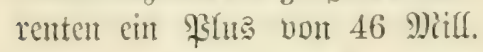
Miant gegeniber Den 11 mttriebs: zeiten Der gröïten Sobemuerte jäfrlich) rein in Die Salijent liefern, seem bicjelben in ber 19 erje be= ftanden find, wie ich) in baurs CentralGlatt vom ?tatguit 1888, S. 445 , Tabelfe D angentonmtent babe. Die Secren S3obentecin= crträgler berecfjuen jebod) bei bic=

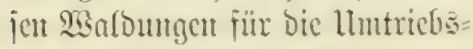
zeiten Der böcbiten פEaldorenten

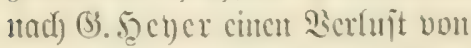
jäf)rficl) 106461298 dt. (M) (Mn vergl. Baurs Eentralblatt vom Pioventber 1886, ङ. 557 แm vou Fefturar 1889, ङ. 91). Jit Don $\$ \$ 4$ u. 13 bicjer E(f)rift loate idf) nod) weitere S3cipiete bicjer antyeb-

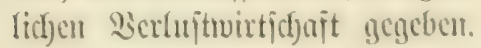

Fïr Dicjenigen, iveldfe nichtt

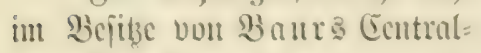
blatt find, jiinge idf) bie genaunten fretben Inlletlen hier an.

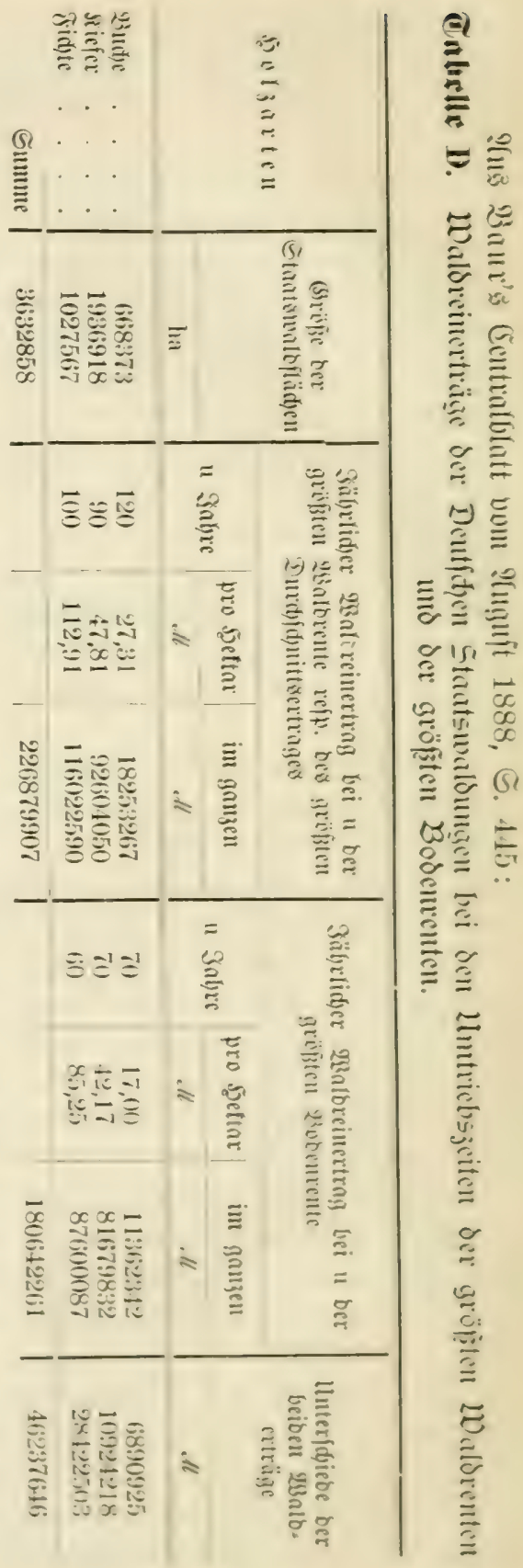




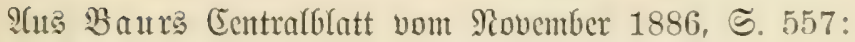

Caluelle C. ïtber den Derlujt, der in den deutihen Staatswaldutgen Jurch Einhaltung der Umtricbsjeiten der größ̨ten Waldrenten gegenïber Jen Untricbsjeiten der gröfiten Bodenrenten entitehen foll, mit Ein= fügung der Bodentwerte.

\begin{tabular}{|c|c|c|c|c|c|c|c|}
\hline \multirow{4}{*}{ 5̧oljarten } & \multirow{3}{*}{$\begin{array}{c}\text { (3) rößze ber } \\
\text { Stantz: } \\
\text { maloflädjen }\end{array}$} & \multicolumn{2}{|c|}{$\begin{array}{c}\text { Umtriebsizeit ber } \\
\text { gröfiten Boben }= \\
\text { rente }\end{array}$} & \multicolumn{2}{|c|}{ 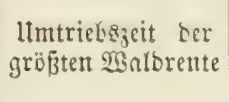 } & \multirow{2}{*}{\multicolumn{2}{|c|}{ 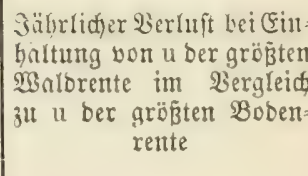 }} \\
\hline & & \multirow{2}{*}{$\begin{array}{c}u \\
\text { Sabre }\end{array}$} & \multirow{2}{*}{$\begin{array}{l}\text { Boben }= \\
\text { erwartungs } \\
\text { werte } \\
\text { pro Seftar }\end{array}$} & \multirow{2}{*}{$\begin{array}{c}\text { ul } \\
\text { Sabre }\end{array}$} & \multirow{2}{*}{$\begin{array}{l}\text { Boben = } \\
\text { erwartungs }= \\
\text { werte } \\
\text { pro Şeftar }\end{array}$} & & \\
\hline & & & & & & pro jeftar & im ganzen \\
\hline & ha & \multicolumn{2}{|r|}{ ell } & \multicolumn{2}{|r|}{$d l$} & \multicolumn{2}{|r|}{$d l$} \\
\hline $\begin{array}{l}\text { Budde. } \\
\text { fitefer. } \\
\text { fidute. }\end{array}$ & $\begin{array}{r}668373 \\
1936918 \\
1027567\end{array}$ & $\begin{array}{l}70 \\
70 \\
60\end{array}$ & $\begin{array}{r}85,36 \\
362,56 \\
986,59\end{array}$ & $\begin{array}{r}120 \\
90 \\
100\end{array}$ & $\begin{array}{r}16,61 \\
267,94 \\
631,48\end{array}$ & $\begin{array}{l}19,30 \\
13,98 \\
64,70\end{array}$ & $\begin{array}{l}12899599 \\
27078114 \\
66483585\end{array}$ \\
\hline Summe & 3632858 & & & & & & 106461298 \\
\hline
\end{tabular}

Die Bobenerwartungswerte fub rein exfl. V angegeben.

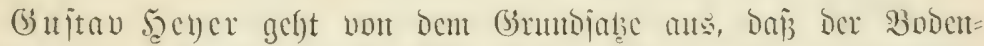

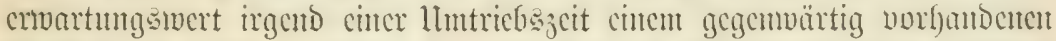

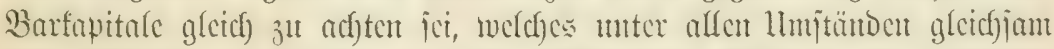

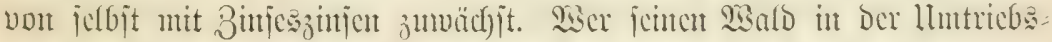

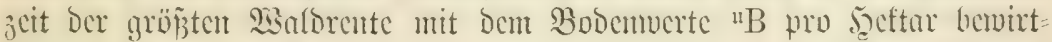

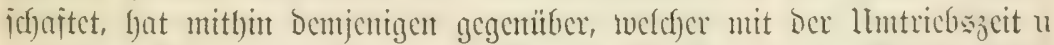

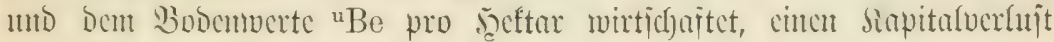
von $\left({ }^{\mathrm{u}} \mathrm{Be}-{ }^{\mathrm{u}} \mathrm{B}\right)$ pro Ђeftar.

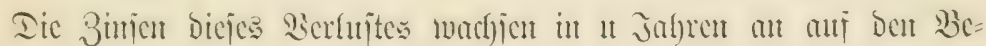
trag von $\left({ }^{u} \mathrm{Be}-{ }^{u} \mathrm{~B}\right)\left(1,0 \mathrm{p}^{\mathfrak{u}}--1\right)$.

Der Serluit pro Salyr umo Seftar beträgt mitlin

$$
\left({ }^{\mathrm{u}} \mathrm{Be}-{ }^{u} \mathrm{~B}\right)\left(1,0 \mathrm{p}^{\mathfrak{u}}-1\right)
$$

11.

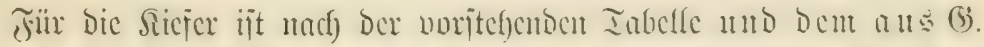

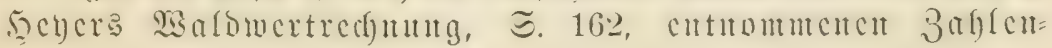
beipiele $\quad$ uBe $=362,56 \mathscr{M}$

$$
\begin{aligned}
& \text { u } \mathrm{B}=267,94 \\
& \mathrm{u}=90 \\
& \left(1,03^{90}-1\right)=13,3,
\end{aligned}
$$




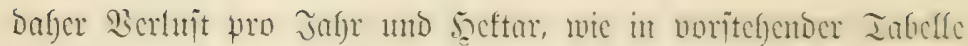

$$
\frac{(362,56-267,94)(13,3)}{90}=13,98,
$$

Sierluit Der Siejer $=1936918 \times 13,98=27078114$.

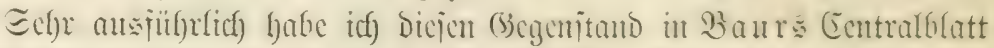

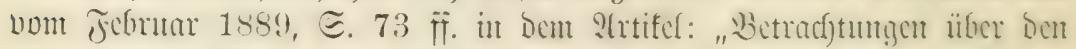

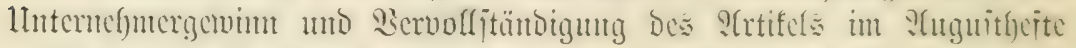

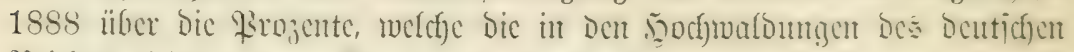
Mecidfes nicocrgelegten Sapitalien abmerien," befoundelt.

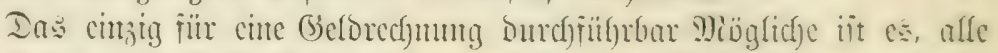

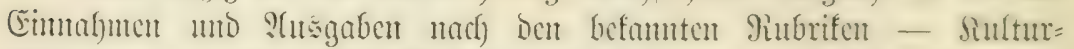

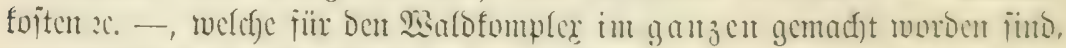

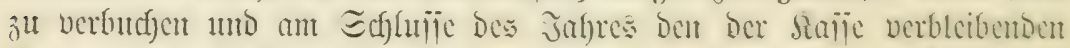

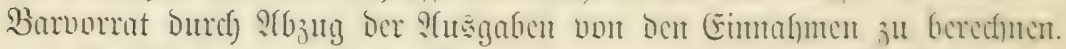

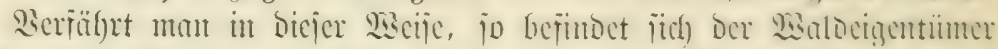

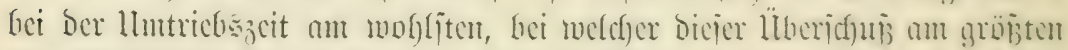

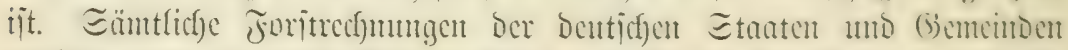

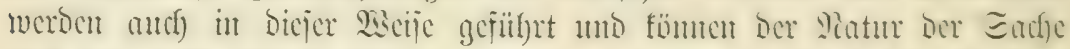

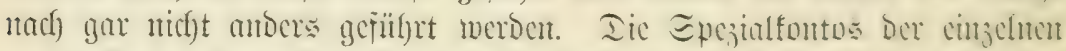

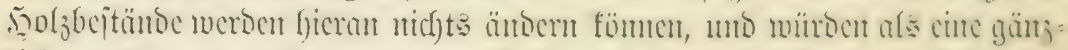

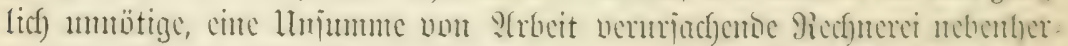

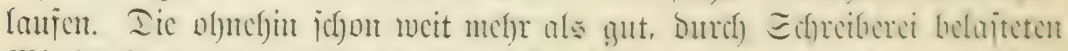

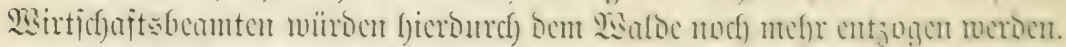

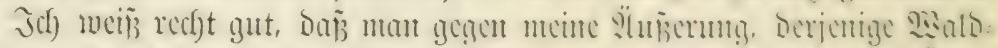

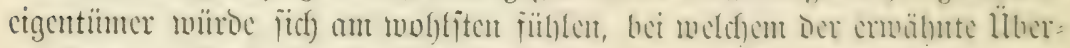

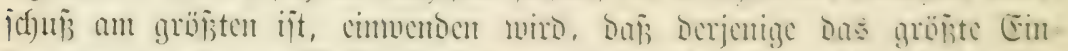

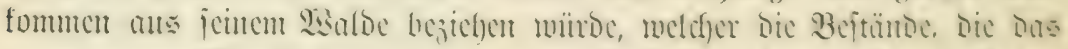

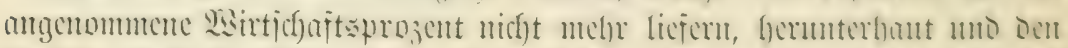

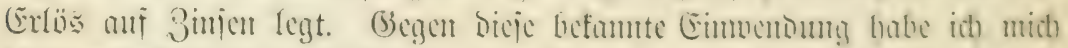

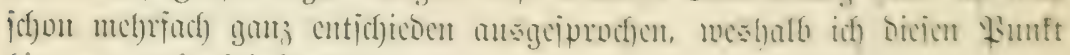
fier mux nebentbei berïl)re.

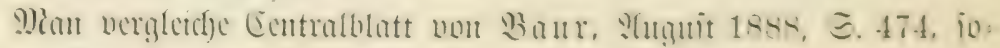

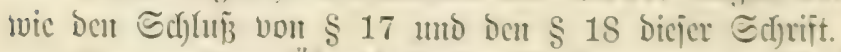

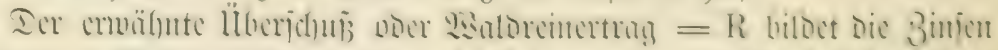

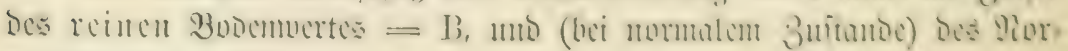
mafuorrats $=\mathrm{NV}$.

(Es ift buffer imuner $(B+N T) 0,0 p=R$.

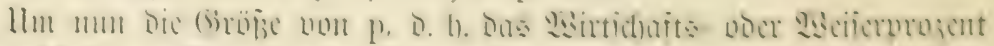

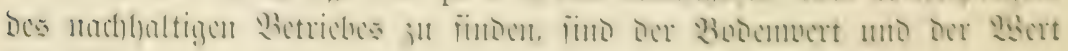

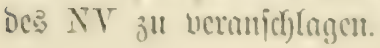




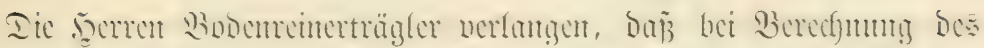

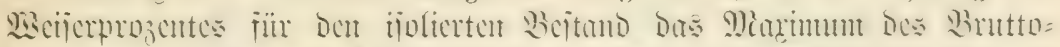

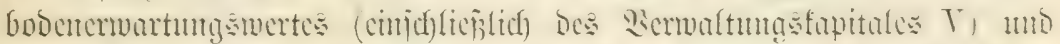

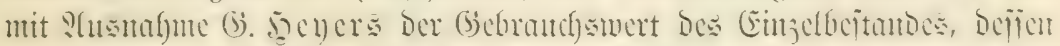

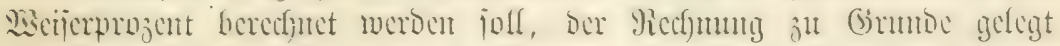

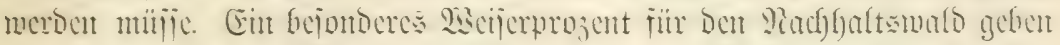

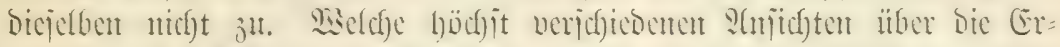

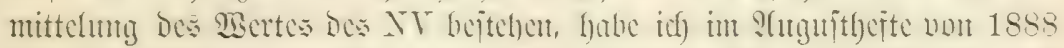

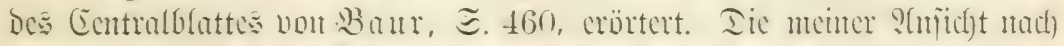

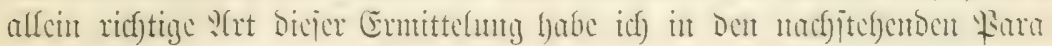
graphen bejchrieber.

\section{$\$ 15$.}

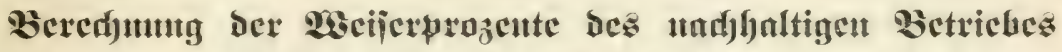

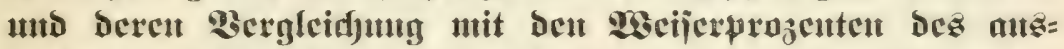

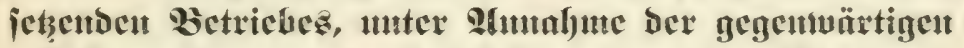

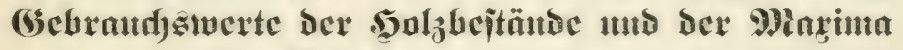 Der Sobencruatuntgsiucte.}

(Siche die Iabellen F, G und H auj S. 48, 49 mo 50.)

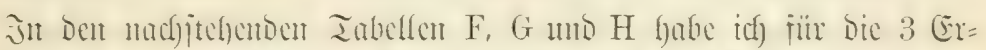

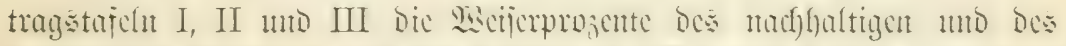

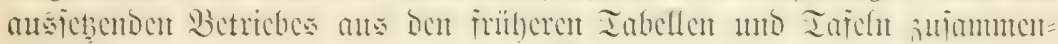

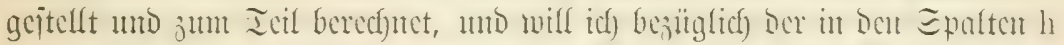

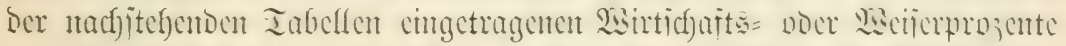

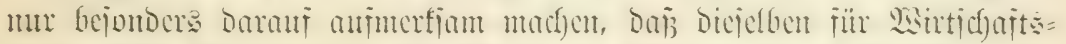

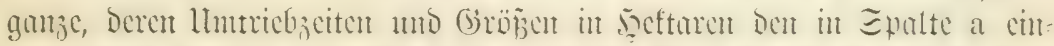

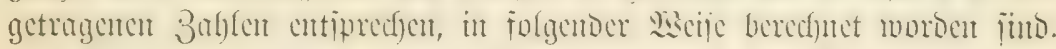

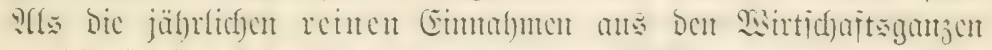

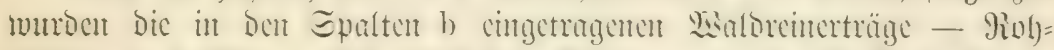

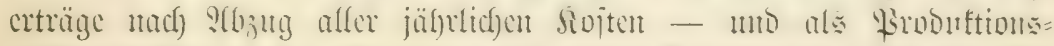

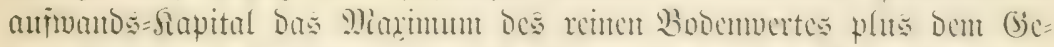

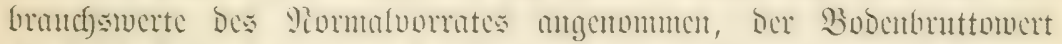

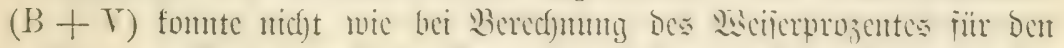

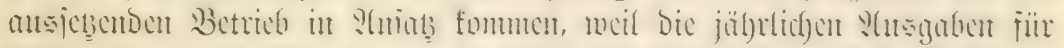

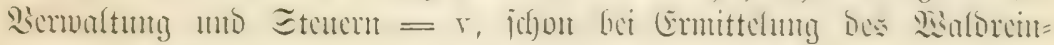

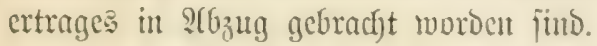

In ben Epalten $\mathrm{i}, \mathrm{k}, 1$ mo m ber Tabelfen twurden bie 23eijer=

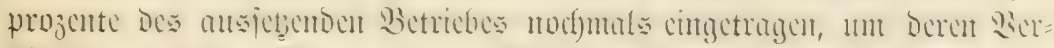

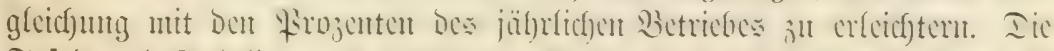

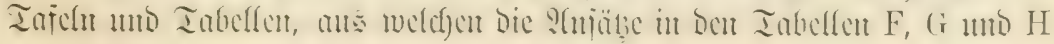




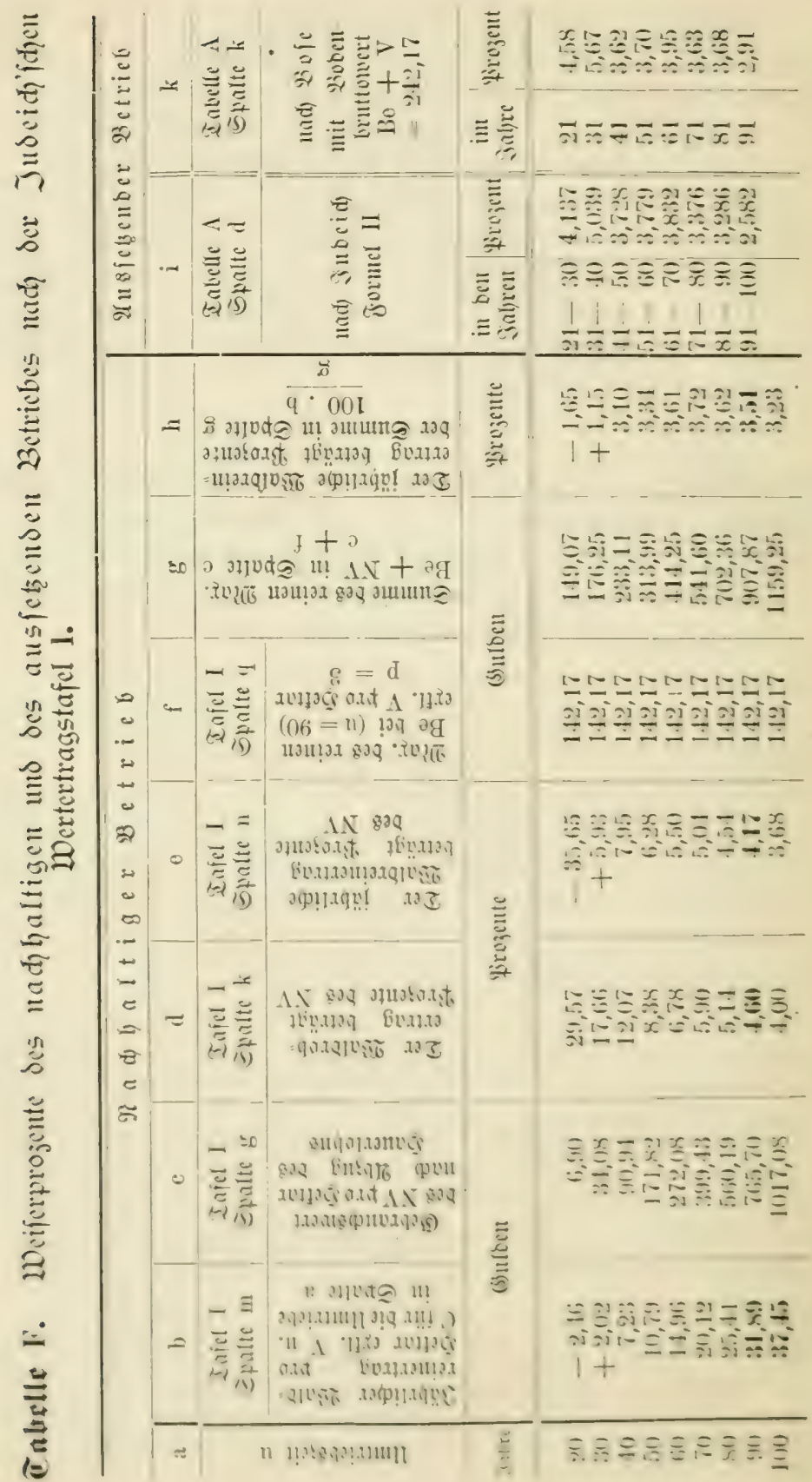


C. 23ciferprejente Ecs nndibaltigen Betrictes. § 15 .

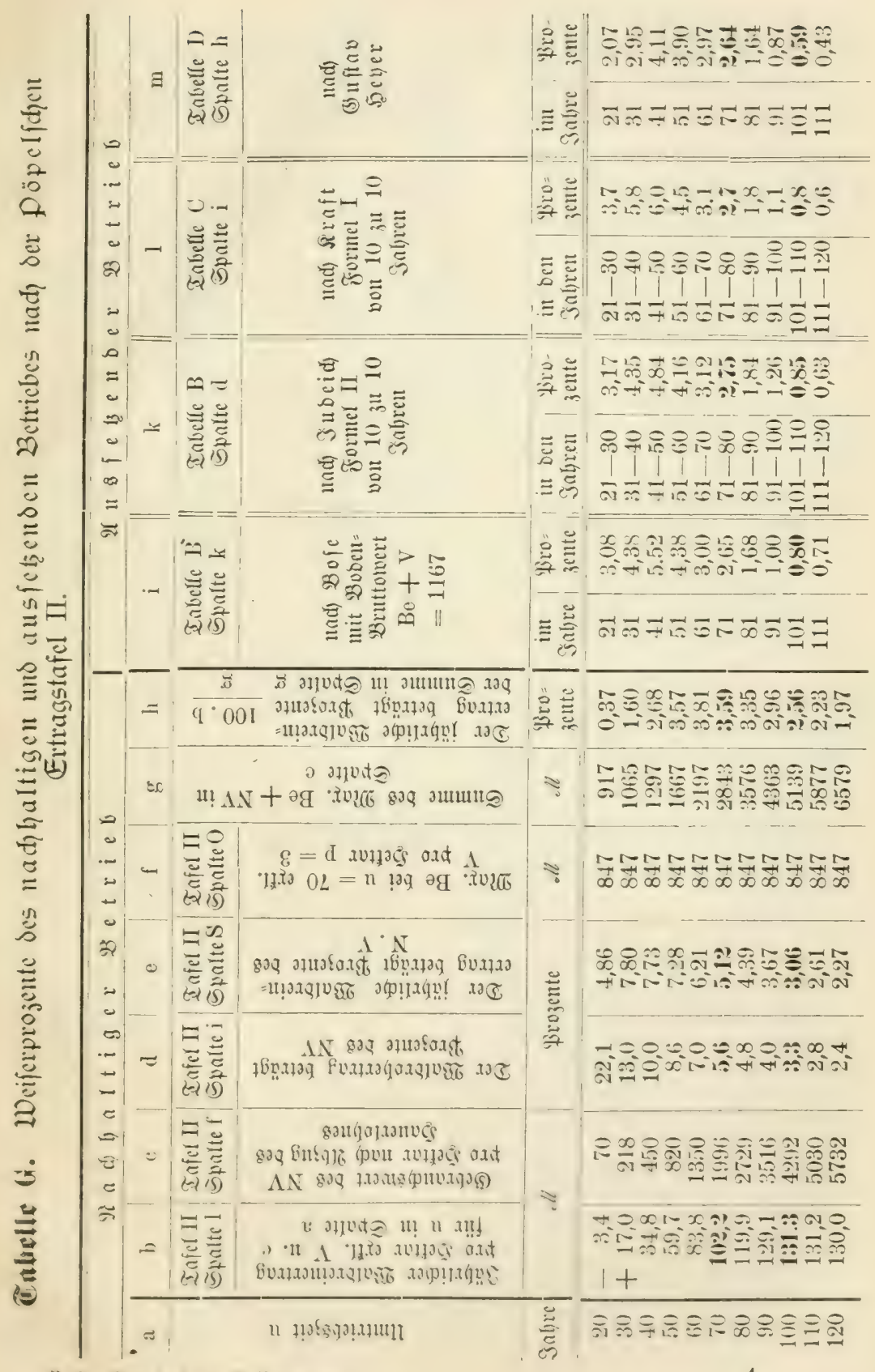

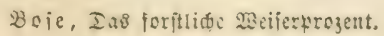




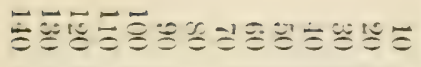

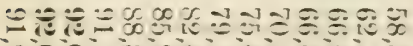

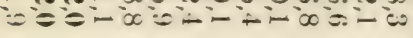

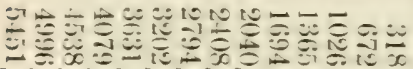

or

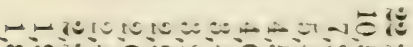

oreiston

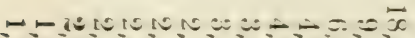

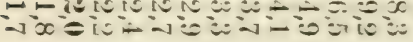

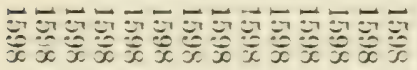

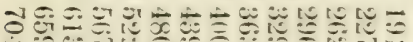

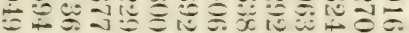

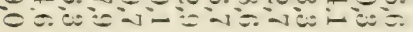

br-ch-

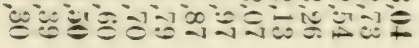

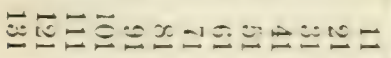

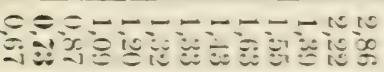

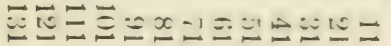

$=0=0=0.0=00$

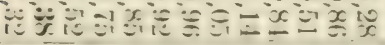

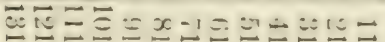
11111111111

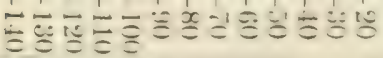
cecen

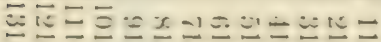
1 1

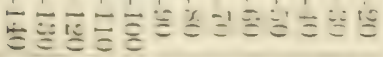
ces iv

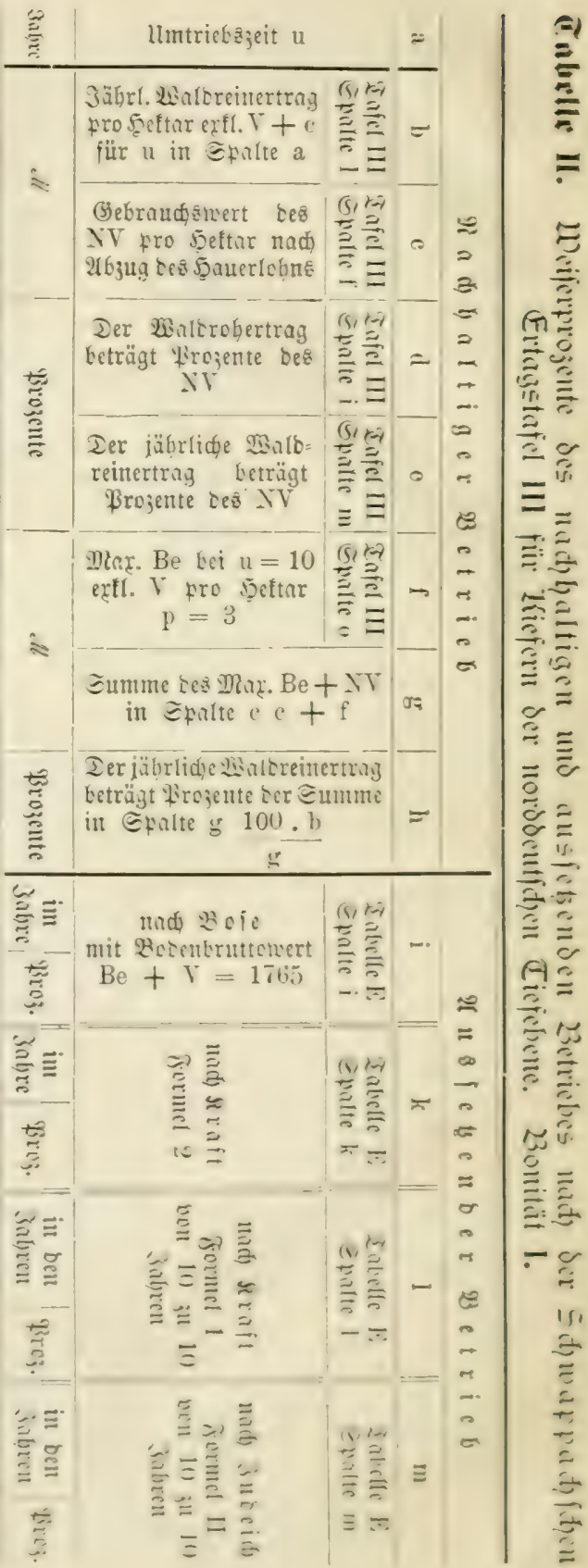




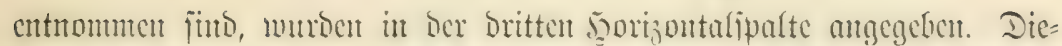

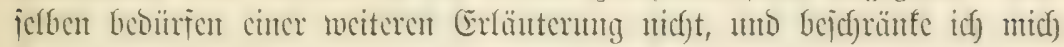
beshalb mur auf Servorbebung einiger $\mathfrak{B}$ untte.

Für Dic Jubeichid) Ertragstäed letragen hei ber llmtricbs=

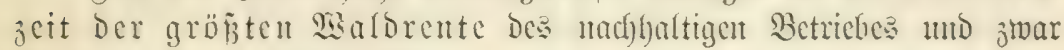
bei $u=100$. Inbelle $\mathrm{F}$

Der Maldrohertrag \$rozente Des NV . . . . . 4,00

Der Maloreinertrag \$rozente bes NV . . . . . 3,68

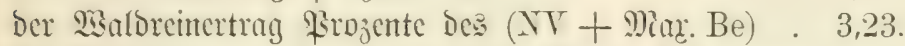

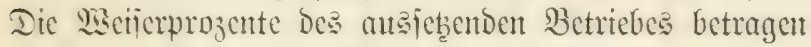

fïr Den 91 jährigen Bejtand nad B bje . . 2,91

fïi dic Periode von 91-100 nad) Jubeidy . 2,58.

Fitr bie \$öpelide Ertragztajel betragen bei ber llmtricbe

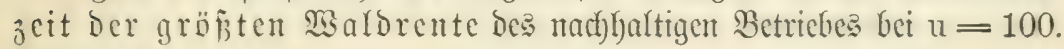
Tabelle $G$

der Maldorohertrag Prozente dę NV . . . . . 3,30

Der Maldoreinertrag \$rozente Des NV . . . . . 3,06

Der 2 aafdreinertrag Prozente bes (NV + Mar. Be) . 2,56.

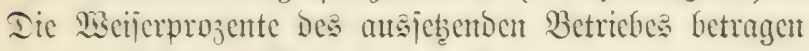

für Den 101 jährigen Bejtand nach) Boje . . . . . . 0,80

für Die \$Beriode von 101-110 nad) Iudeid) . . . . . 0,85

" " " " " Rraft (Form. 1) . . 0,80

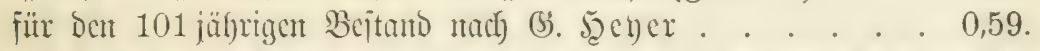

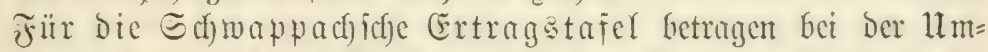
triebszeit Der größten Maldente Des nadjhaltigen Betricbes bei $u=120$. Iabelle $\cdot H$

Der Walbrohertrag \$rozente Des NV . . . . 2,2

ber $\mathfrak{W a l b r e i n e r t r a g ~ \$ r o z e n t e ~ b e s ~ N V ~ . ~ . ~ . ~ . ~ 2 , ~ 2 , 0 ~}$

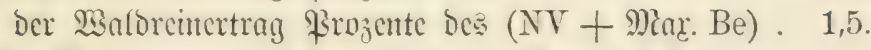

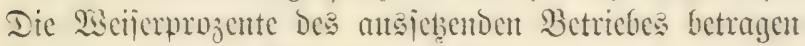
für ben 121 jährigen Bejtan nadj Błoje . . . . . 0,73 " " " " תraft (Form. 2) . . 0,38 fïr bic Pseriode bont $(121-130)$ nad) Siraft (Jom. 1) . . 0,40

" " " " " "ubeid) (Form. 2) • 0,80,

(E⿱

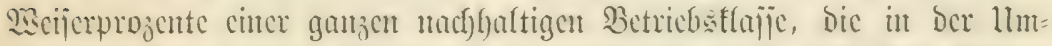

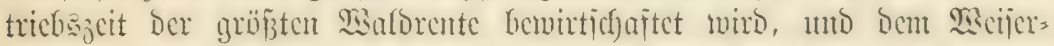

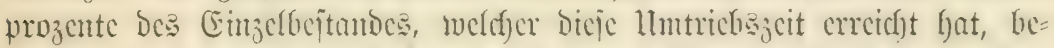

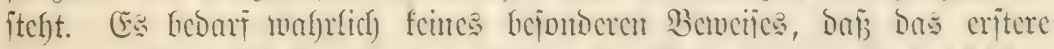

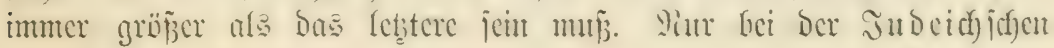


Ertragstajel tritt biejer Inter= jđjied nicht jo jtarf heroor. F mag Diejes Dafler fommen, Dá̉ Dieje Iafel nidjt Der Minflict)= feit entnommen, fonbern alફ Refrbeipiel fonjtrutert worden zu idjeint.

$\S 16$.

23cijerprozente DeE ntud)= faltiget Betricbes utter \&utucubuth Der

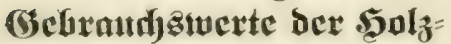
Gejtünto uto veridjebener Bobenwerte.

Bei ben im vorigen $\mathfrak{B a r a}=$ grapl) ausgefülyrten Rechnungen wurden die Miarima Der Boben= crtwartungatwerte und bie (Bes= braud) stwerte Der Materialvor= räte zu Grunto gelegt; - näm= (idd) Diejenigen Merte, für toelche jeber einzelute Fejtmeter würode verfanti meroen fömen, twent

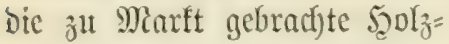
majje ben jeityerigen Eimjchlag, bei tweldyem fich) Der Taujdjtwert gebilloct lyat, nicht überiteigt.

Dẩ bei ben von umjeren

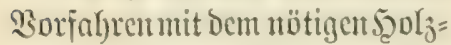
vorrate crerbten 13 aldoungen bie ?tmalyme:

"Dic Miecfjum niilije jo

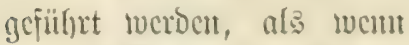
wix Dic MRarima ber Boden= cruartumgasuerte gleidjam bor it Dem Buben nicber: gelegt lyätten,"

ciuc Durdfaus veriefles mo

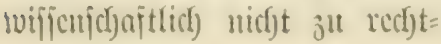

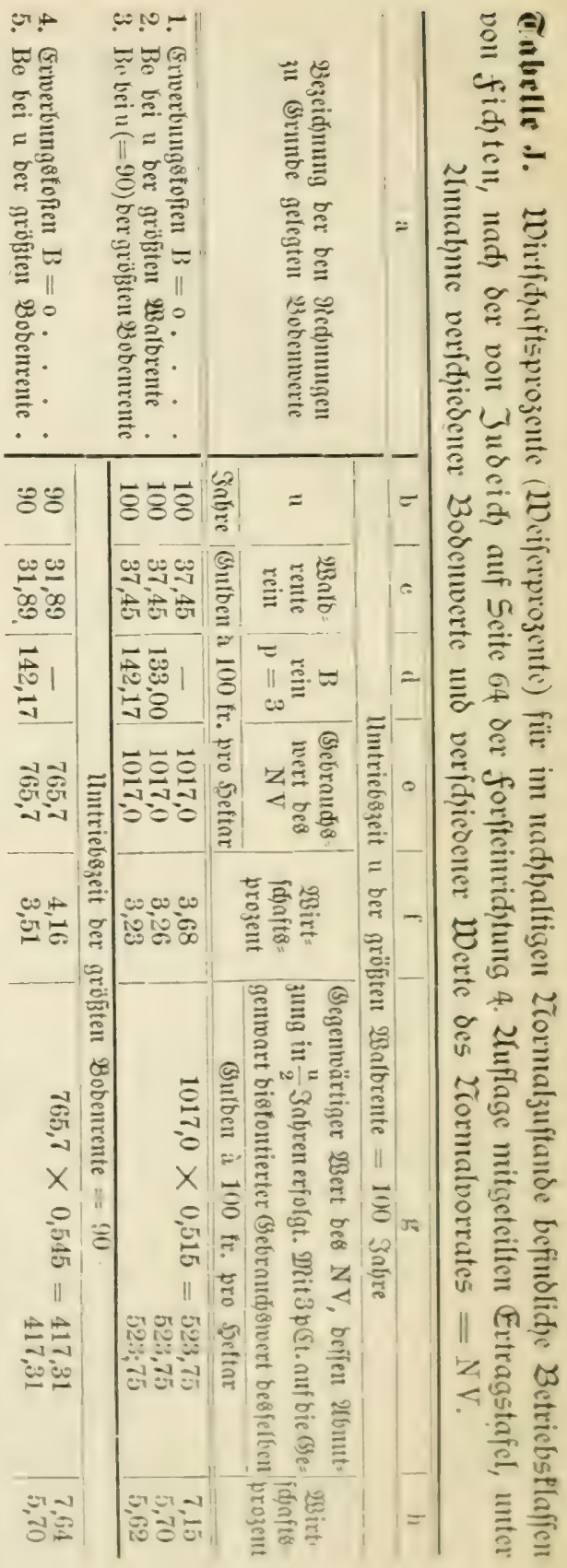


(1)

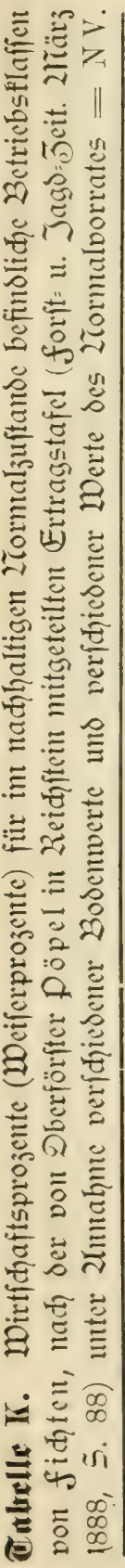

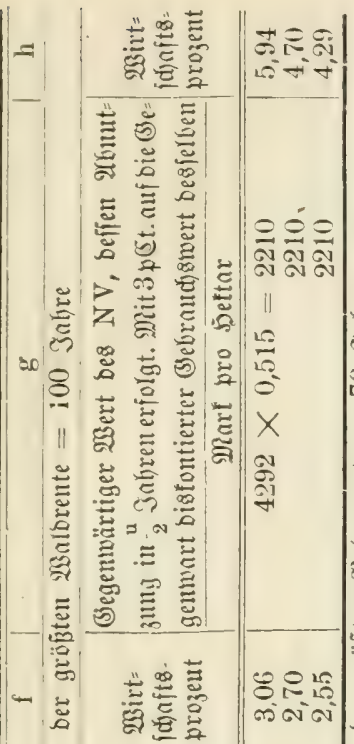

\section{-} |

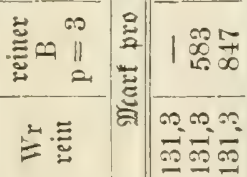
$=$ (5)

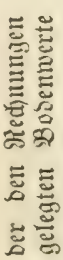

焉弟 จi⿻i力i

fertigenoc jei, foube idf) auf S. 451 de? ?(utguttflectes bou 1888 แ11 สแก $\approx .84$ des Fefruarlyeftes

N几N

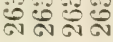

3

X

ติ

I

Nos In

:

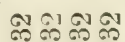
का von 1859 Dce $\mathfrak{B}$ a ll: id)en Eentralblattes ausfiil)rlich machge= weicjen 1ttro oben im $\$ 6$ aud fuly be= grititioet.

Dic Erverbung $=$ foiten Des Siodert jino bei bicjen $\mathfrak{W a l}=$ Duntgen gleidf) Rull. Mill man jedoct) ci= nen Bodcmucrt in 2ujab britgen, jo foum mant mit bem= jelben Picclite, wie Das Miarimum dea Budencruartung

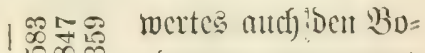
Dencrwartungatwert

-0.0.8. Der IIntrichajut Der 今ิ antuetumen.

오ㅇㅗㅗ (Siche dic Tab.J, Ki un L anf $\subseteq .52$, 53 utio 54.)

S̈l Den Tublilen J. K แmb L galle idf - 를 - $\xi_{3}$. in Don Epaltul a $\|=5 \frac{5}{2}$

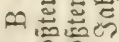
류류: bia f Dic Mcifcr= prozente doce nach = क. E $=\approx$ fïr cincu joben bie=

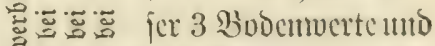

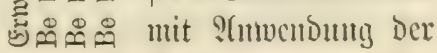

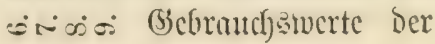




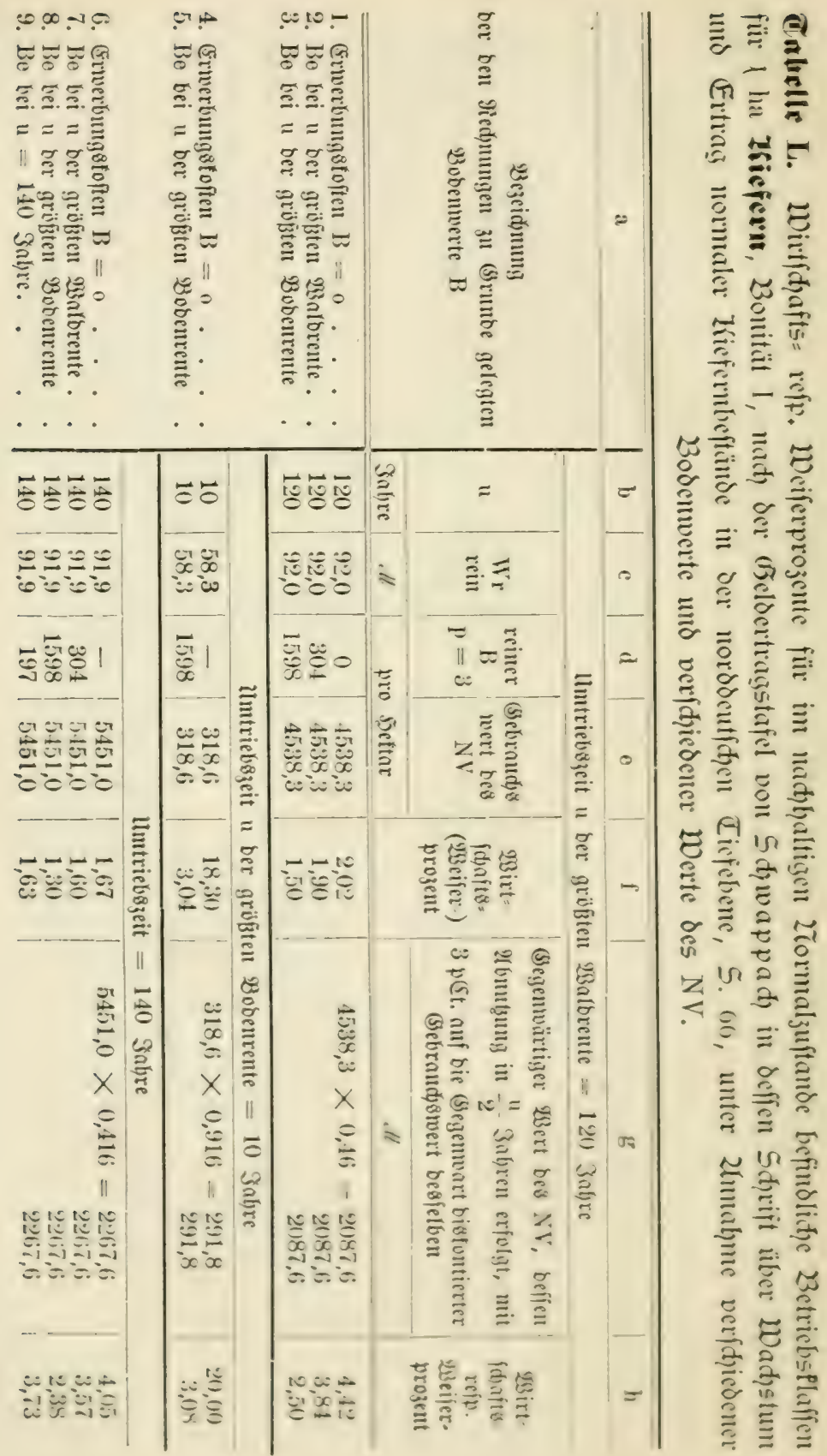




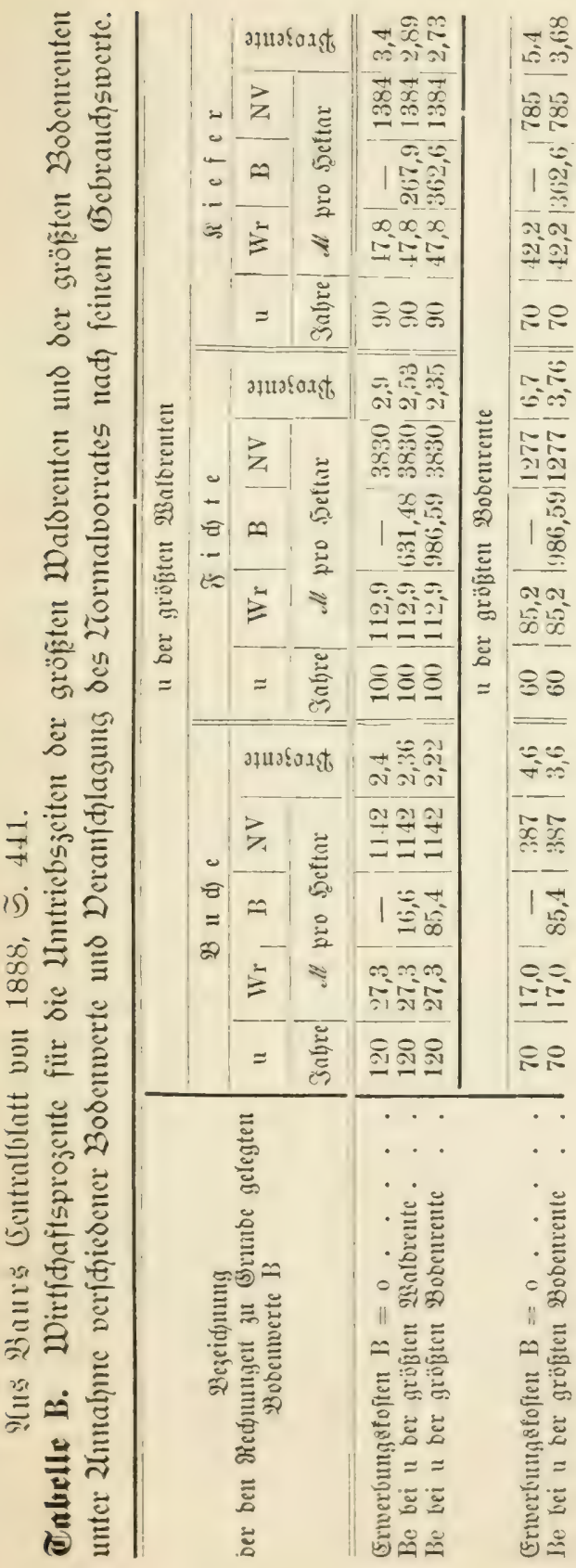

Rormalvorräte fïr verichic= bene Ilmtricbisciten berech)= uct. Iic in Epaltc f cint= getragentu 2 sirtichajt: voer 2iscijerprojente betragen, je nadjobum mant ben cincu poer anteren ber genamten $\mathrm{B} \mathrm{D}=$ Denuerte in Stujabs bringt, für bic llmtrichaseiten ber grö $\tilde{B}=$ ten 2ialdorenten:

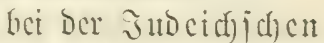
Inid $3,23-3,68$ pet., bei oce töpelide at Iajel 2,55-3,06 "

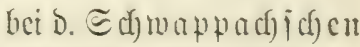
Tafel $1,50-2,02$ ?ack ber von mir it Baur: Ecutrallolatt vom : (uguit 1888, こ. 441 gc= gebenen Tabclfe B, socldye idf ncbenjtelyend bier jolgen

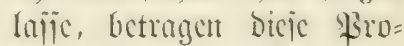

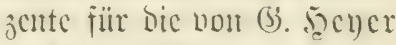
in jemer 2ibalowertrect)ung veröffentlid)ten Ertragä= toujclı für D. BHuclye $2,22-2,40$ pCt., " " Fitcftc 2,35-2,90 " "Sicicr 2,73-3,40 "

(5) gel)t als bicien 6 Ertragstajch sitr (B) crillục

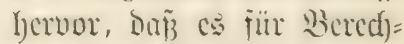

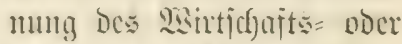
2iscijerprojents bes nad)hal= tigen Betriches feine jehr mejentlichelluteridjico muadjt, weld)en ber brei genomuten 3obcumerte man ammimmt. Iicie llnteridjecos ariffeincu iajt als verichmutocmo, mem 


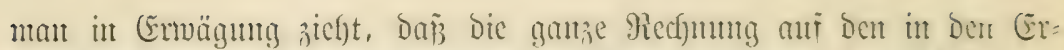

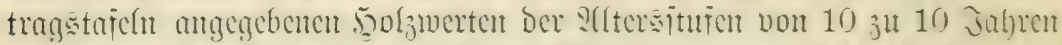

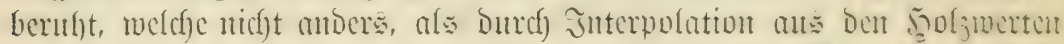

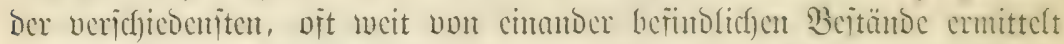

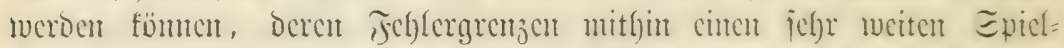

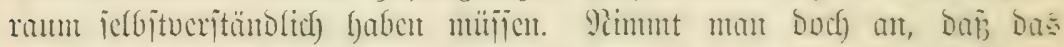
Ergeonis ber ?htsfluppicrumg cincs Bcitanocs als gut ju bejeiduncu ict.

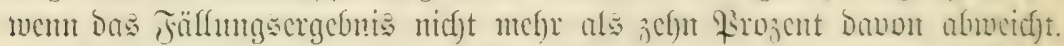

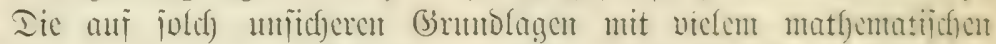

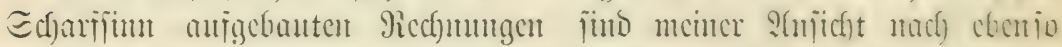

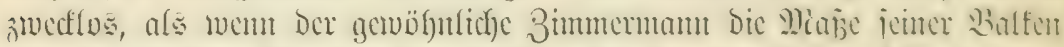

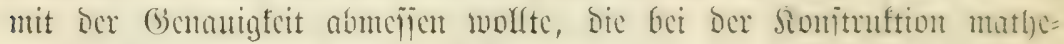

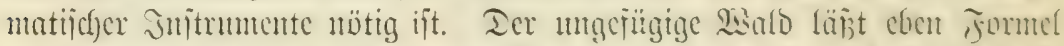

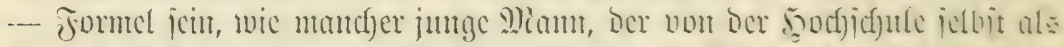

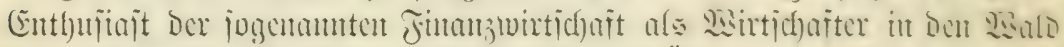

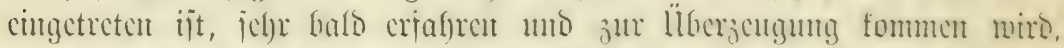

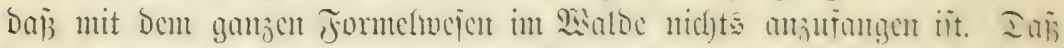

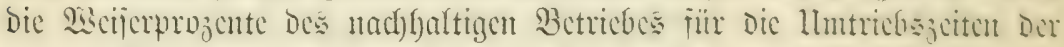

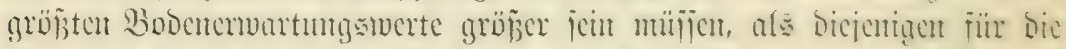

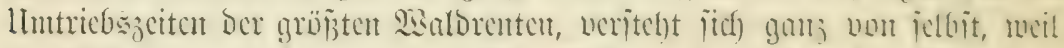

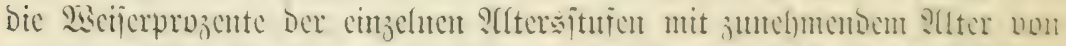

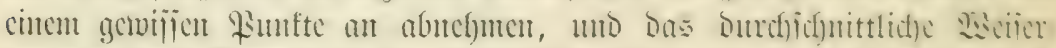

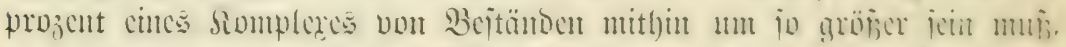

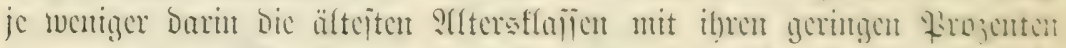
vertreten futb. Wer baber barmif ansgeht, ans bes in jemem math.

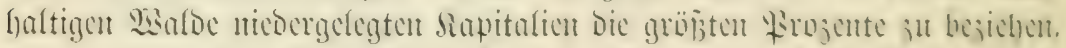

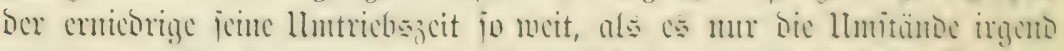

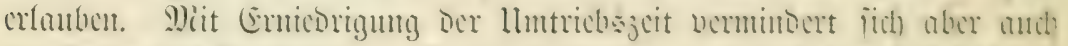

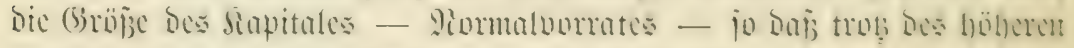

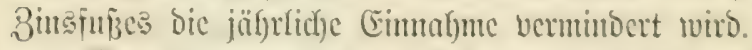

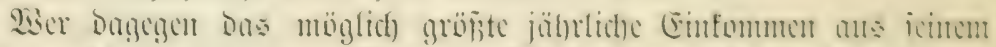

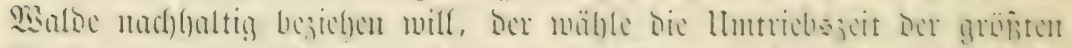

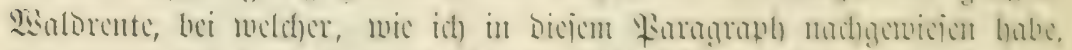

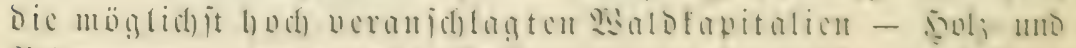

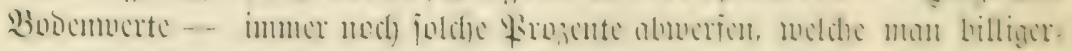
uccife num verlangen fumt. 


\section{$\$ 17$.}

\section{Beijerprozente bes undjultigen Betricles utter Qfutucubuth

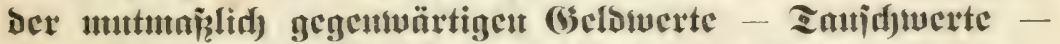

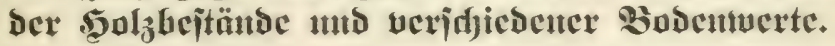

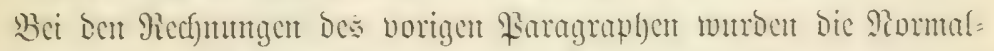
vorräte mit ifren (S) corauth

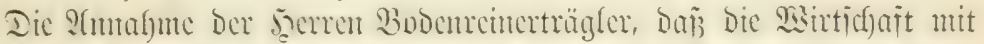

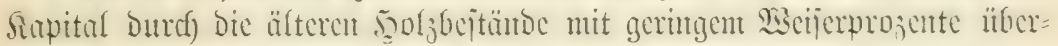

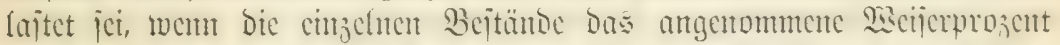

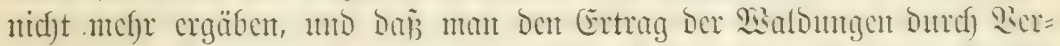

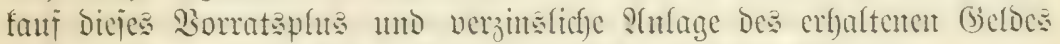
itcigen föme, ijt in iffere alfgemeinen fajijung irrtïmlich),

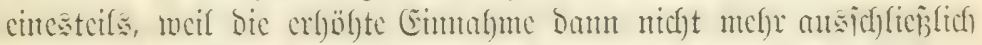

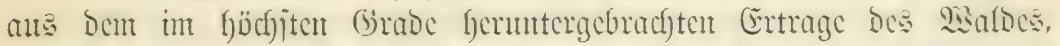
jonbern jugletef) aus Dem Ertringe cutes nebent)e laufenden Gelofaptals bejteft, umb

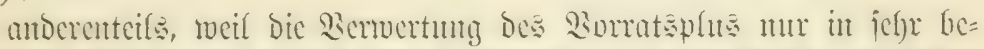

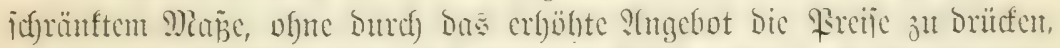

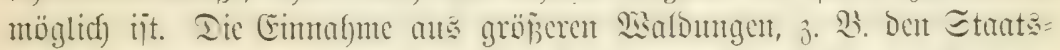

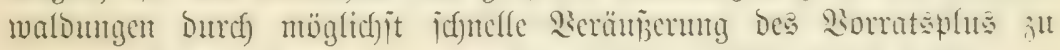

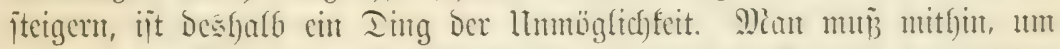

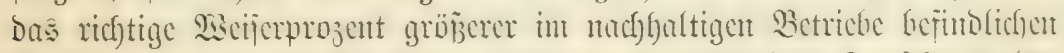

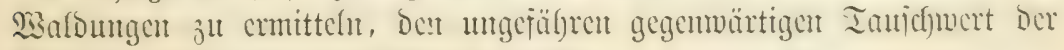
Diaterialvorräte zut veramichlagen juchent.

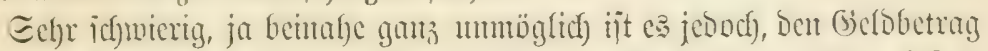

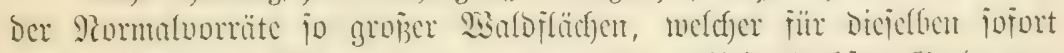

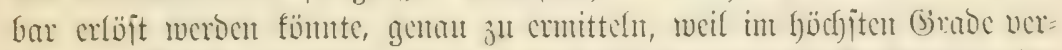

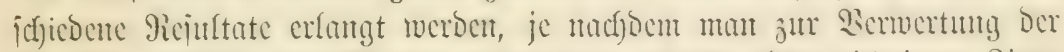

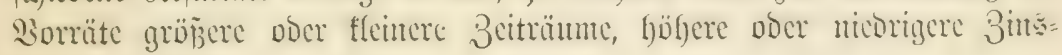
jū̇e unterjtellt.

Je nicbriger ber 3imsifm und je fïn

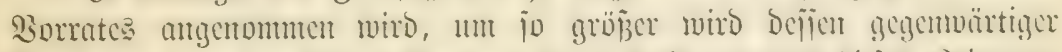

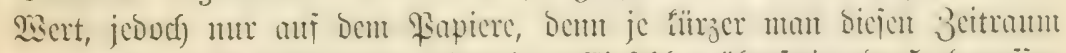

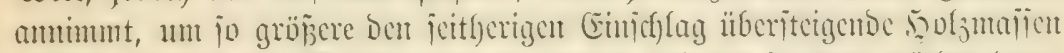

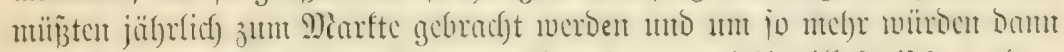

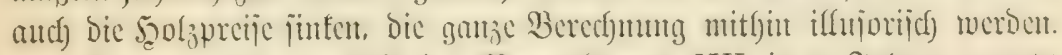

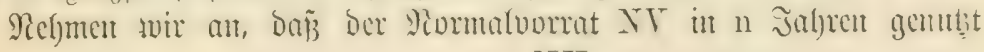
werben folle, jo wïrde bie Jafresqunote $\frac{N V}{n}$ betragen. 


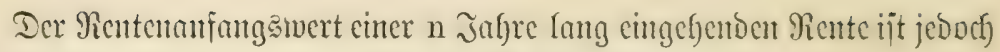

$$
=\mathrm{r} \times \frac{1,0 \mathrm{p}^{\mathrm{n}}-1}{1,0 \mathrm{p}^{\mathrm{n}} \cdot 0,0 \mathrm{p}}
$$

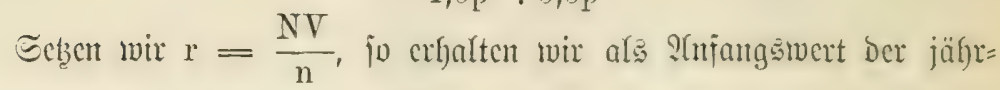

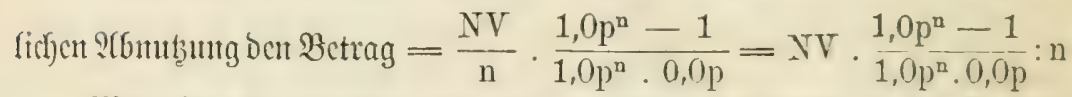

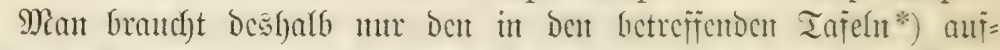

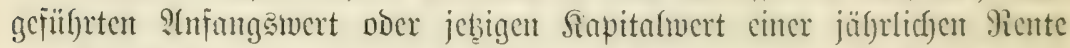

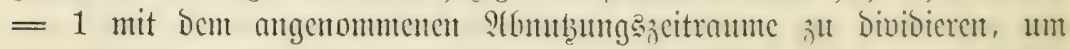
Den Faftor âu finden, mit welchem man ben 9iormalvorrat ju multi= plizieren hat, um Defjen gegenwärtigen Sapitalwert bei llnteritellumg cuner

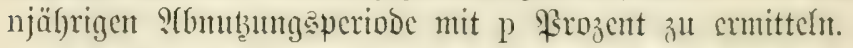

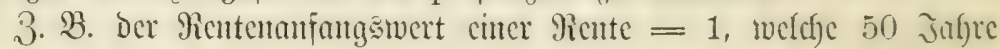

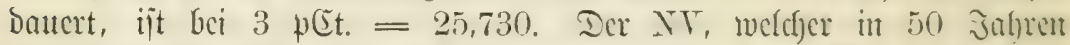

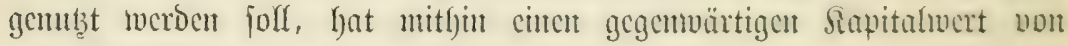
$\mathrm{NV} \cdot \frac{25,730}{50}=\mathrm{NV} \cdot 0,515$.

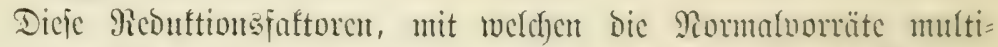

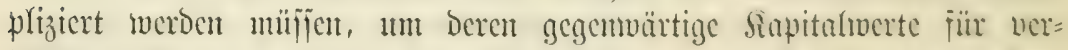

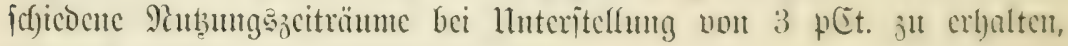
babe idf nadjitelyend zujanmengeitelft.

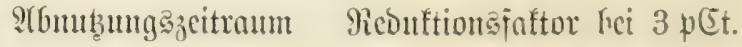

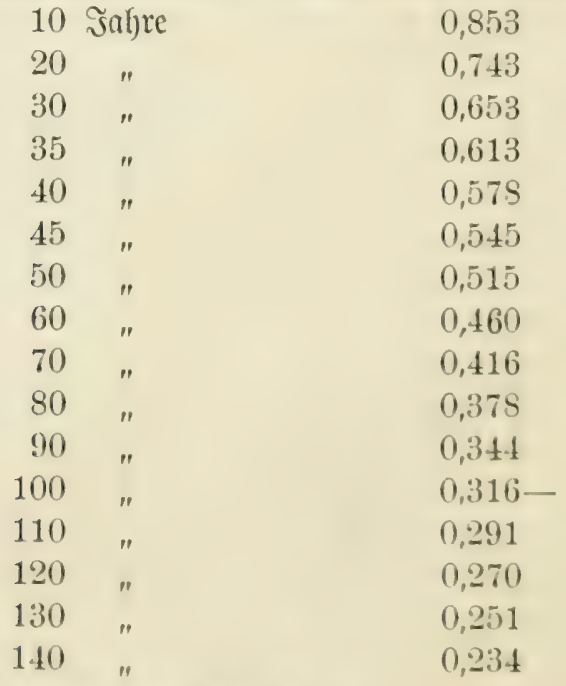

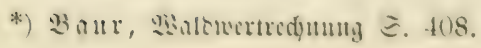




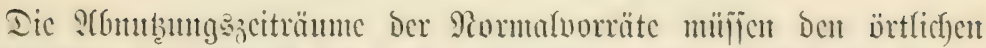

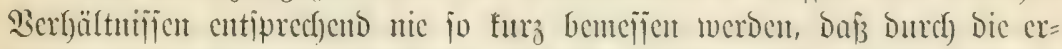

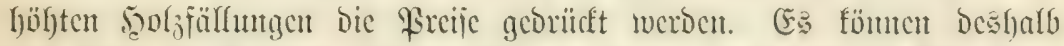

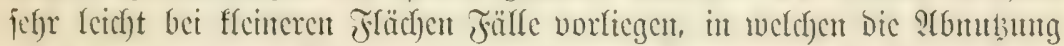

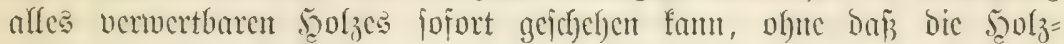

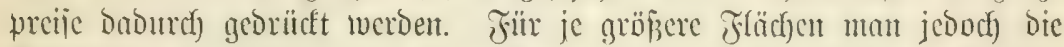

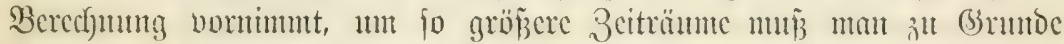
legen. Bei Dem regehnäßigen (S)ange Der Mittichaft wiro befauttlich, ber

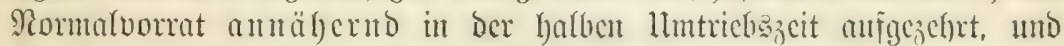

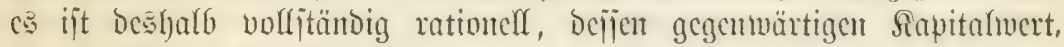

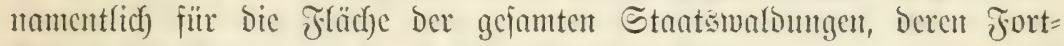

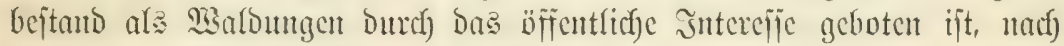
Den Reouftiontafaftoren für $\frac{\mathrm{u}}{2}$ zu berect)nen.

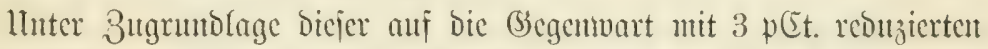
Sapitalwerte Der Rormaloorräte in Den Epalten $g$ habe ich) Dic Mirt=

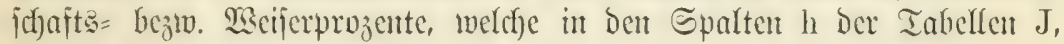
$K$ uno $L$ eingetragen fino, berechnet.

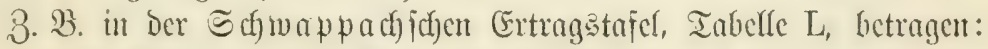

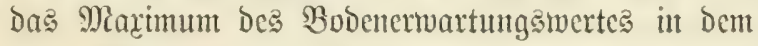

SIter vort 10 Jahren . . . . . . 1598,0 Ill

Der reduzicrte Normalvorrat fïr bic limtriehszeit ber

größsten $\mathfrak{B a l o r e n t e ~}(\mathrm{u}=120)$

Summe $\frac{2087,6}{3685,6}$

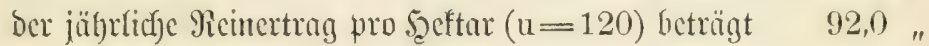

$$
\text { Daber Weiferprozent }=\frac{92,0 \times 100}{3685,6}=2,50 \text { " }
$$

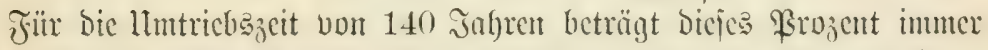
nod) 2,38 p(Et. mitfjin jo vicl, ale man bitligernveife mur verlangen fann. (5e geft ficraus bcrvor, wic vollftündig gerechtfertigt, aud) vom finan= zielfen Standpunfte a 3 , bie friffer mitgeteilte ?(nfid)t $\Xi$ d)wappad)

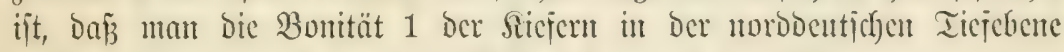
in cince Ilntricbsjeit von 140 Jal)ren bewirtichaften folle.

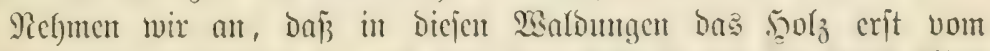

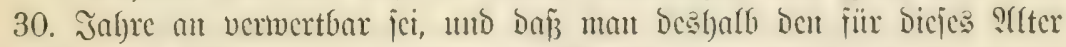

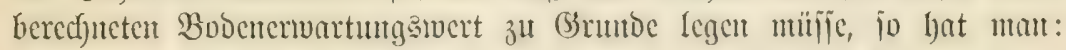

Be fïr $(\mathrm{u}=30)^{\circ}$. . . . . 1263,0 $/ \mathrm{l}$

Reouzierter NV für $(u=120) \cdot \frac{2087,6}{\text { Summe }}=\frac{3350,6 \text { Ill }}{3}$

Dafer $\mathfrak{W e i j e r p r o z e n t}=\frac{92 \times 100}{3350,6}=2,75$ " 
Iic mit Den berichicoenen Bobenverten mo ben auj bie biegenwart

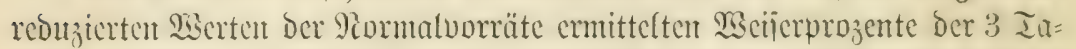

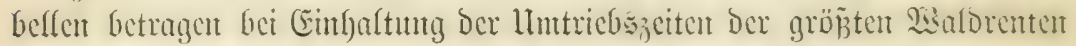
fïr bie Ertragstafel von Sudeid) . . 5,62-7,15 p(et.

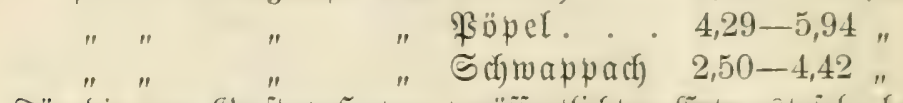

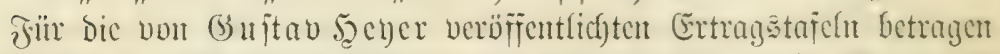
bic mit Den 3 verffficbenten Bobenverten unto mit ben anf Die Begenmart reduzierten Merten Der Pormalvorräte berechueten Meijerprojente bei Der IImtriebazzeit Der grȫ̄ten Warbrenten

$$
\begin{aligned}
& \text { für bic Buche . . . . . . . . 4,47-5,19 p@t. } \\
& \text { " "Fichte . . . . . . 3,85-5,77 " } \\
& \text { " " Sicier . . . . . 4, 4, 28-6,30 " }
\end{aligned}
$$

Man vergleidge bic Tabelfe $C$ in Baurs Centralblatt vom :Gugut 1888, ङ. 444, weldye ich nachitefyent wiedergebe.

$$
\text { (Siefe bic Tabelle C auf } \text { S. 61.) }
$$

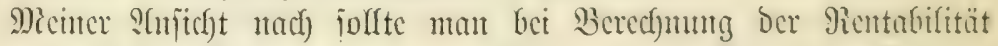

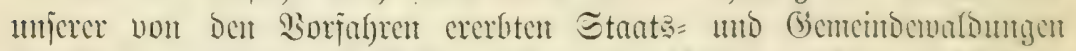

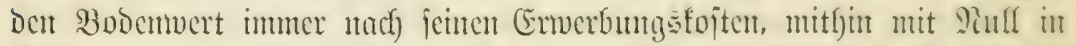
?hjat, bringen. Bir erhalten Dam bei unjeren 3 Iafeln I, II uno III

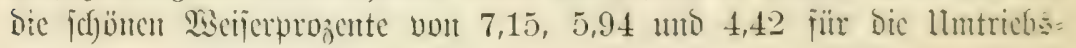
zeiten ber größ̄̈ten Malbrenten.

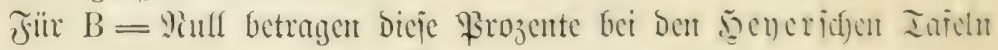
bei ber Budfe $=5,19$,

$$
\begin{aligned}
& " \text { " Fichte }=5,77, \\
& " \text { Sicfer }=6,33 .
\end{aligned}
$$

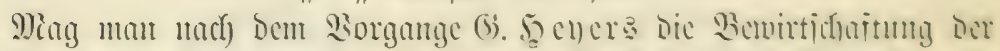

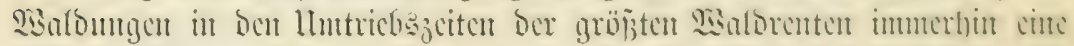

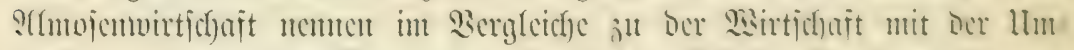

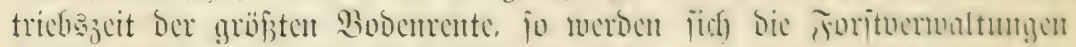

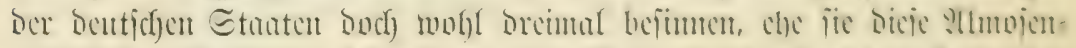

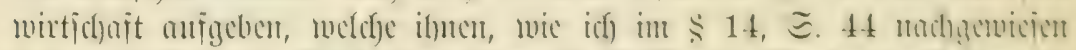

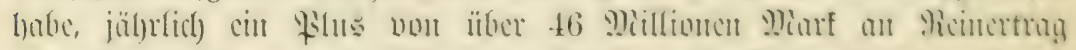

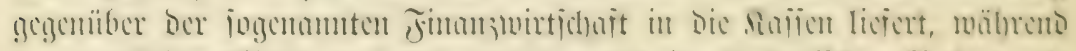

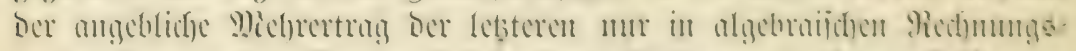

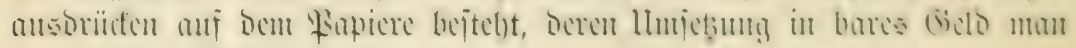
vergeblich verjudyen wiinto.

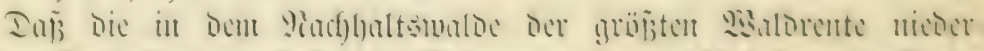

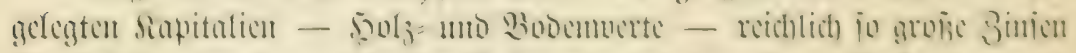

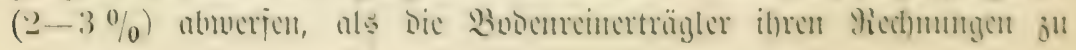




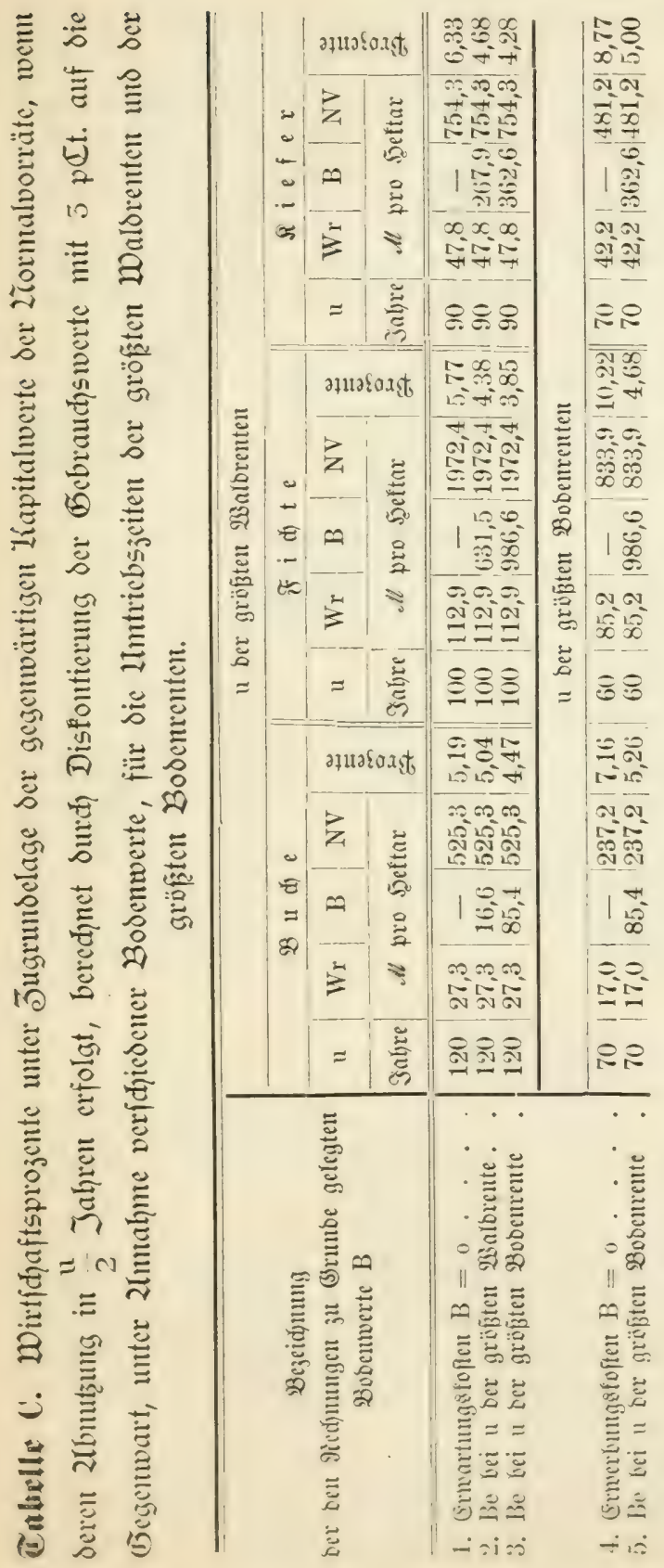

Sirumbe legen mitijen, went fie nicht triebszzeten gelangen wollen, bei rucleyen bic srochtwalomirtichajt aufigiten mur, glautbo ich) volljtänbig uncf)ge= woicien ju babent.

Dic Braftifer, weldye utidyt gleidy ben reincu Ifyedetifern ben givald mur Dutret) bic Brifle iffer algebrai= jclyen Formeln betrach)= tet faben, jefent and jel)r gुut cin, Daj nad) Den bis jeţt worlicgen= Den Erfalynutgen dic lintrichisciten DCr gröpisten Bodenrenten vid jult nitcorig fimb, ın cine juectmǟĩge Sodymaldowirtichait mit Denjelben betreibent j̆lt föıment.

2amentlick iit firait aud bicjer ?(ut= jidft. Ierjelfe fintot jedocly ben (situto bie= ier nicorigen llmtricbs: zeitcu nitht itt $\mathrm{DCm}$ Primipe Dex Buodent= reincrtragaleflec, jout bern it ber Ricmed

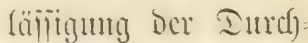
foritumęcu, moranj icf fichut in ?ionember: beite des Ecutralblat

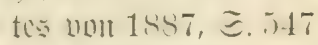
fjumgetvicjen lgabe. 


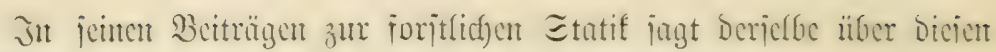
ßunft:

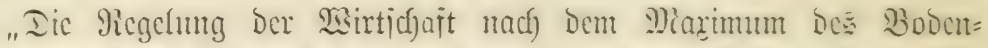

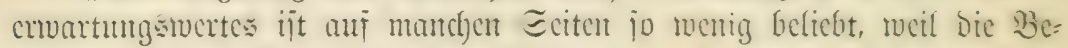

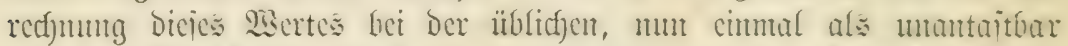

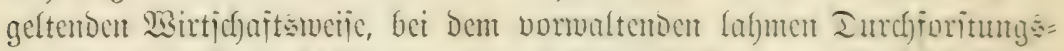

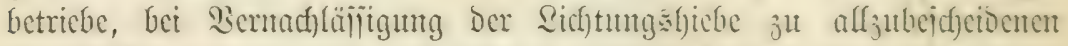

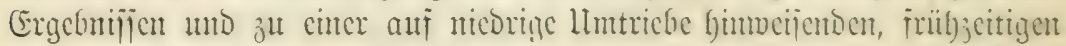
Sulfmination füffrt."

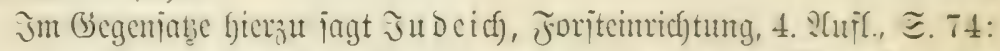

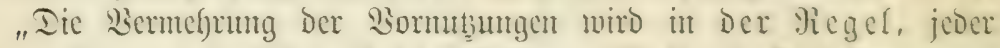

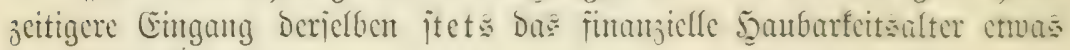
Geraboritcen."

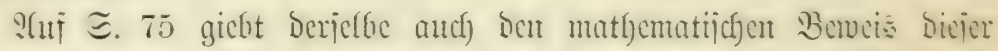

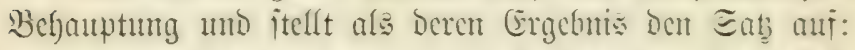

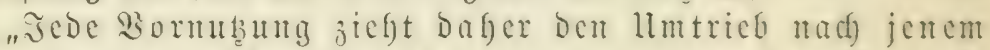
selter bin, in weldent ie erjolgt, je niedriger ber llmtrieb, Dejto öfter fehrt jie wieber."

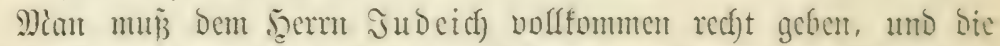

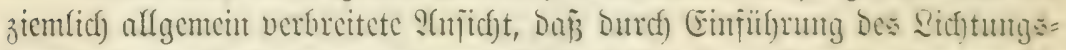

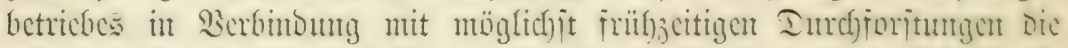

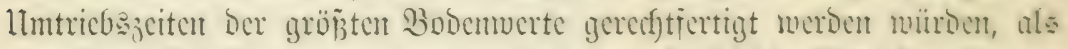

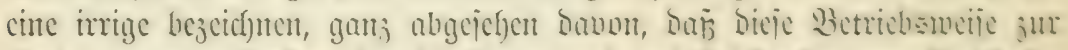

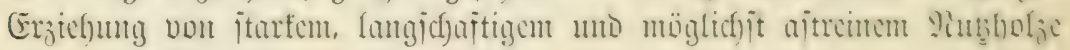
untruglich) ijt.

\section{$\S 18$.}

\section{Exjluiflucuerfung.}

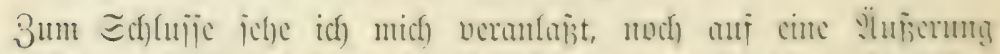

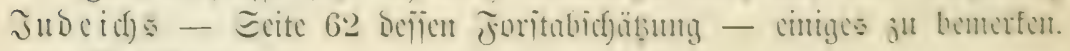

Derictbe jagt:

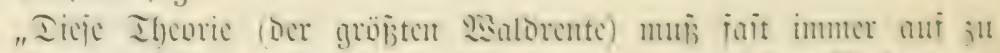

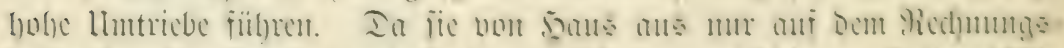

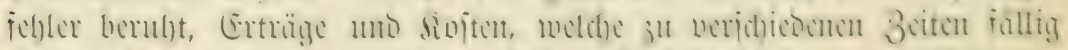

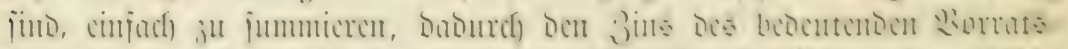

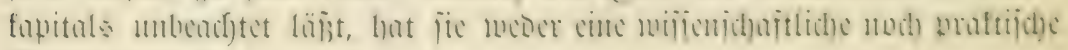

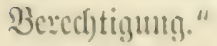

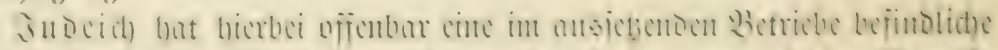

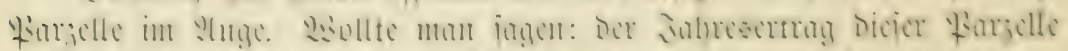

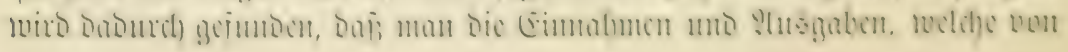




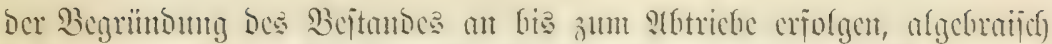

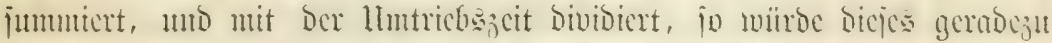

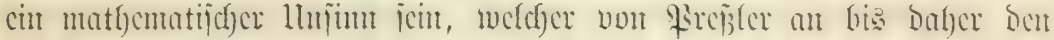

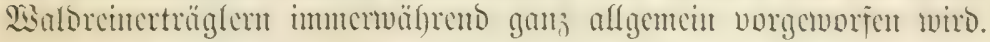

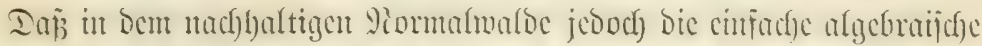

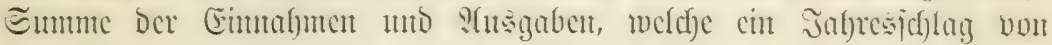

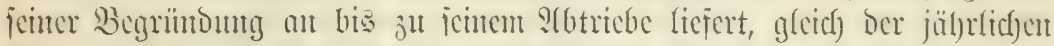

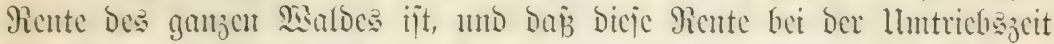

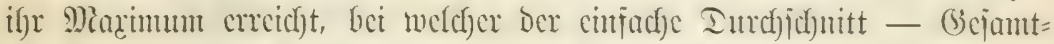

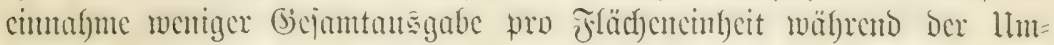

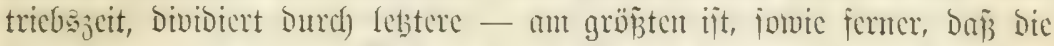

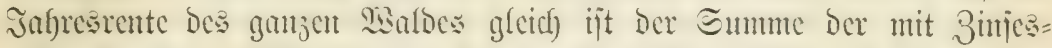

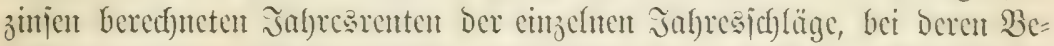

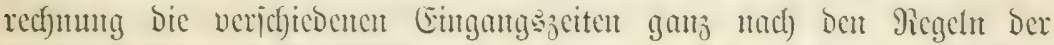

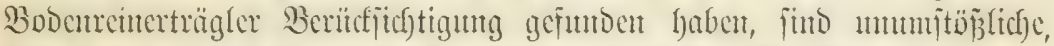

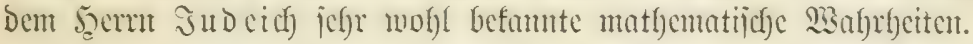

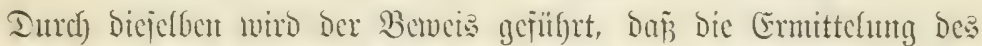
cinfacfan Durdfichuttesertrages bei Den Pormalwalde gerube jo gut auf

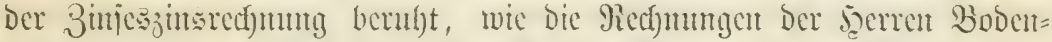

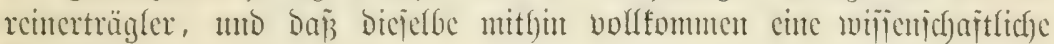
Berechtigutg bejtỉt.

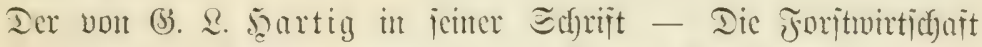
nac) if)rem ganzen limjantge in gebrängter Sïrze. Ricutlingen 1832 -

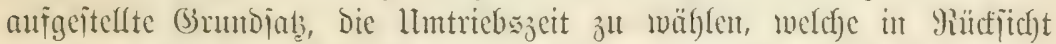

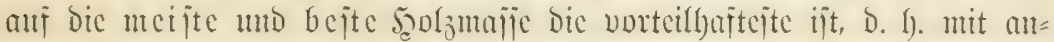

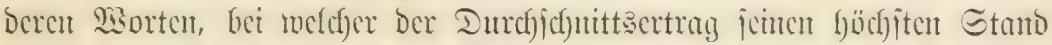

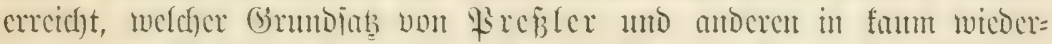

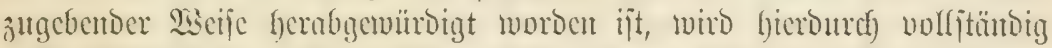

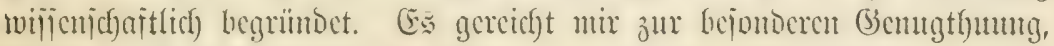
dieję zuerit nadjgetwiejen åu haben.

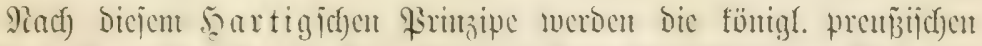

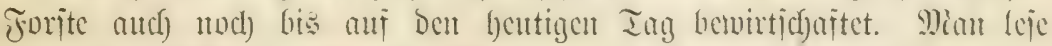

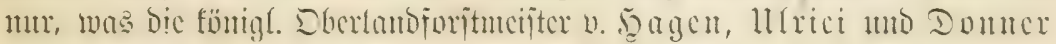
Dariber gejdrieben baben.

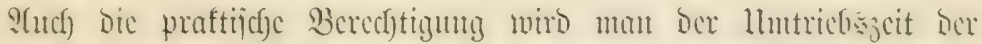

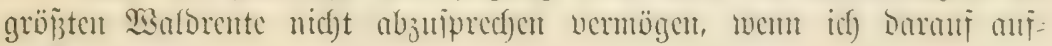
merfiam madje:

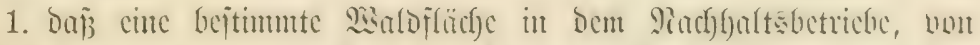

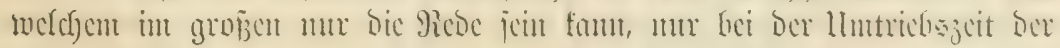




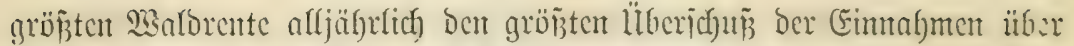

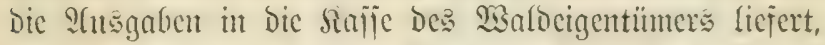

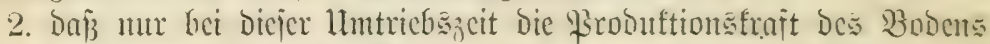
volfitändig atsgenußgt werben fam, und

3. Dâj bemoch Dic in dem Walde nicocrgelegten Siapitalien - ñol umb Bobcumert - jich jo hod) verginjen, als mant billigerwecije mur ver= langen fant.

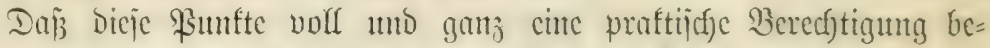

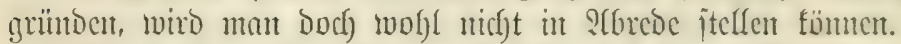

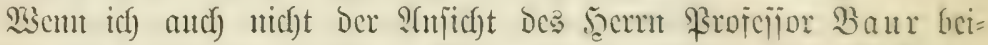

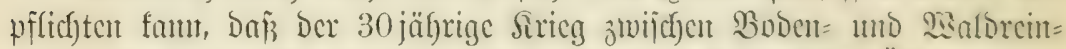

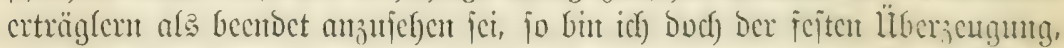
Daż anjtalten in Berbinbung mit genaucu jtatijtijdyen Ermittefungen ïfer

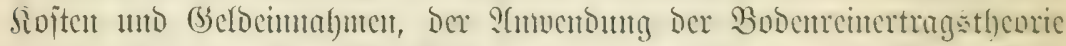

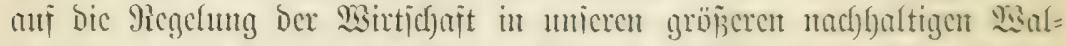

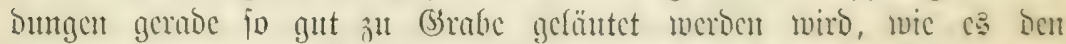

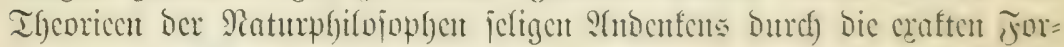

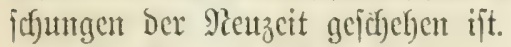

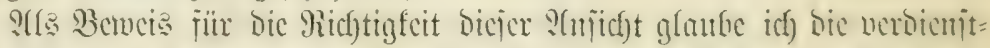

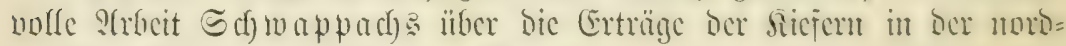
Deutjodjen Ticjebene anfül)ren z̆ fömen. 


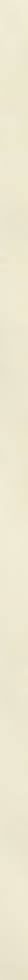





\section{LIBRARY}

UNIVERSITY OF TOPONTO

SD

393

B67

BioMed
Bose, Heinrich Ludwig

Das forstliche Weiserprozent

PLEASE DO NOT REMOVE

CARDS OR SLIPS FROM THIS POCKET

UNIVERSITY OF TORONTO LIBRARY

$[84991]$ 
